\title{
Adding complex fermions to the Grassmannian-like coset model
}

\author{
Changhyun Ahn ${ }^{\mathrm{a}}$ \\ Department of Physics, Kyungpook National University, Daegu 41566, Korea
}

Received: 21 July 2021 / Accepted: 21 November 2021 / Published online: 22 December 2021

(C) The Author(s) 2021

\begin{abstract}
In the $\mathcal{N}=2$ supersymmetric coset model, $\frac{S U(N+M)_{k} \times S O(2 N M)_{1}}{N)_{k+M} \times U(1)_{N M(N+M)(k+N+M)}}$, we construct the $S U(M)$ nonsinglet $\mathcal{N}=2$ multiplet of spins $\left(1, \frac{3}{2}, \frac{3}{2}, 2\right)$ in terms of coset fields. The next $S U(M)$ singlet and nonsinglet $\mathcal{N}=2$ multiplets of spins $\left(2, \frac{5}{2}, \frac{5}{2}, 3\right)$ are determined by applying the $\mathcal{N}=2$ supersymmetry currents of spin $\frac{3}{2}$ to the bosonic singlet and nonsinglet currents of spin 3 in the bosonic coset model. We also obtain the operator product expansions (OPEs) between the currents of the $\mathcal{N}=2$ superconformal algebra and above three kinds of $\mathcal{N}=2$ multiplets. These currents in two dimensions play the role of the asymptotic symmetry, as the generators of $\mathcal{N}=2$ "rectangular $W$-algebra", of the $M \times M$ matrix generalization of $\mathcal{N}=2 A d S_{3}$ higher spin theory in the bulk. The structure constants in the right hand sides of these OPEs are dependent on the three parameters $k, N$ and $M$ explicitly. Moreover, the OPEs between $S U(M)$ nonsinglet $\mathcal{N}=2$ multiplet of spins $\left(1, \frac{3}{2}, \frac{3}{2}, 2\right)$ and itself are analyzed in detail. The complete OPE between the lowest component of the $S U(M)$ singlet $\mathcal{N}=2$ multiplet of spins $\left(2, \frac{5}{2}, \frac{5}{2}, 3\right)$ and itself is described. In particular, when $M=2$, it is known that the above $\mathcal{N}=2$ supersymmetric coset model provides the realization of the extension of the large $\mathcal{N}=4$ nonlinear superconformal algebra. We determine the currents of the large $\mathcal{N}=4$ nonlinear superconformal algebra and the higher spin- $\frac{3}{2}, 2$ currents of the lowest $\mathcal{N}=4$ multiplet for generic $k$ and $N$ in terms of the coset fields. For the remaining higher spin- $\frac{5}{2}, 3$ currents of the lowest $\mathcal{N}=4$ multiplet, some of the results are given.
\end{abstract}

\section{Contents}

1 Introduction . . . . . . . . . . . . . . . 2

2 Review ................. 4

2.1 The singlet currents of spins $\left(1, \frac{3}{2}, \frac{3}{2}, 2\right) \ldots \ldots 4$

2.1.1 The role of complex fermions ..... . 4

a e-mail: ahn@knu.ac.kr (corresponding author)
2.1.2 The $\mathcal{N}=2$ superconformal algebra . . . 5

2.1.3 The so far known currents . . . . . . . 6

2.2 The nonsinglet currents of spins $\left(1, \frac{3}{2}, \frac{3}{2}, 2\right)$. . 6

2.3 The regular condition .......... . 6

3 The nonsinglet multiplet of spins $\left(1, \frac{3}{2}, \frac{3}{2}, 2\right) \ldots 7$

3.1 The spin-2 current . . . . . . . . . . 7

3.2 The OPEs with the currents of $\mathcal{N}=2$ superconformal algebra . . . . . . . . . . . 7

3.3 Some OPEs with the spin-2 current $K^{a}$. . . 9

4 The singlet multiplet of spins $\left(2, \frac{5}{2}, \frac{5}{2}, 3\right) \ldots 10$

4.1 Construction of lowest component . . . . . . . 10

4.2 Construction of second and third components . 12

4.3 Construction of last component . . . . . . . . . 12

4.4 The OPEs with the currents of $\mathcal{N}=2$ superconformal algebra . . . . . . . . . . . 13

5 The nonsinglet multiplet of spins $\left(2, \frac{5}{2}, \frac{5}{2}, 3\right) \ldots 15$

5.1 Construction of lowest component . . . . . 15

5.2 Construction of second and third components . 16

5.3 Construction of last component . . . . . . . . 17

5.4 The OPEs with the currents of $\mathcal{N}=2$ superconformal algebra . . . . . . . . . . . 18

6 The OPEs between the nonsinglet multiplet of spins $\left(1, \frac{3}{2}, \frac{3}{2}, 2\right)$ and itself . . . . . . . . . . 20

6.1 The OPEs with lowest component . . . . . . 20

6.2 The OPEs with the second component . . . . . 21

6.2.1 The OPE with spin-2 current . . . . . . 22

6.3 The OPEs with the third component . . . . . 23

6.3.1 The OPE with the spin-2 current . . . . . 24

6.4 The final OPE . . . . . . . . . . . 24

7 Towards the OPEs between the singlet multiplet of spins $\left(2, \frac{5}{2}, \frac{5}{2}, 3\right)$ and itself . . . . . . . . 25

8 The extension of the large $\mathcal{N}=4$ nonlinear superconformal algebra for $M=2$. . . . . . . . . 26

8.1 Four spin- $\frac{3}{2}$ currents . . . . . . . . . . 26

8.2 Higher spin- $\frac{3}{2}$ currents . . . . . . . . . . 26

8.3 Higher spin-2 currents . . . . . . . . . . . . 27

8.4 Higher spin- $\frac{5}{2}, 3$ currents . . . . . . . . . . 27

8.5 For $M=3 \ldots \ldots \ldots$. . . . . . . . . 28 
9 Conclusions and outlook . . . . . . . . . 28

A The $\mathcal{N}=2$ superconformal algebra with a modified stress energy tensor . . . . . . . . . . . . . . 29

A.1 The OPEs between the spin- $\frac{1}{2}$ operators and the spin-1 operators . . . . . . . . . . . 29

A.2 The OPEs between the spin-1 operators . . . . 30

A.3 The OPEs between the spin- $\frac{1}{2}$ operators and the currents of $\mathcal{N}=2$ superconformal algebra . . 30

A.4 The OPEs between the spin-1 operators and the currents of $\mathcal{N}=2$ superconformal algebra . . . 30

A.5 The $\mathcal{N}=2$ superconformal algebra . . . . . 31

B The $\mathcal{N}=2$ primary conditions . . . . . . . . . 32

C Some OPEs between the spin-1 currents and the singlet and nonsinglet spin-3 currents in Sects. 4 and 5

..................... 32

C.1 The OPE between $J^{(\rho \bar{i})}(z) W^{(3)}(w)$. . . . . . 32

C. 2 The OPE between $J^{(\bar{\sigma} j)}(z) W^{(3)}(w)$. . . . . 33

C.3 The OPE between $J^{(\rho \bar{i})}(z) P^{a}(w) \ldots . . . .34$

C.4 The OPE between $J^{(\bar{\sigma} j)}(z) P^{a}(w) \ldots . . . .35$

D Some OPEs in terms of coset fields in Sect. 6 . . . 36

E The OPEs between the nonsinglet spin-2 operators in Sect. 6 . . . . . . . . . . . . . 37

$\mathrm{F}$ The OPEs between the singlet spin-2 operators in Sect. 7 . . . . . . . . . . . . 40

G Some of the defining relations in Sect. 8 . . . . . 41

$\mathrm{H}$ The higher spin- $\frac{5}{2}$ currents for fixed $N=5$ and $M=2$ in Sect. 8 . . . . . . . . . . . . 42

References................ . 44

\section{Introduction}

It is known that the most general coset in the bosonic theory can be described by [1]

$\frac{S U(N+M)_{k}}{S U(N)_{k} \times U(1)_{N M(N+M) k}}$,

where the three parameters, $k, M$ and $N$ are present. ${ }^{1}$ According to the observation of [3], this coset model is dual to $M \times M$ matrix generalization of $A d S_{3}$ Vasiliev higher spin theory $[4,5]$ by taking the appropriate limit on these parameters. Note that for $M=1$ case, the Gaberdiel-Gopakumar conjecture [6] can be seen and see also [7-9] for the relevant works. At the particular value of the level $k$ with generic $N$ and $M$, the operator product expansion (OPE) between the charged spin-2 current and itself leads to the one of the "rectangular" $W$-algebra [10] with $S U(M)$ symmetry which is the asymptotic symmetry of $A d S_{3}$ higher spin theory [3]

1 The possibility of four parameters in the different coset model is studied in [2]. and see also [11]. For generic $k, N$ and $M$, this OPE is further studied in [12] and see also [13,14]. ${ }^{2}$

Why do we add the complex fermions into the above coset model (1.1)? This is one of the ways to make the bosonic theory to be the supersymmetric theory. In other words, by using the fermionic operators of spin- $\frac{1}{2}$, we can construct the half integer currents including the supersymmetry generators of spin- $\frac{3}{2}$ explicitly. ${ }^{3}$ We would like to discuss about the supersymmetric version of (1.1) and it is given by $[1,30]$

$\frac{S U(N+M)_{k} \times S O(2 N M)_{1}}{S U(N)_{k+M} \times U(1)_{N M(N+M)(k+N+M)}}$.

Note that there exists an $S O(2 N M)_{1}$ factor associated with complex fermions in the numerator and the levels in the denominator are changed appropriately. For $M=1$, by dividing (1.2) out the $S U(M=1)_{k+N}$ further, the $\mathcal{N}=2$ $A d S_{3}$ higher spin gravity is related to the Kazama-Suzuki model [31,32], according to [33]. See also the relevant works in [27,34-45]. For $M=2$, the above coset arises in the context of the large $\mathcal{N}=4$ holography [46]. See also previous works on this holography [2,44,47-71].

For generic $M>2$, in [30], the extension of $\mathcal{N}=2$ superconformal algbra is described. The generators of $\mathcal{N}=2$ superconformal algebra are given by the spin-1, $\frac{3}{2}, \frac{3}{2}, 2$ currents and denoted by $K, G^{+}, G^{-}, T$ respectively. ${ }^{4}$ The extra generators of $S U(M)$ nonsinglets are classified by the spin1 currents, spin- $\frac{3}{2}$ currents, and the spin- 2 currents. Their numbers are given by $\left(M^{2}-1\right), 2\left(M^{2}-1\right)$ and $\left(M^{2}-1\right)$ respectively. If we increase the spin by one, then there are the spin- 2 currents, spin- $\frac{5}{2}$ currents, and the spin- 3 currents of $S U(M)$ singlets and nonsinglets. Their numbers are given by $M^{2}, 2 M^{2}$ and $M^{2}$ respectively. As we increase further, then the general features of $S U(M)$ singlets and nonsinglets go through: there are the $M^{2}$ spin- $s$ currents, the $2 M^{2}$ spin-

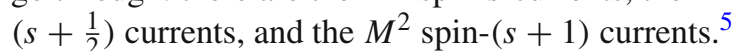

We present them here, in the notation of $\mathcal{N}=2$ multiplet, ${ }^{6}$ with the assignment of spin and the $S U(M)$ index

\footnotetext{
2 There is a previous work on the Grassmannian coset model in [15].

3 Without introducing the fermions, we can have the supersymmetric version of (1.1) by considering the special value of the level $k$. This is because we can realize the various spin- 1 currents in terms of fermions. For example, see also [16-29].

4 The OPE between $G^{+}$and $G^{-}$does not produce the standard $\mathcal{N}=2$ superconformal algebra for generic $M>1$ because the first order pole of this OPE has the additional terms. However, we are using the same terminology " $\mathcal{N}=2$ superconformal algebra" in this paper

5 The $\mathcal{N}=2$ "rectangular" $W$-algebra appears as the asymptotic symmetry of the $M \times M$ matrix generalization of $\mathcal{N}=2 A d S_{3}$ higher spin theory [3].

${ }^{6}$ Strictly speaking, when we are saying about " $\mathcal{N}=2$ multiplet" in this paper, the four component currents do not satisfy the standard $\mathcal{N}=2$ primary conditions with the currents $\left(K, G^{+}, G^{-}, T\right)$ : there are some higher order poles appear and some structure constants appear differently.
} 
$a=1,2, \ldots,\left(M^{2}-1\right)$ as follows:

$$
\begin{aligned}
& \left(W^{-(1), 0}, G^{+\left(\frac{3}{2}\right), 0}, G^{-\left(\frac{3}{2}\right), 0}, W^{+(2), 0}\right) \\
& \quad \equiv\left(K, G^{+}, G^{-}, T\right), \\
& \left(W^{-(1), a}, G^{+\left(\frac{3}{2}\right), a}, G^{-\left(\frac{3}{2}\right), a}, W^{+(2), a}\right), \\
& \left(W^{-(2), 0}, G^{+\left(\frac{5}{2}\right), 0}, G^{-\left(\frac{5}{2}\right), 0}, W^{+(3), 0}\right), \\
& \left(W^{-(2), a}, G^{+\left(\frac{5}{2}\right), a}, G^{-\left(\frac{5}{2}\right), a}, W^{+(3), a}\right), \\
& \left(W^{-(3), 0}, G^{+\left(\frac{7}{2}\right), 0}, G^{-\left(\frac{7}{2}\right), 0}, W^{+(4), 0}\right), \\
& \left(W^{-(3), a}, G^{+\left(\frac{7}{2}\right), a}, G^{-\left(\frac{7}{2}\right), a}, W^{+(4), a}\right), \\
& \left(W^{-(s), 0}, G^{+\left(s+\frac{1}{2}\right), 0}, G^{-\left(s+\frac{1}{2}\right), 0}, W^{+(s+1), 0}\right), \\
& \left(W^{-(s), a}, G^{+\left(s+\frac{1}{2}\right), a}, G^{-\left(s+\frac{1}{2}\right), a}, W^{+(s+1), a}\right), \quad \cdots
\end{aligned}
$$

In (1.3), the generators of $\mathcal{N}=2$ superconformal algebra are denoted by $\left(K, G^{+}, G^{-}, T\right)$. Each current of half integer spins appears in the same $\mathcal{N}=2$ multiplet while each current of integer spins appears in the different $\mathcal{N}=2$ multiplets. The coset realization on the above currents is done for the generators of $\left(K, G^{+}, G^{-}, T\right)$ and the next three kinds of $\left(W^{-(1), a}, G^{+\left(\frac{3}{2}\right), a}, G^{-\left(\frac{3}{2}\right), a}\right)$ so far in [30]. ${ }^{7}$

In this paper, we would like to construct the coset realization for the following currents

$$
\begin{aligned}
& \text { (known, known, known, } \left.W^{+(2), a}\right), \\
& \left(W^{-(2), 0}, G^{+\left(\frac{5}{2}\right), 0}, G^{-\left(\frac{5}{2}\right), 0}, W^{+(3), 0}\right), \\
& \left(W^{-(2), a}, G^{+\left(\frac{5}{2}\right), a}, G^{-\left(\frac{5}{2}\right), a}, W^{+(3), a}\right) .
\end{aligned}
$$

Here, the first three components of the first $\mathcal{N}=2$ multiplet (1.4) are known in terms of coset fields.

Moreover, we describe some of the OPEs between the currents for low spins. Then how we can construct the currents in (1.4) in terms of coset fields explicitly? For the spin-2 current appearing in the first line of (1.4), we can use both the previous known currents which belong to the same $\mathcal{N}=2$ multiplet and the generators of $\left(K, G^{+}, G^{-}, T\right)$. For the currents belonging to the second and third $\mathcal{N}=2$ multiplets in (1.4), we can use the previous known currents of spin-2, 3 currents in the bosonic coset model (1.1). We figure out that the singlet spin-3 current $W^{(3)}$ will appear in the last component of the second $\mathcal{N}=2$ multiplet while the nonsinglet spin-3 current $P^{a}$ will appear in the last component of the third $\mathcal{N}=2$ multiplet and the nonsinglet spin-2 current $K^{a}$

\footnotetext{
$\overline{7}$ We are using the notation [30] of $\mathcal{N}=2$ "rectangular" $W$-algebra in the bulk for its dual CFT currents in (1.3).
}

will appear in the first component of the third $\mathcal{N}=2$ multiplet, as the $\mathcal{N}=2$ supersymmetric versions. This is because the OPE between the spin- 2 current $K^{a}$ and the spin- 2 current $K^{b}$ leads to the spin-3 current $P^{c}$ in the bosonic coset model. Furthermore, when we fix $M=1$, the singlet spin3 current arises in the last component of the corresponding $\mathcal{N}=2$ multiplet (For example, [27,37,39]) and therefore it is natural to think about the above description for general $M$ case.

One way to determine the $\mathcal{N}=2$ superpartners of the spin-2, 3 currents found in the bosonic coset model is to consider, as a first step, the OPEs between the $\mathcal{N}=2$ supersymmetry generators $G^{ \pm}$and the spin-3 currents $W^{(3)}$ and $P^{a}$ of the bosonic theory.

Then we will obtain the intermediate spin- $\frac{5}{2}$ currents in the specific poles which depend on the complex fermions as well as the bosonic currents. Further computations for the OPEs between the above supersymmetry generators $G^{ \pm}$and these intermediate spin- $\frac{5}{2}$ currents obtained at the previous stage can be performed. Then we will obtain the spin- 2 currents which will contain both the complex fermions and the bosonic currents as before. Then we have singlet spin-2 current $W^{-(2), 0}$ and the nonsinglet spin-2 currents $W^{-(2), a}$ as in (1.4). By calculating the OPEs between the spin- $\frac{3}{2}$ currents $G^{ \pm}$and these spin-2 currents obtained newly, we can determine the singlet spin- $\frac{5}{2}$ currents $W^{ \pm\left(\frac{5}{2}\right), 0}$ and the nonsinglet spin- $\frac{5}{2}$ currents $W^{ \pm\left(\frac{5}{2}\right), a}$. In general, they are different from the above intermediate spin- $\frac{5}{2}$ currents. Finally, after further action of supersymmetry generators $G^{ \pm}$on these spin- $\frac{5}{2}$ currents, the singlet spin-3 current $W^{+(3), 0}$ and the nonsinglet spin-3 currents $W^{+(3), a}$ can be determined completely.

So far, the OPEs we are considering are the ones between the currents of the $\mathcal{N}=2$ superconformal algebra (the first line of (1.3)) and the currents of the singlet and nonsinglet $\mathcal{N}=2$ multiplets (1.4). Therefore, the right hand sides of these OPEs look similar to the behavior of standard $\mathcal{N}=2$ primary currents. The difference is that we observe that there appear some other additional singular terms and other type of currents living in other $\mathcal{N}=2$ multiplet.

The next things we should do, for the simplest cases, is to compute (1) the OPEs between the currents in the first $\mathcal{N}=2$ multiplet in (1.4) and (2) the OPE between $W^{-(2), 0}$ and itself.

In this case we do expect that the right hand sides of these OPEs will produce other $\mathcal{N}=2$ multiplets nontrivially. For the OPEs between the first nonsinglet $\mathcal{N}=2$ multiplet in (1.4), there exist new primary spin- $\frac{5}{2}$ currents having two free indices and primary spin-3 current also having two free 
indices. For the OPE between the lowest component and itself of the second singlet $\mathcal{N}=2$ multiplet in (1.4), there is no new primary current. Although we consider the OPE between the singlet spin-2 current and itself, we do expect that the OPEs between the currents of the singlet $\mathcal{N}=2$ multiplets $\left(W^{-(s), 0}, G^{+\left(s+\frac{1}{2}\right), 0}, G^{-\left(s+\frac{1}{2}\right), 0}, W^{+(s+1), 0}\right)$ do not contain the currents from the nonsinglet $\mathcal{N}=2$ multiplets, in the presence of the currents of the $\mathcal{N}=2$ superconformal algebra with modified stress energy tensor.

Furthermore, we can study the extension of the large $\mathcal{N}=$ 4 nonlinear superconformal algebra in the present context because we have some information on the coset fields via (1.4). The question is how to reorganize the currents of $\mathcal{N}=4$ multiplets in terms of the currents of $\mathcal{N}=2$ multiplets and the coset fields.

In this case, the parameter $M$ is fixed by 2 . We can try to obtain the relevant currents of the above extension by recalling the defining OPEs in [72] between them starting from the low spins. The nontrivial part is to obtain the currents of the the large $\mathcal{N}=4$ nonlinear superconformal algebra because the stress energy tensor appears very nontrivially (For example, [73]) and we should fix the normalizations for the spin-1 currents and the spin- $\frac{3}{2}$ currents correctly. Once we identify the currents of the the large $\mathcal{N}=4$ nonlinear superconformal algebra, then it is rather straightforward to determine the currents of the lowest $\mathcal{N}=4$ multiplet by using the defining relations in the OPEs in [72].

In Sect. 2, we review how we can add the complex fermions into the bosonic coset model and the coset realization for the $\mathcal{N}=2$ superconformal algebra is reviewed. We describe the known currents in (1.4). In Sect. 3, after finding the nonsinglet spin-2 currents $W^{+(2), a}$, we compute the OPEs between the first $\mathcal{N}=2$ multiplet in the second line of (1.3) and the $\mathcal{N}=2$ stress energy tensor in the first line of (1.3). In Sect. 4, by considering the OPEs between the spin- $\frac{3}{2}$ currents $G^{ \pm}$and the spin-3 current $W^{(3)}$ and analyzing the OPEs between the spin- $\frac{3}{2}$ currents $G^{ \pm}$and the spin- $\frac{5}{2}$ current found newly successively, the lowest current of the second $\mathcal{N}=2$ multiplet in the third of (1.3) can be obtained. Moreover, the remaining other three components of this $\mathcal{N}=2$ multiplet can be determined. As before, the OPEs between the second $\mathcal{N}=2$ multiplet in the third line of (1.3) and the $\mathcal{N}=2$ stress energy tensor in the first line of (1.3) are determined. In Sect. 5, we do this section by considering the third $\mathcal{N}=2$ multiplet in the fourth line of (1.3) by following the procedure done in Sect. 4. In Sect. 6, after analyzing the OPEs between the first $\mathcal{N}=2$ multiplet, the arising of the new primary currents of spin- $\frac{5}{2}, 3$ having the free indices $a b$ of $S U(M)$ is described. In Sect. 7, some of the OPEs between the second $\mathcal{N}=2$ multiplet are analyzed. In Sect. 8, for the
$M=2$ case, the coset realization gives us an extension of the large $\mathcal{N}=4$ nonlinear superconformal algebra [73-76] and some of the currents of the lowest $\mathcal{N}=4$ multiplet are given explicitly. In Sect. 9, we conclude our work and the future directions are given. The various Appendices are for the details in previous sections we describe.

\section{Review}

Some known results are reviewed in this section.

2.1 The singlet currents of spins $\left(1, \frac{3}{2}, \frac{3}{2}, 2\right)$

\subsubsection{The role of complex fermions}

In the coset

$$
\frac{S U(N+M)_{k} \times S O(2 N M)_{1}}{S U(N)_{k+M} \times U(1)_{N M(N+M)(k+N+M)}},
$$

the decomposition of $S U(N+M)$ into the $S U(N) \times S U(M)$ in the numerator can be performed as in the bosonic case [3]. We use the generators $\left(t^{\alpha}, t^{a}, t^{u(1)}, t^{(\rho \bar{i})}, t^{(\bar{\sigma} j)}\right)$ with the normalized metric. The index $\alpha$ runs over $\alpha=1,2, \ldots,\left(N^{2}-1\right)$ while the index $a$ runs over $a=1,2, \ldots,\left(M^{2}-1\right)$. The fundamental indices $\rho$ and $j$ run over $\rho=1,2, \ldots, N$ and $j=1,2, \ldots, M$ while the antifundamental indices $\bar{\sigma}$ and $\bar{i}$ run over $\bar{\sigma}=1,2, \ldots, N$ and $\bar{i}=1,2, \ldots, M$. The $f$ and $d$ symbols can be obtained from the above generators. The $S U(N+M)$ currents of spin-1 in the numerator satisfy the standard OPEs.

The $N M$ complex fermions of spin- $\frac{1}{2}$ in the numerator satisfy the following OPE

$\psi^{(\rho \bar{i})}(z) \psi^{(\bar{\sigma} j)}(w)=\frac{1}{(z-w)} \delta^{\rho \bar{\sigma}} \delta^{j \bar{i}}+\cdots$.

We introduce the $S U(N)$ currents, the $S U(M)$ currents and $U(1)$ current from these complex fermions living in the $S O(2 N M)_{1}$ factor as follows [30]:

$$
\begin{aligned}
& J_{f}^{\alpha} \equiv t_{\rho \bar{\sigma}}^{\alpha} \delta_{j \bar{i}} \psi^{(\rho \bar{i})} \psi^{(\bar{\sigma} j)}, \quad J_{f}^{a} \equiv-t_{j \bar{i}}^{a} \delta_{\rho \bar{\sigma}} \psi^{(\rho \bar{i})} \psi^{(\bar{\sigma} j)}, \\
& J_{f}^{u(1)} \equiv \delta_{\rho \bar{\sigma}} \delta_{j \bar{i}} \psi^{(\rho \bar{i})} \psi^{(\bar{\sigma} j)} .
\end{aligned}
$$

The appropriate contractions between the indices are taken. We can also choose the second currents with positive sign. Then the currents in the denominator of the coset (2.1) are given by

$$
J^{\alpha}+J_{f}^{\alpha}, \quad \sqrt{M N(M+N)} J^{u(1)}+(M+N) J_{f}^{u(1)},
$$

together with (2.3). Note that the level for the $J_{f}^{\alpha}$ is given by $M$ and the level for the $J_{f}^{u(1)}$ is given by $M N$ by using (2.2). See also Appendix A.2. Compared with the bosonic coset 
(1.1), the levels in the denominator are increased by $M$ and $N M(N+M)^{2}$ respectively. Note that the level for $J^{\alpha}$ and $J^{u(1)}$ is given by $k$.

The role of $J_{f}^{a}$ will appear in the next subsection. Note that the OPEs between any two different currents in (2.3) are regular. ${ }^{8}$ See also Appendix A.2. With the help of complex fermions, we can construct the currents of half-integer spin which are necessary to obtain the supersymmetric theory.

\subsubsection{The $\mathcal{N}=2$ superconformal algebra}

The stress energy tensor by Sugawara construction is the difference between the stress energy tensor in the numerator and the one in the denominator and is given by

$$
\begin{aligned}
T= & \frac{1}{2(k+M+N)}\left[J^{\alpha} J^{\alpha}+J^{a} J^{a}+\delta_{\rho \bar{\sigma}} \delta_{j \bar{i}} J^{(\rho \bar{i})} J^{(\bar{\sigma} j)}\right. \\
& \left.+\delta_{\rho \bar{\sigma}} \delta_{j \bar{i}} J^{(\bar{\sigma} j)} J^{(\rho \bar{i})}+J^{u(1)} J^{u(1)}\right] \\
& -\frac{1}{2}\left[\delta_{\rho \bar{\sigma}} \delta_{j \bar{i}} \psi^{(\rho \bar{i})} \partial \psi^{(\bar{\sigma} j)}-\delta_{\rho \bar{\sigma}} \delta_{j \bar{i}} \partial \psi^{(\rho \bar{i})} \psi^{(\bar{\sigma} j)}\right] \\
& -\frac{1}{2(k+M+N)}\left[\left(J^{\alpha}+J_{f}^{\alpha}\right)\left(J^{\alpha}+J_{f}^{\alpha}\right)\right. \\
& +\left(J^{u(1)}+\sqrt{\frac{M+N}{M N}} J_{f}^{u(1)}\right) \\
& \left.\times\left(J^{u(1)}+\sqrt{\frac{M+N}{M N}} J_{f}^{u(1)}\right)\right] .
\end{aligned}
$$

Compared with the bosonic case [3], ${ }^{9}$ due to the presence of the $S O(2 N M)_{1}$ factor in the numerator, its contribution appears in the second line of (2.5). The quantity $(k+M+N)$ in the first line can be interpreted as the sum of the level $k$ and $(N+M)$ of $S U(N+M)$. There exists a common factor $\frac{1}{2(k+M+N)}$ in the stress energy tensor of the denominator. For the $S U(N)$ current in (2.4), the quantity $(k+M+N)$ can be regarded as the sum of the level $(k+M)$ and the $N$ of $S U(N)$. When we multiply $M N(M+N)$ in the third line of (2.5) and divide it, then the corresponding level provides the overall factor $\frac{1}{2 N M(M+N)(k+M+N)}$ for the $U(1)$ current (2.4) in the denominator (2.4). In other words, the level can be identified with $N M(M+N)(k+M+N)$.

\footnotetext{
${ }^{8}$ It is obvious that the OPEs between the bosonic currents $\left(J^{\alpha}, J^{a}, J^{u(1)}, J^{(\rho \bar{i})}, J^{(\bar{\sigma} j)}\right)$ and the currents in (2.3) are regular.

9 The bosonic stress energy tensor is given by $T_{\text {boson }}=$ $\frac{1}{2(k+N+M)}\left[J^{\alpha} J^{\alpha}+J^{a} J^{a}+\delta_{\rho \bar{\sigma}} \delta_{j \bar{i}} J^{(\rho \bar{i})} J^{(\bar{\sigma} j)}+\delta_{\rho \bar{\sigma}} \delta_{j \bar{i}} J^{(\bar{\sigma} j)} J^{(\rho \bar{i})}+\right.$ $\left.J^{u(1)} J^{u(1)}\right]-\frac{1}{2(k+N)} J^{\alpha} J^{\alpha}-\frac{1}{2 k} J^{u(1)} J^{u(1)}$.
}

Then it is straightforward to compute the central charge for the supersymmetric coset model

$$
\begin{aligned}
c & =\frac{3 M N k}{(k+M+N)}+\frac{(k+N)\left(M^{2}-1\right)}{(k+M+N)} \\
& =\frac{\left(k M^{2}+3 k M N-k+M^{2} N-N\right)}{(k+M+N)} .
\end{aligned}
$$

When we have further $S U(M)_{k+N}$ factor in the denominator of (1.2), then the central charge is given by the first contribution of the first relation in (2.6). According to the observation of [31], this can be obtained from the level $k$ of $S U(N+M)$, the dual Coxeter number $(N+M)$ of $S U(N+M)$ and the dimension $2 N M$ of $\frac{S U(N+M)}{S U(N) \times S U(M) \times U(1)}$. See also [37,39]. The corresponding current of $S U(M)_{k+N}$ factor is given by $\left(J^{a}+J_{f}^{a}\right)$ and the level of $J_{f}^{a}$ is $N$. Therefore, the total level is $(k+N)$. By adding $S U(M)_{k+N}$ factor in the previous coset, we obtain the above coset (2.1). Then the central charge for the $S U(M)_{k+N}$ factor is given by the second contribution of the first relation in (2.6). ${ }^{10}$

By taking the product of the above complex fermions and the spin 1 currents transforming $(\mathbf{N}, \overline{\mathbf{M}})$ or $(\overline{\mathbf{N}}, \mathbf{M})$ with the appropriate contractions between the indices, we obtain the spin- $\frac{3}{2}$ currents [30] of $\mathcal{N}=2$ superconformal algebra as follows:

$\begin{aligned} G^{+} & \equiv \delta_{\rho \bar{\sigma}} \delta_{j \bar{i}} J^{(\rho \bar{i})} \psi^{(\bar{\sigma} j)}, \\ G^{-} & \equiv \delta_{\rho \bar{\sigma}} \delta_{j \bar{i}} \psi^{(\rho \bar{i})} J^{(\bar{\sigma} j)} .\end{aligned}$

It turns out that the remaining spin 1 current of $\mathcal{N}=2$ superconformal algebra is described by

$K \equiv \frac{1}{(k+M+N)}\left(\sqrt{\frac{M+N}{M N}} M N J^{u(1)}-k J_{f}^{u(1)}\right)$.

Note that the currents (2.5), (2.7) and (2.8) of the $\mathcal{N}=2$ superconformal algebra

$\left(K, G^{+}, G^{-}, T\right)$

are regular in the OPEs between these and the currents (2.4) of the denominator of the coset.

In Appendix A, we present some OPEs between the spin $\frac{1}{2}$ operators and the spin 1 operators, some OPEs between

\footnotetext{
${ }^{10}$ We can compute the central charges for the group $G=S U(N+$ $M)_{k} \times S O(2 N M)_{1}$ and for the group $H=S U(N)_{k+M} \times$ $U(1)_{N M(N+M)(k+M+N)}$ directly. For the former, we have the contributions from each factor $c_{G}=\frac{k\left((M+N)^{2}-1\right)}{k+(M+N)}+\frac{1}{1+(2 N M-2)} \frac{1}{2} 2 N M(2 N M-$ 1). For the latter $c_{H}=\frac{(k+M)\left(N^{2}-1\right)}{(k+M)+N}+\frac{N M(N+M)(k+M+N)}{N M(N+M)(k+M+N)+0}$ from each contribution. Then we obtain that the central charge (2.6) is given by their difference as follows: $c=c_{G}-c_{H}$. By using the property of $T(z) T_{H}(w)=0+\cdots$ where $T=T_{G}-T_{H}$, we confirm that the OPE $T(z) T(w)$ can be reduced to the OPE of $T_{G}(z) T_{G}(w)-T_{H}(z) T_{H}(w)$ $[77,78]$.
} 
the spin 1 operators, the OPEs between the spin $\frac{1}{2}, 1$ operators and the currents (2.9), and the complete OPEs of the $\mathcal{N}=2$ superconformal algebra. As noted by [30], there is a difference between the $\mathcal{N}=2$ superconformal algebra we are using in this paper and the standard $\mathcal{N}=2$ superconformal algebra for general $M(\neq 1)$ : (i) the normalizations in the spin- $1, \frac{3}{2}$ currents are different and (ii) in the OPE of $G^{+}(z) G^{-}(w)$, the stress energy tensor term has an additional term, $\left(J^{a}+J_{f}^{a}\right)^{2}$. For $M=1$ case, this additional term vanishes and we obtain the standard $\mathcal{N}=2$ superconformal algebra.

\subsubsection{The so far known currents}

In the bosonic coset model, the currents contain the $J^{a}$ of spin-1, the $K^{a}$ of spin-2, the $P^{a}$ of spin-3 and the $W^{(3)}$ of spin-3. They are all primary under the corresponding stress energy tensor appearing in the footnote 9 . The question is whether they are primary under the above stress energy tensor (2.5) or not. Recall that the bosonic stress energy tensor consists of the first line of (2.5) in addition to $J^{\alpha} J^{\alpha}$ and $J^{u(1)} J^{u(1)}$ terms. By construction, the above primary currents do not have any singular terms in the OPEs between them and these quadratic terms. Then we can regard the first line of (2.5) as the bosonic stress energy tensor. Let us look at the second and third lines of (2.5). They are either purely complex fermions dependent terms, $J^{\alpha}$ dependent term, $J^{u(1)}$ dependent term, $J^{\alpha}$ with complex fermions, or $J^{u(1)}$ with complex fermions. It is clear to observe that the above primary currents consisting of purely bosonic operators do not produce any nontrivial singular terms when we calculate the OPEs between them and the second and third lines of (2.5). This implies that the above primary currents with bosonic stress energy tensor are primary also under the above stress energy tensor (2.5).

Furthermore, because the above primary currents do not contain the complex fermions, it is obvious that the regular conditions for these currents with the operators (2.4) in the denominator hold. Then all the (quasi)primary currents in the bosonic coset model can play the role of primary operators under the stress energy tensor (2.5). However, we should check whether they transform under the spin- $\frac{3}{2}$ currents in (2.7) and the spin-1 current (2.8) or not.

So far, there exist the following currents $[3,12,30]$

$\operatorname{spin}-1: J^{a}, \quad \operatorname{spin}-2: K^{a}, \quad \operatorname{spin}-3: P^{a}, \quad \cdots$, spin-1 $: K, \quad \operatorname{spin}-\frac{3}{2}: G^{+}, G^{-}, \quad \operatorname{spin}-2: T$,

$$
\text { spin-3: } W^{(3)}, \cdots \text {. }
$$

We would like to construct the additional higher spin currents of integer or half-integer spins in terms of the coset operators $\left(J^{\alpha}, J^{a}, J^{u(1)}, J^{(\rho \bar{i})}, J^{(\bar{\sigma} j)}\right)$ and $\left(\psi^{(\rho \bar{i})}, \psi^{(\bar{\sigma} j)}\right)$. Moreover, by using their OPEs, we determine the possible new higher spin currents. We will obtain $\mathcal{N}=2$ supersymmetric currents corresponding to the above $K^{a}, W^{(3)}$ and $P^{a}$. Although these currents do not belong to the extension of $\mathcal{N}=2$ superconformal algebra we are describing in this paper, they can provide how we can construct their $\mathcal{N}=2$ supersymmetric versions explicitly by using the currents of $\mathcal{N}=2$ superconformal algebra.

\subsection{The nonsinglet currents of spins $\left(1, \frac{3}{2}, \frac{3}{2}, 2\right)$}

In [30], the three currents of this multiplet were obtained. We can consider the $S U(M)$ current in (2.3) as the lowest component of this multiplet. This current has the adjoint index $a$. In order to have the spin- $\frac{3}{2}$ current which has an index $a$, we use the generator of $S U(M)$ by making the product of spin- 1 current and spin- $\frac{1}{2}$ operator with the appropriate contractions of the indices. It turns out that in [30] there exist the nonsinglet spin- $\frac{3}{2}$ currents

$\begin{aligned} G^{+, a} & \equiv-t_{j \bar{i}}^{a} \delta_{\rho \bar{\sigma}} J^{(\rho \bar{i})} \psi^{(\bar{\sigma} j)}, \\ G^{-, a} & \equiv-t_{j \bar{i}}^{a} \delta_{\rho \bar{\sigma}} \psi^{(\rho \bar{i})} J^{(\bar{\sigma} j)} .\end{aligned}$

Then it is natural to describe the following $\mathcal{N}=2$ multiplet

$\left(J_{f}^{a}, G^{+a}, G^{-a}, ?\right)$.

In next section, we will determine the last component of this multiplet.

\subsection{The regular condition}

By construction, all the currents should satisfy the regular conditions in the OPEs between them and the currents in the denominator of the coset (2.1). All the bosonic currents in the coset model of [3] satisfy automatically because the additional currents in (2.3) come from the complex fermions. For example, the spin- 1 current in (2.10) satisfies the trivial OPEs

$$
\begin{aligned}
& J^{a}(z)\left(J^{u(1)}+\sqrt{\frac{M+N}{M N}} J_{f}^{u(1)}\right)(w)=0+\cdots, \\
& J^{a}(z)\left(J^{\alpha}+J_{f}^{\alpha}\right)(w)=0+\cdots .
\end{aligned}
$$

If we determine the currents from the coset fields from the beginning by taking the multiple product between them and introducing the arbitrary coefficients to be fixed, then it is necessary to check the regular conditions like as (2.13) explicitly. However, when we are calculating some OPEs between the known currents which satisfy the regular conditions already, we do not need to check them at each step because some composite operators appearing in the right hand sides of these OPEs satisfy these regular conditions automatically. At the final stage, it is better to check these conditions for consistency checks. 
Therefore, the lowest singlet and nonsinglet $\mathcal{N}=2$ multiplets are classified by (2.9) and (2.12). The former is the $\mathcal{N}=2$ supersymmetric extension of the stress energy tensor of the bosonic coset model.

\section{The nonsinglet multiplet of spins $\left(1, \frac{3}{2}, \frac{3}{2}, 2\right)$}

After identifying the last component of the $\mathcal{N}=2$ multiplet correctly, we present the OPEs between the generators of $\mathcal{N}=2$ superconformal algebra and this $\mathcal{N}=2$ multiplet. Moreover, the OPEs between the supersymmetry generators and the spin- 2 current $K^{a}$ obtained in the bosonic coset model are given.

\subsection{The spin-2 current}

The simplest $\mathcal{N}=2$ multiplet is described in (2.12). In this section, we would like to obtain the last component of this multiplet in terms of coset fields explicitly. Later we will also consider their OPEs and determine their algebraic structures in Sect. 6.

How do we obtain the last component of the multiplet (2.12)? According to the primary condition of the $\mathcal{N}=2$ multiplet under the multiplet of (2.9) in the $\mathcal{N}=2$ superconformal algebra, we can use the OPEs between $G^{ \pm}(z)$ and $G^{\mp, a}(w)$ and look at the first order pole. See Appendix B. Based on the explicit expressions of (2.7) and (2.11), we can compute the OPE between $G^{+}(z)$ and $G^{-, a}(w)$ explicitly.

After subtracting the descendant terms from the first order pole of the OPE $G^{+}(z) G^{-, a}(w)$, the primary spin-2 current is ${ }^{11}$

$$
\begin{aligned}
W^{+(2), a}= & -t_{j \bar{i}}^{a} \delta_{\rho \bar{\sigma}} J^{(\rho \bar{i})} J^{(\bar{\sigma} j)}+k t_{j \bar{i}}^{a} \delta_{\rho \bar{\sigma}} \psi^{(\rho \bar{i})} \partial \psi^{(\bar{\sigma} j)} \\
& -\sqrt{\frac{M+N}{M N}} J^{u(1)} J_{f}^{a} \\
& +t_{\rho \bar{\sigma}}^{\alpha} t_{j \bar{i}}^{a} J^{\alpha} \psi^{(\rho \bar{i})} \psi^{(\bar{\sigma} j)}-\frac{1}{M} J^{a} J_{f}^{u(1)} \\
& +\frac{1}{2}(i f+d)^{b a c} J^{b} J_{f}^{c} \\
& -\frac{N}{2} \partial J^{a}+\frac{k}{2} \partial J_{f}^{a} \equiv-\frac{1}{2} K^{a}+\hat{W}^{+(2), a},
\end{aligned}
$$

\footnotetext{
11 After extracting the bosonic spin-2 current from this spin2 current, we obtain the following spin-2 current $\hat{W}^{+(2), a}=$ $k t_{j \bar{i}}^{a} \delta_{\rho \bar{\sigma}} \psi^{(\rho \bar{i})} \partial \psi^{(\bar{\sigma} j)}-\sqrt{\frac{M+N}{M N}} J^{u(1)} J_{f}^{a}+t_{\rho \bar{\sigma}}^{\alpha} t_{j \bar{i}}^{a} J^{\alpha} \psi^{(\rho \bar{i})} \psi^{(\bar{\sigma} j)}-$ $\frac{1}{M} J^{a} J_{f}^{u(1)}+\frac{1}{2}(i f+d)^{b a c} J^{b} J_{f}^{c}+\frac{k}{2} \partial J_{f}^{a}+\left(-\frac{N}{2(2 k+M)} d^{a b c} J^{b} J^{c}+\right.$ $\left.\frac{N}{k} \sqrt{\frac{M+N}{M N}} J^{a} J^{u(1)}\right)$ which is a primary. In Sect. 6, we will use the last relation of (3.1) in order to apply the results in [12].
}

where the spin- 2 current obtained in the bosonic coset model is given by [3]

$$
\begin{aligned}
K^{a}= & \delta_{\rho \bar{\sigma}} t_{j \bar{i}}^{a}\left(J^{(\rho \bar{i})} J^{(\bar{\sigma} j)}+J^{(\bar{\sigma} j)} J^{(\rho \bar{i})}\right) \\
& -\frac{N}{(M+2 k)} d^{a b c} J^{b} J^{c} \\
& +\frac{2 N}{k} \sqrt{\frac{M+N}{M N}} J^{a} J^{u(1)} .
\end{aligned}
$$

We realize that the field contents in (3.1) cannot be written in terms of the known currents in (2.10) and (2.12) due to the first five terms. In particular, the fourth term of (3.1) looks like the first term of spin-3 current $P^{a}$ appearing in (2.10) in the sense that $J^{(\bar{\rho} \bar{i})} J^{(\bar{\sigma} j)}$ is replaced by $\psi^{(\rho \bar{i})} \psi^{(\bar{\sigma} j)}$ and other factors remain unchanged. The reason why we write down the above spin- 2 current in terms of the known previous spin-2 current and other piece is that when we calculate some OPEs including this current, we can use the previous result found in [12]. For example, in the OPE between $W^{+(2), a}(z)$ and $W^{+(2), b}(w)$, it is rather complicated to determine the OPE between the first term of (3.1) and itself. Instead, we can change those first term by using (3.2) in terms of $J^{a}, J^{u(1)}$ and $K^{a}$. We will see the details in Sect. 6 .

3.2 The OPEs with the currents of $\mathcal{N}=2$ superconformal algebra

Once we have determined the four currents in the multiplet,

$\left(J_{f}^{a}, G^{+, a}, G^{-, a}, W^{+(2), a}\right)$,

we need to check whether they are really the components of $\mathcal{N}=2$ multiplet or not. It is straightforward to calculate the following OPEs with the help of (2.3), (2.8), (2.11) and (3.1)

$$
\begin{aligned}
K(z) J_{f}^{a}(w) & =0+\cdots, \\
K(z) G^{ \pm, a}(w) & = \pm \frac{1}{(z-w)} G^{ \pm, a}(w)+\cdots, \\
K(z) W^{+(2), a}(w) & =\frac{1}{(z-w)^{2}}\left[N J^{a}-k J_{f}^{a}\right](w)+\cdots .
\end{aligned}
$$

When we compare the ones in Appendix B with these OPEs (3.4), the last OPE contains the additional term $J^{a}$ in the second order pole of the right hand side. There are two ways to fix this inconsistency by considering the above spin-2 current with some modifications in order to have $J_{f}^{a}(w)$ in the above second order pole or by taking the spin-1 current as $\left(N J^{a}-k J_{f}^{a}\right)$ rather than the previous $J_{f}^{a}$. Recall that the OPE between $K(z)$ and $J^{a}(w)$ is regular because it is obvious to see that the OPE between the spin-1 current $J^{a}$ and the spin- $1 J_{f}^{u(1)}$ having complex fermions does not have any singular terms and moreover it is known that $J^{a}(z) J^{u(1)}(w)=0+\cdots$ from the observation of [3]. This 
implies that the above linear combination of spin- 1 current satisfies the corresponding OPE which is the first one in (3.4). Then we will have the right property in Appendix B.

However, we will take the lowest component of the multiplet as $J_{f}^{a}$ rather than $\left(N J^{a}-k J_{f}^{a}\right)$ as in (3.3). One of the reasons why we do not consider this linear combination as the lowest component of the above multiplet is that it is better to treat $J^{a}$ and $J_{f}^{a}$ separately when we decide which composite operators are written in terms of the known currents or not. Otherwise we should replace all the $J_{f}^{a}$ term by adding $J^{a}$ term in order to preserve the above linear combination in all the composite operators. ${ }^{12} \mathrm{We}$ observe that the combination of $\left(N J^{a}-k J_{f}^{a}\right)$ will appear at many places of the OPEs we will calculate later.

Let us describe the OPEs between the spin- $\frac{3}{2}$ current of $\mathcal{N}=2$ superconformal algebra and the currents of the multiplet in $(3.3)^{13}$

$$
\begin{aligned}
G^{+}(z) J_{f}^{a}(w)= & \frac{1}{(z-w)} G^{+, a}(w)+\cdots, \\
G^{+}(z) G^{+, a}(w)= & 0+\cdots, \\
G^{+}(z) G^{-, a}(w)= & \frac{1}{(z-w)^{2}}\left[N J^{a}-k J_{f}^{a}\right](w) \\
& +\frac{1}{(z-w)}\left[\frac{1}{2}\left(N \partial J^{a}-k \partial J_{f}^{a}\right)\right. \\
& \left.+W^{+(2), a}\right](w)+\cdots, \\
G^{+}(z) W^{+(2), a}(w)= & \frac{1}{(z-w)^{2}} \frac{3}{2}(k+N) G^{+, a}(w) \\
& +\frac{1}{(z-w)} \frac{1}{2}(k+N) \partial G^{+, a}(w)+\cdots .
\end{aligned}
$$

By changing the sign of $J_{f}^{a}(w)$ (and the remaining currents are the same) we obtain the standard OPE of $G^{+}(z) J_{f}^{a}(w)$ which leads to the minus sign of the right hand side of this OPE. See also Appendix B. Note that there is a term of $J^{a}(w)$ in the second order pole of $G^{+}(z) G^{-, a}(w)$ as before. From this OPE, we have obtained the nonsinglet spin-2 current we mentioned before. In the last OPE, the numerical value in the right hand side has an extra factor $(k+N)$ when we compare with the one in Appendix B. ${ }^{14}$

\footnotetext{
12 Note that all the $\mathcal{N}=2$ multiplets in this paper do not satisfy the properties in Appendix B. In other words, some of the coefficients appearing in Appendix B appear differently and the vanishing coefficients in the higher order poles in Appendix B appear nontrivially.

13 For convenience, we present the following OPEs $J^{a}(z) G^{ \pm}(w)= \pm \frac{1}{(z-w)} G^{ \pm, a}(w)+\cdots, \quad J^{\alpha}(z) G^{+}(w)=$ $\frac{1}{(z-w)} t_{\rho \bar{\sigma}}^{\alpha} \delta_{j \bar{k}} \psi^{(\bar{\sigma} j)} J^{(\rho \bar{k})}(w)+\cdots \quad$ and $J^{\alpha}(z) G^{-}(w)=$ $-\frac{1}{(z-w)} t_{\rho \bar{v}}^{\alpha} \delta_{m \bar{m}} \psi^{(\rho \bar{m})} J^{(\bar{v} m)}(w)+\cdots$ which will be used in the last OPE of (3.5)

${ }^{14}$ In the last OPE, we use the following identity $t_{j \bar{i}}^{a} \delta_{k \bar{l}} \delta_{\rho \bar{\mu}} \delta_{\tau \bar{\sigma}}$ $\psi^{(\bar{\sigma} j)} \psi^{(\rho \bar{i})} \psi^{(\bar{\mu} k)} J^{(\tau \bar{l})}=\frac{1}{2}(i f+d)^{b a c} G^{+, b} J_{f}^{c}-\frac{1}{M} G^{+} J_{f}^{a}$
}

Similarly, we obtain the following OPEs

$$
\begin{aligned}
G^{-}(z) J_{f}^{a}(w)= & -\frac{1}{(z-w)} G^{-, a}(w)+\cdots, \\
G^{-}(z) G^{+, a}(w)= & \frac{1}{(z-w)^{2}}\left[-N J^{a}+k J_{f}^{a}\right](w) \\
& +\frac{1}{(z-w)}\left[\frac{1}{2}\left(-N \partial J^{a}+k \partial J_{f}^{a}\right)\right. \\
& \left.+W^{+(2), a}+i f^{a b c} J^{b} J_{f}^{c}\right](w)+\cdots, \\
G^{-}(z) G^{-, a}(w)= & +\cdots, \\
G^{-}(z) W^{+(2), a}(w)= & \frac{1}{(z-w)^{2}} \frac{1}{2}(3 k+2 M+3 N) G^{-, a}(w) \\
& +\frac{1}{(z-w)}\left[\frac{1}{3} \frac{1}{2}(3 k+2 M+3 N) \partial G^{-, a}\right. \\
& +i f^{a b c} J^{b} G^{-, c}-i f^{a b c} G^{-, b} J_{f}^{c} \\
& \left.-\frac{M}{3} \partial G^{-, a}\right](w)+\cdots
\end{aligned}
$$

By changing the sign of $J_{f}^{a}(w)$ as before, we obtain the standard OPE of $G^{-}(z) J_{f}^{a}(w)$ in Appendix B. There exists a term of $J^{a}(w)$ in the second order pole of $G^{-}(z) G^{+, a}(w)$. In the last OPE of (3.6), we do not combine with the first and the fourth terms in the first order pole. ${ }^{15}$ The last three terms of the last OPE (which is primary under the stress energy tensor) are written in terms of the known currents in (2.10). ${ }^{16}$

We can check that each current in the multiplet we are considering in this subsection satisfies the primary condition under the stress energy tensor (2.5) as follows:

$$
\begin{aligned}
T(z) J_{f}^{a}(w)= & \frac{1}{(z-w)^{2}} J_{f}^{a}(w) \\
& +\frac{1}{(z-w)} \partial J_{f}^{a}(w)+\cdots, \\
T(z) G^{ \pm, a}(w)= & \frac{1}{(z-w)^{2}} \frac{3}{2} G^{ \pm, a}(w) \\
& +\frac{1}{(z-w)} \partial G^{ \pm, a}(w)+\cdots,
\end{aligned}
$$

Footnote 14 continued

$-\frac{1}{M} G^{+, a} J_{f}^{u(1)}$ by using the rearrangement lemma in $[78,79]$. Here the $J_{f}^{u(1)}$ term is canceled by other term in the first order pole of the last OPE. Then we are left with the known currents finally.

15 In all the OPEs in this paper, we intentionally put the exact coefficients coming from the descendant terms in the right hand sides of the OPEs without simplifying them.

16 In the last OPE, the following identity is used $t_{j \bar{i}}^{a} \delta_{\rho \bar{\mu}} \delta_{\tau \bar{\sigma}} \delta_{k \bar{l}} \psi^{(\rho \bar{i})} \psi^{(\bar{\sigma} j)} \psi^{(\tau \bar{l})} J^{(\bar{\mu} k)}=\frac{1}{M} J_{f}^{a} G^{-}+\frac{1}{M} J_{f}^{u(1)} G^{-, a}-$ $\frac{1}{2}(i f+d)^{a b c} J_{f}^{c} G^{-, b}$ with the help of [78,79] again. The $J_{f}^{u(1)}$ term we do not want to have can be canceled by other term appearing in the first order pole of the last OPE of (3.6). 


$$
\begin{aligned}
T(z) W^{+(2), a}(w)= & \frac{1}{(z-w)^{2}} 2 W^{+(2), a}(w) \\
& +\frac{1}{(z-w)} \partial W^{+(2), a}(w)+\cdots .
\end{aligned}
$$

Of course, it is rather nontrivial to check these OPEs (3.7) for generic $k, N$ and $M$ by hands but we can check them for fixed $N$ and $M$ by using the Thielemans package [80]. When we notice the presence of higher order terms where the pole is greater than or equal to three, then we can try to do this checking by hands on the higher order terms. Usually, it is not necessary to check the first and second order poles of the OPEs. For the quasi primary operators, it is nontrivial to check the fourth order poles by hands.

As a consistency check, we can check that the currents satisfy the following regular conditions

$$
\begin{aligned}
& \left(J_{f}^{a}, G^{+, a}, G^{-, a}, W^{+(2), a}\right)(z)\left(J^{u(1)}\right. \\
& \left.\quad+\sqrt{\frac{M+N}{M N}} J_{f}^{u(1)}\right)(w)=0+\cdots, \\
& \left(J_{f}^{a}, G^{+, a}, G^{-, a}, W^{+(2), a}\right)(z)\left(J^{\alpha}+J_{f}^{\alpha}\right)(w)=0+\cdots .
\end{aligned}
$$

For example, the relative coefficients in (3.1) are fixed from these constraints and the primary condition under the stress energy tensor (2.5) (and its $\mathcal{N}=2$ extension).

\subsection{Some OPEs with the spin-2 current $K^{a}$}

It is clear that the OPE between $K(z)$ and the spin-2 current $K^{a}(w)$ does not contain the singular terms because the OPE between the $J^{u(1)}(z)$ and $K^{a}(w)$ is regular and the $K^{a}(w)$ does not have the complex fermions. Then the question is what are the OPEs between $G^{ \pm}(z)$ and $K^{a}(w) .{ }^{17}$

By using the explicit expressions of (2.7) and (3.2) we obtain

$$
\begin{aligned}
& G^{+}(z) K^{a}(w) \\
& =-\frac{1}{(z-w)^{2}} \frac{2\left(k^{2}-1\right)(2 k+M+N)}{k(2 k+M)} G^{+, a}(w) \\
& \quad+\frac{1}{(z-w)}\left[-\frac{1}{3} \frac{2\left(k^{2}-1\right)(2 k+M+N)}{k(2 k+M)} \partial G^{+, a}\right. \\
& \left.\quad+V^{+\left(\frac{5}{2}\right), a}\right](w)+\cdots .
\end{aligned}
$$

Note that the first order pole provides the spin- $\frac{5}{2}$ current which cannot be written in terms of the known currents (2.10)

\footnotetext{
17 The OPE between $K(z)$ and $K^{a}(w)$ is regular and the OPE between $T(z)$ and $K^{a}(w)$ satisfies the standard OPE for a primary field mentioned before.
}

and (3.1). The primary spin- $\frac{5}{2}$ current under the stress energy tensor (2.5), after subtracting the descendant term in the first order pole is

$$
\begin{aligned}
V^{+\left(\frac{5}{2}\right), a}= & -2(k+N) t_{j \bar{i}}^{a} \delta_{\rho \bar{\sigma}} \partial J^{(\rho \bar{i})} \psi^{(\bar{\sigma} j)} \\
& -\frac{2(k+N)}{k} \sqrt{\frac{M+N}{M N}} J^{u(1)} G^{+, a} \\
& -\frac{2}{k M}(k+M+N) J^{a} G^{+} \\
& -\left(i f-\frac{2 k+M+2 N}{2 k+M} d\right)^{a b c} J^{b} G^{+, c} \\
& +2 t_{\rho \bar{\sigma}}^{\alpha} t_{j \bar{i}}^{a} J^{\alpha} J^{(\rho \bar{i})} \psi^{(\bar{\sigma} j)} \\
& -\frac{2}{3} \frac{2\left(k^{2}-1\right)(2 k+M+N)}{k(2 k+M)} \partial G^{+, a} .
\end{aligned}
$$

Note that the first, second and fifth terms are the reasons why we cannot write down this current in terms of the known currents. Again, the field content of the fifth term looks like the fourth term of (3.1) in the sense that the spin- $\frac{1}{2}$ operator is replaced by the spin-1 current.

Similarly, we can determine the following OPE

$$
\begin{aligned}
G^{-} & (z) K^{a}(w) \\
= & -\frac{1}{(z-w)^{2}} \frac{2\left(k^{2}-1\right)(2 k+M+N)}{k(2 k+M)} G^{-, a}(w) \\
& +\frac{1}{(z-w)}\left[-\frac{1}{3} \frac{2\left(k^{2}-1\right)(2 k+M+N)}{k(2 k+M)} \partial G^{-, a}\right. \\
& \left.+V^{-\left(\frac{5}{2}\right), a}\right](w)+\cdots,
\end{aligned}
$$

where the primary spin- $\frac{5}{2}$ current is

$$
\begin{aligned}
V^{-\left(\frac{5}{2}\right), a}= & -2(k+N) t_{j \bar{i}}^{a} \delta_{\rho \bar{\sigma}} \partial J^{(\bar{\sigma} j)} \psi^{(\rho \bar{i})} \\
& +\frac{2(k+N)}{k} \sqrt{\frac{M+N}{M N}} J^{u(1)} G^{-, a} \\
& +\frac{2}{k M}(k+M+N) J^{a} G^{-} \\
& -\left(i f+\frac{2 k+M+2 N}{2 k+M} d\right)^{a b c} J^{b} G^{-, c} \\
& -2 t_{\rho \bar{\sigma}}^{\alpha} t_{j \bar{i}}^{a} J^{\alpha} J^{(\bar{\sigma} j)} \psi(\rho \bar{i}) \\
& -\frac{2}{3} \frac{2\left(k^{2}-1\right)(2 k+M+N)}{k(2 k+M)} \partial G^{-, a} .
\end{aligned}
$$

The field contents of (3.12) look similar to the one in (3.10). They will appear at many places in the OPEs we will consider later. Due to the presence of the new primary fields (3.10) and (3.12) for given OPEs, the bosonic spin-2 current $K^{a}$ by itself cannot play the role of the last component of the multiplet in (2.12). In other words, the right spin-2 current in 
(3.1) contains both fermionic dependent terms and bosonic current dependent terms in addition to $K^{a}$. If we remember the OPEs in Appendix B, the OPEs in (3.9) and (3.11) imply that the spin-2 current $K^{a}$ can be a candidate for the lowest component of other nonsinglet $\mathcal{N}=2$ multiplet which will be explained later if we succeed to eliminate the second order poles of (3.9) and (3.11). ${ }^{18}$

In summary, the equations (3.4), (3.5), (3.6) and (3.7) imply that the right hand sides of these OPEs contain the currents (3.3), the nonsinglet spin-1 current $J^{a}$ and their composite operators. As emphasized before, these OPEs do not satisfy Appendix B.

\section{The singlet multiplet of spins $\left(2, \frac{5}{2}, \frac{5}{2}, 3\right)$}

We would like to construct this multiplet starting from the singlet spin-3 current found in the bosonic coset model [3].

\subsection{Construction of lowest component}

Once we know any component of this multiplet, then the other three components can be determined, in principle, by using the $\mathcal{N}=2$ primary conditions described in Appendix B. Let us first consider how we obtain the lowest component of this $\mathcal{N}=2$ multiplet. ${ }^{19}$

There exists a singlet spin-3 current $W^{(3)}$ in the bosonic coset model and its expression in terms of coset fields is described by [3]

$$
\begin{aligned}
W^{(3)}= & b_{1} d^{\alpha \beta \gamma} J^{\alpha} J^{\beta} J^{\gamma}+b_{2} d^{a b c} J^{a} J^{b} J^{c} \\
& +b_{3} J^{u(1)} J^{u(1)} J^{u(1)}+b_{4} J^{\alpha} J^{\alpha} J^{u(1)} \\
& +b_{5} J^{a} J^{a} J^{u(1)}+b_{6} t_{\rho \bar{\sigma}}^{\alpha} \delta_{j \bar{i}} J^{\alpha}\left(J^{(\rho \bar{i})} J^{(\bar{\sigma} j)}\right. \\
& \left.+J^{(\bar{\sigma} j)} J^{(\rho \bar{i})}\right) \\
& +b_{7} \delta_{\rho \bar{\sigma}} t_{j \bar{i}}^{a} J^{a}\left(J^{(\rho \bar{i})} J^{(\bar{\sigma} j)}+J^{(\bar{\sigma} j)} J^{(\rho \bar{i})}\right) \\
& +b_{8} \delta_{\rho \bar{\sigma}} \delta_{j \bar{i}} J^{u(1)}\left(J^{(\rho \bar{i})} J^{(\bar{\sigma} j)}+J^{(\bar{\sigma} j)} J^{(\rho \bar{i})}\right)
\end{aligned}
$$

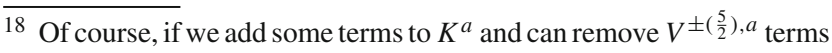
properly, then this modified spin-2 current can be the last component of the $\mathcal{N}=2$ multiplet of this section. See the last OPEs in (3.5) and (3.6).

19 Although we have realized that the spin-2 current $K^{a}$ will participate in the lowest component from the previous section, it is not clear how we can continue to calculate the additional terms by adding the possible composite operators of spin- 2 to the $K^{a}$ with an appropriate contraction in the indices.
}

$$
\begin{aligned}
& +b_{12} \delta_{\rho \bar{\sigma}} \delta_{j \bar{i}} \partial J^{(\rho \bar{i})} J^{(\bar{\sigma} j)} \\
& +b_{13} \delta_{\rho \bar{\sigma}} \delta_{j \bar{i}} \partial J^{(\bar{\sigma} j)} J^{(\rho \bar{i})}+b_{14} \partial^{2} J^{u(1)} .
\end{aligned}
$$

Here the relative coefficients are functions of $k, N$ and $M$ as follows:

$$
\begin{aligned}
b_{2} & =-\frac{N(k+N)(k+2 N)}{M(k+M)(k+2 M)} b_{1}, \\
b_{3} & =\sqrt{\frac{M+N}{M N}} \frac{2(k+N)(k+2 N)(M+N)}{k^{2} M} b_{1}, \\
b_{4} & =\sqrt{\frac{M+N}{M N}} \frac{6(k+N)}{k} b_{1}, \\
b_{5} & =\sqrt{\frac{M+N}{M N}} \frac{6 N(k+N)(k+2 N)}{k M(k+2 M)} b_{1}, \\
b_{6} & =-\frac{3(k+N)}{M} b_{1}, \\
b_{7} & =\frac{3(k+N)(k+2 N)}{M(k+2 M)} b_{1}, \\
b_{8} & =-\sqrt{\frac{M+N}{M N}} \frac{3(k+N)(k+2 N)}{k M} b_{1}, \\
b_{12} & =\frac{3(k+N)(k+2 N)}{M} b_{1}, \\
b_{13} & =-\frac{3(k+N)(k+2 N)}{M} b_{1}, \\
b_{14} & =-\sqrt{\frac{M+N}{M N}} \frac{N(k+N)(k+2 N) b_{1} .}{M},
\end{aligned}
$$

Although we add the spin-1 currents coming from the complex fermions to the ones in the bosonic coset model studied in [3], the regular conditions with the coset fields in the coset (2.1) hold as follows:

$$
\begin{aligned}
& W^{(3)}(z)\left(J^{u(1)}+\sqrt{\frac{M+N}{M N}} J_{f}^{u(1)}\right)(w)=0+\cdots, \\
& W^{(3)}(z)\left(J^{\alpha}+J_{f}^{\alpha}\right)(w)=0+\cdots,
\end{aligned}
$$

because the OPEs between the spin-3 current $W^{(3)}$ (consisting of purely bosonic fields) and these spin-1 currents (consisting of purely fermionic fields) do not have any singular terms as in (3.8).

We need to calculate the OPEs between the supersymmetry generators of the $\mathcal{N}=2$ superconformal algebra and the above singlet spin-3 current in order to determine its superpartners. Because the spin- $\frac{3}{2}$ currents consist of the spin$1, \frac{1}{2}$ operators transforming as $(\mathbf{N}, \overline{\mathbf{M}})$ or $(\overline{\mathbf{N}}, \mathbf{M})$, we should first calculate the OPEs between the spin-1 currents and the spin-3 current $W^{(3)}$. Note that it is obvious to see that the OPEs between the spin- $\frac{1}{2}$ operator and $W^{(3)}$ are regular. In Appendix C, we present some relevant OPEs between the spin-1 current and the singlet spin-3 current.

It turns out that after extracting the OPE $W^{(3)}(z) G^{ \pm}(w)$ and then changing the order of the two currents we have 


$$
\begin{aligned}
& G^{ \pm}(z) W^{(3)}(w) \\
& =\mp \frac{1}{(z-w)^{3}} \frac{\left(k^{2}-1\right)\left(k^{2}-4\right)(k+M+N)(2 k+M+N)(3 k+2 M+2 N)}{k^{2} M(k+M)(k+2 M)} b_{1} G^{ \pm}(w) \\
& \pm \frac{1}{(z-w)^{2}} G^{ \pm\left(\frac{5}{2}\right), 0}(w)+\mathcal{O}\left(\frac{1}{(z-w)}\right) .
\end{aligned}
$$

We do not specify the first order poles of (4.4) here. They can be written explicitly from the results of Appendix C. Because there are no descendant terms associated with the spin- $\frac{3}{2}$ currents at the second order pole, the primary singlet spin- $\frac{5}{2}$ currents appear in the second order pole.

One of them is given by

$$
\begin{aligned}
G^{+\left(\frac{5}{2}\right), 0}= & \frac{\left(k^{2}-4\right)(k+M+N)(3 k+2 M+2 N)}{k M(k+2 M)} b_{1} \\
& \times\left[-3 t_{\sigma \bar{\sigma}}^{\alpha} \delta_{j \bar{i}} J^{\alpha} \psi^{(\bar{\sigma} j)} J^{(\sigma \bar{i})}\right. \\
& -\frac{3}{(k+M)}(k+N) J^{a} G^{+, a} \\
& -\frac{3}{k} \sqrt{\frac{M+N}{M N}}(k+N) J^{u(1)} G^{+} \\
& +3(k+N) \delta_{\rho \bar{\sigma}} \delta_{j \bar{i}} \psi^{(\bar{\sigma} j)} \partial J^{(\rho \bar{i})} \\
& \left.-\frac{\left(k^{2}-1\right)(2 k+M+N)}{k(k+M)} \partial G^{+}\right] .
\end{aligned}
$$

Due to the first, third and fourth terms, we cannot rewrite (4.5) in terms of previous known currents we have discussed so far. ${ }^{20}$ The field contents look similar to the one $V^{+\left(\frac{5}{2}\right), a}$ (3.10). There is no $f$ or $d$ symbols in (4.5) because this current should be a singlet field. We can easily check that the first term comes from the sixth term of (4.1).

The other is given by

$$
\begin{aligned}
G^{-\left(\frac{5}{2}\right), 0}= & \frac{\left(k^{2}-4\right)(k+M+N)(3 k+2 M+2 N)}{k M(k+2 M)} b_{1} \\
& \times\left[3 t_{\sigma \bar{\sigma}}^{\alpha} \delta_{j \bar{i}} J^{\alpha} \psi^{(\sigma \bar{i})} J^{(\bar{\sigma} \bar{j})}\right. \\
& +\frac{3}{(k+M)}(k+N) J^{a} G^{-, a} \\
& +\frac{3}{k} \sqrt{\frac{M+N}{M N}}(k+N) J^{u(1)} G^{-} \\
& +3(k+N) \delta_{\rho \bar{\sigma}} \delta_{j \bar{i}} \psi^{(\rho \bar{i})} \partial J^{(\bar{\sigma} j)} \\
& \left.-\frac{\left(k^{2}-1\right)(2 k+M+N)}{k(k+M)} \partial G^{-}\right] .
\end{aligned}
$$

$\overline{20}$ The overall factor $b_{1}$ appears in this expression by using (4.2) for the $W^{(3)}$.
The field contents of (4.6) look similar to the one $V^{-\left(\frac{5}{2}\right), a}$ (3.12). Again the first term comes from the sixth term of (4.1). Of course, after we determine the lowest component of this multiplet later, we should check whether the above two primary spin- $\frac{5}{2}$ currents are really the elements of this multiplet under the action of the generators of $\mathcal{N}=2$ superconformal algebra based on Appendix B. In general, we expect that the spin- $\frac{5}{2}$ currents obtained by $W^{(3)}$ from the bosonic coset model will be different from the ones determined by the corresponding currents in $\mathcal{N}=2$ multiplet. However, it turns out that they are equivalent to each other. In next section, we will observe some examples where this is not the case.

From Appendix B, the second order pole in the OPE of either $G^{-}(z) G^{+\left(\frac{5}{2}\right), 0}(w)$ or $G^{+}(z) G^{-\left(\frac{5}{2}\right), 0}(w)$ leads to the following primary singlet spin-2 current, which is the lowest component of $\mathcal{N}=2$ multiplet in this section ${ }^{21}$

$$
\begin{aligned}
W^{-(2), 0}= & \frac{\left(k^{2}-4\right)(k+M+N)(3 k+2 M+2 N)}{k M(k+2 M)} b_{1} \\
& \times\left[-\frac{2\left(k^{3}+2 k^{2} M+2 k^{2} N+3 k M N+2 k+M+N\right)}{k(k+M)}\right. \\
& \times \delta_{\rho \bar{\sigma}} \delta_{j \bar{i}} J^{(\rho \bar{i})} J^{(\bar{\sigma} j)} \\
& +3 M J^{\alpha} J^{\alpha}-\frac{2\left(k^{2}-1\right)(2 k+M+N)}{k(k+M)} J^{\alpha} J_{f}^{\alpha} \\
& -\frac{2\left(k^{2}-1\right)(2 k+M+N)}{k(k+M)} \sqrt{\frac{M+N}{M N}} J^{u(1)} J_{f}^{u(1)} \\
& -\frac{2\left(k^{2}-1\right)(2 k+M+N)}{k(k+M)} J^{a} J_{f}^{a} \\
& +\frac{\left(k^{2}-1\right)(2 k+M+N)}{(k+M)} \delta_{\rho \bar{\sigma}} \delta_{j \bar{i}} \partial \psi^{(\rho \bar{i})} \psi^{(\bar{\sigma} j)} \\
& +\frac{3 N(k+N)}{(k+M)} J^{a} J^{a} \\
& -\frac{\left(k^{2}-1\right)(2 k+M+N)}{(k+M)} \delta_{\rho \bar{\sigma}} \delta_{j \bar{i}} \psi^{(\rho \bar{i})} \partial \psi^{(\bar{\sigma} j)} \\
& +\frac{3(k+N)(M+N)}{k} J^{u(1)} J^{u(1)}
\end{aligned}
$$

$\overline{{ }^{21} \text { If we act } G^{ \pm}}(z)$ on $T_{\text {boson }}(w)$ appearing in the footnote 9 , then we obtain the spin- $\frac{3}{2}$ current at the second order pole which is proportional to $G^{ \pm}(w)$. After acting on $G^{\mp}(z)$ further, then we obtain the second order pole which is proportional to $K(w)$ : the lowest component of (2.9). This is one way to observe that the multiplet of (2.9) is an $\mathcal{N}=2$ extension of the stress energy tensor in the bosonic coset model. 


$$
\begin{aligned}
& +\frac{N M\left(k^{3}+2 k^{2} M+2 k^{2} N+3 k M N+2 k+M+N\right)}{k(k+M)} \\
& \left.\sqrt{\frac{M+N}{M N}} \partial J^{u(1)}\right] .
\end{aligned}
$$

Note that the second, fifth and ninth terms in (4.7) do not arise in the stress energy tensor $T$ (2.5) and we cannot express this in terms of the known currents obtained so far. The fifth term is characteristic of this spin-2 current (or of modified stress energy tensor in Appendix A) in the sense that we do not see this term from the bosonic stress energy tensor also. In doing this computation, we need to obtain the OPE between $G^{-, a}(z)$ and $G^{+, b}(w)$ when we consider the OPE between the $G^{-}(z)$ and the second term of (4.5) at the coordinate $w$. See also Appendix D where the corresponding OPE is written in terms of the coset fields and Sect. 6 where the right hand side of this OPE is expressed in terms of the known currents explicitly.

In Sect. 7, we will calculate the OPE between this singlet spin-2 current and itself.

\subsection{Construction of second and third components}

From the Thielemans package [80] with [81], we can check that the OPE between $G^{+}(z)$ and $W^{-(2), 0}(w)$ does not contain the singular terms except the first order pole for fixed $N=5, M=4$ values. It turns out that the first order pole of this OPE is proportional to $G^{+\left(\frac{5}{2}\right), 0}(w)$. Then we should determine the coefficient appearing in front of this spin- $\frac{5}{2}$ current. As described before, we can focus on the particular nonderivative term of $G^{+\left(\frac{5}{2}\right), 0}(w)$. That is, the first term of (4.5). We can observe that the contribution from this term comes from the first three terms in $W^{-(2), 0}(w)$ of (4.7) and by collecting all the contributions we can obtain the final coefficient appearing in the spin- $\frac{5}{2}$ current $G^{+\left(\frac{5}{2}\right), 0}(w)$ in the above OPE. This implies that the spin- $\frac{5}{2}$ current $G^{+\left(\frac{5}{2}\right), 0}$ belongs to the second component of this $\mathcal{N}=2$ multiplet. See also Appendix B.

Similarly, we should also check whether the spin- $\frac{5}{2}$ current $G^{-\left(\frac{5}{2}\right), 0}$ belongs to the third component of this $\mathcal{N}=2$ multiplet or not. In this case, we also observe that the OPE between $G^{-}(z)$ and $W^{-(2), 0}(w)$ does not contain the singular terms except the first order pole for fixed $N=5, M=4$ values. By considering the first three terms in $W^{-(2), 0}(w)$ of (4.7) and collecting all the contributions, the final coefficient appearing in the spin- $\frac{5}{2}$ current $G^{-\left(\frac{5}{2}\right), 0}(w)$ in the above OPE can be fixed completely. Therefore, the spin- $\frac{5}{2}$ current $G^{-\left(\frac{5}{2}\right), 0}$ belongs to the third component of this $\mathcal{N}=2$ multiplet.

Then we have the following currents obtained so far in this multiplet

$\left(W^{-(2), 0}, G^{+\left(\frac{5}{2}\right), 0}, G^{-\left(\frac{5}{2}\right), 0}, ?\right)$, with the coset field contents given in (4.7), (4.5) and (4.6). The last component of (4.8) will be described in next subsection and we expect that the corresponding generalization of (4.1) will appear and further checks will be given later.

\subsection{Construction of last component}

In this subsection, we would like to construct the unknown last component in (4.8). First of all, in the previous description of (4.7), we have obtained the following result

$\left.G^{-}(z) G^{+\left(\frac{5}{2}\right), 0}(w)\right|_{\frac{1}{(z-w)^{2}}}=W^{-(2), 0}(w)$.

According to the explanation of Appendix B, the last component of the $\mathcal{N}=2$ multiplet can be obtained from the next first order pole in the OPE between $G^{-}(z)$ and $G^{+\left(\frac{5}{2}\right), 0}(w)$. After subtracting the descendant term associated with (4.9) in the first order pole, we arrive at the following relation

$$
\left.W^{+(3), 0}(w) \equiv G^{-}(z) G^{+\left(\frac{5}{2}\right), 0}(w)\right|_{\frac{1}{(z-w)}}-\frac{1}{4} \partial W^{-(2), 0}(w) .
$$

Then from the first order pole in the OPE between $G^{-}(z)$ and $G^{+\left(\frac{5}{2}\right), 0}(w)$ with derivative terms (4.10), we eventually obtain the following singlet spin-3 current as follows:

$$
\begin{aligned}
& W^{+(3), 0}=\frac{\left(k^{2}-4\right)(k+M+N)(3 k+2 M+2 N)}{k M(k+2 M)} b_{1} \\
& \times\left\{-3\left[t_{\sigma \bar{\sigma}}^{\alpha} \delta_{j \bar{i}} J^{(\bar{\sigma} j)} J^{\alpha} J^{(\sigma \bar{i})}\right.\right. \\
& -t_{\rho \bar{v}}^{\alpha} \delta_{m \bar{m}} t_{\sigma \bar{\sigma}}^{\alpha} \delta_{j \bar{i}} \psi^{(\bar{\sigma} j)}\left(\left(\psi^{(\rho \bar{m})} J^{(\bar{v} m)}\right) J^{(\sigma \bar{i})}\right) \\
& +\sqrt{\frac{M+N}{M N}} t_{\sigma \bar{\sigma}}^{\alpha} \delta_{j \bar{i}} \psi^{(\bar{\sigma} j)} J^{\alpha} \psi^{(\sigma \bar{i})} J^{u(1)} \\
& -\left(t^{\beta} t^{\alpha}\right)_{\rho \bar{\sigma}} \delta_{j \bar{i}} J^{\alpha} J^{\beta} \psi^{(\rho \bar{i})} \psi^{(\bar{\sigma} j)} \\
& +t_{i \bar{k}}^{a} t_{\sigma \bar{\sigma}}^{\alpha} J^{\alpha} J^{a} \psi^{(\sigma \bar{k})} \psi^{(\bar{\sigma} i)} \\
& \left.-k t_{\sigma \bar{\sigma}}^{\alpha} \delta_{j \bar{i}} \psi^{(\bar{\sigma} j)} J^{\alpha} \partial \psi^{(\sigma \bar{i})}\right] \\
& -\frac{3}{(k+M)}(k+N) \\
& \times\left[\frac{1}{2}\left(-N J^{a} \partial J^{a}+k J^{a} \partial J_{f}^{a}\right)\right. \\
& \left.+G^{-, a} G^{+, a}+J^{a} W^{+(2), a}+i f^{a b c} J^{a} J^{b} J_{f}^{c}\right] \\
& -\frac{3}{k} \sqrt{\frac{M+N}{M N}}(k+N)\left[\sqrt{\frac{M+N}{M N}} G^{-} G^{+}\right. \\
& -\frac{1}{2}(k+M+N) J^{u(1)} \partial K
\end{aligned}
$$




$$
\begin{aligned}
& \left.+(k+M+N) J^{u(1)} T-\frac{1}{2} J^{u(1)}\left(J^{a}+J_{f}^{a}\right)^{2}\right] \\
& +3(k+N)\left[\delta_{\rho \bar{\sigma}} \delta_{j \bar{i}} J^{(\bar{\sigma} j)} \partial J^{(\rho \bar{i})}\right. \\
& +\sqrt{\frac{M+N}{M N}} \delta_{\rho \bar{\sigma}} \delta_{j \bar{i}} \psi^{(\bar{\sigma} j)} \partial\left(\psi^{(\rho \bar{i})} J^{u(1)}\right) \\
& +t_{\rho \bar{\sigma}}^{\alpha} \delta_{j \bar{i}} \psi^{(\bar{\sigma} j)} \partial\left(\psi^{(\rho \bar{i})} J^{\alpha}\right) \\
& -t_{i \bar{k}}^{a} \delta^{i \bar{i}} \delta_{\rho \bar{\sigma}} \delta_{j \bar{i}} \psi^{(\bar{\sigma} j)} \partial\left(\psi^{(\rho \bar{k})} J^{a}\right) \\
& \left.-k \delta_{\rho \bar{\sigma}} \delta_{j \bar{i}} \psi^{(\bar{\sigma} j)} \partial^{2} \psi^{(\rho \bar{i})}\right] \\
& -\frac{\left(k^{2}-1\right)(2 k+M+N)}{k(k+M)} \\
& \times\left[-\frac{1}{2}(k+M+N) \partial^{2} K\right. \\
& +(k+M+N) \partial T \\
& \left.\left.-\frac{1}{2} \partial\left(J^{a}+J_{f}^{a}\right)^{2}\right]\right\}-\frac{1}{4} \partial W^{-(2), 0} .
\end{aligned}
$$

We do not simplify this expression further because there are not too many common terms. The second term can be further simplified by using the rearrangement lemma in [78]. Except the last term of (4.11), we can read off the five contributions denoted by each two brackets inside of curly bracket from (4.5). Note that the first term of (4.11) originates from the $b_{6}$ term of (4.1). ${ }^{22}$ We can understand this feature from the OPE between $G^{-}(z)$ and the first term of $G^{+\left(\frac{5}{2}\right), 0}(w)$ where the OPE between the former and the spin- $\frac{1}{2}$ operator provides the spin-1 current transforming as $(\overline{\mathbf{N}}, \mathbf{M})$. In this sense, the singlet spin-3 current (4.11) is a generalization of the spin-3 current (4.1) in the $\mathcal{N}=2$ supersymmetric coset model. As mentioned before, some OPEs in Appendix D (or in Sect. 6) can be used.

Then we have the following currents of this multiplet we are considering in this section

$\left(W^{-(2), 0}, G^{+\left(\frac{5}{2}\right), 0}, G^{-\left(\frac{5}{2}\right), 0}, W^{+(3), 0}\right)$.

They also satisfy the regular conditions as in (4.3). It would be interesting to construct the lowest singlet spin- 2 current from the beginning directly without using the information of the spin-3 current in the bosonic coset model and by collecting all the possible composite singlet spin-2 operators with arbitrary coefficients. The nontrivial part in this direction is how to fix these coefficients which will depend on the three parameters $k, N$ and $M$ explicitly.

In next subsection, in order to check that this multiplet (4.12) is right $\mathcal{N}=2$ multiplet, other OPEs with the generators of $\mathcal{N}=2$ superconformal algebra are described. ${ }^{23}$

4.4 The OPEs with the currents of $\mathcal{N}=2$ superconformal algebra

The OPEs between the spin-1 current of (2.9) and the currents of (4.12), by realizing the corresponding OPEs for fixed $N$ and $M$, can be summarized by

$$
\begin{aligned}
& K(z) W^{-(2), 0}(w)=\frac{1}{(z-w)^{2}}\left[\frac{2\left(k^{2}-1\right)\left(k^{2}-4\right)}{k^{2} M(k+M)(k+2 M)} \times(k+M+N)^{2}(2 k+M+N)(3 k+2 M+2 N) b_{1}\right] \\
& K(w)+\cdots, K(z) G^{ \pm\left(\frac{5}{2}\right), 0}(w)=\mp \frac{1}{(z-w)^{2}}\left[\frac{\left(k^{2}-1\right)\left(k^{2}-4\right)}{k^{2} M(k+M)(k+2 M)}\right. \\
& \left.\times(k+M+N)(2 k+M+N)(3 k+2 M+2 N) b_{1}\right] G^{ \pm}(w) \\
& \pm \frac{1}{(z-w)} G^{ \pm\left(\frac{5}{2}\right), 0}(w)+\cdots, K(z) W^{+(3), 0}(w)=\frac{1}{(z-w)^{2}}\left[-W^{-(2), 0}\right. \\
& -\frac{\left(k^{2}-1\right)\left(k^{2}-4\right)(k+M+N)^{2}(2 k+M+N)(3 k+2 M+2 N)}{k^{2} M(k+M)(k+2 M)} b_{1} \\
& \left.\times\left(T-\frac{1}{2(k+M+N)}\left(J^{a}+J_{f}^{a}\right)^{2}\right)\right](w)+\cdots .
\end{aligned}
$$

\footnotetext{
22 For convenience, we present, as in the footnote 13, some relevant OPEs $J^{a}(z) G^{ \pm}(w)= \pm \frac{1}{(z-w)} G^{ \pm, a}(w)+\cdots, J^{\alpha}(z) G^{+}(w)=$ $\frac{1}{(z-w)} t_{\rho \bar{\sigma}}^{\alpha} \delta_{j \bar{k}} \psi^{(\bar{\sigma} j)} J^{(\rho \bar{k})}(w)+\cdots$ and $J^{\alpha}(z) G^{-}(w)=-\frac{1}{(z-w)}$ $t_{\rho \bar{\sigma}}^{\alpha} \delta_{j \bar{k}} \psi^{(\rho \bar{k})} J^{(\bar{\sigma} j)}(w)+\cdots$. These OPEs will be used at various places of this paper.
}

\footnotetext{
${ }^{23}$ Compared with the bosonic coset model description, the last component of (4.12) is an $\mathcal{N}=2$ supersymmetric version of previous spin-3 current (4.1) and the remaining three components are its superpartners. In (2.9), the stress energy tensor (2.5) is the $\mathcal{N}=2$ supersymmetric version of $T_{\text {boson }}$ of the footnote 9 . There are also three other components in (2.9). It seems that there is no bosonic analog for the first $\mathcal{N}=2$ multiplet of (3.3).
} 
Note that in the last OPE of (4.13), the combination of stress energy tensor with $\left(J^{a}+J_{f}^{a}\right)^{2}$ term is exactly the same as the one in the OPE between the supersymmetry generators in Appendix A. Compared with the ones in Appendix B, there are additional terms in the right hand sides of the OPEs. Although we can check all the relevant terms in the right hand sides, the structure constants appearing in the composite operators of the right hand sides in (4.13) can be determined by focusing on the particular operators as before.

For example, in the last OPE of (4.13), we can focus on the $J^{\alpha} J^{\alpha}$ term in the second order pole, which determines the coefficient of $W^{-(2), 0}$ because we do not see this particular term in the remaining two terms. After that by considering the $J^{a} J_{f}^{a}$ term, which will appear in the first and the last terms of the second order pole, we can fix the coefficient of
$\mathcal{N}=2$ primary conditions described in Appendix B. In the third OPE of (4.14), we can also obtain the singlet spin-3 current by using the upper sign. The lower sign was used in previous analysis. In the last OPE of (4.14), we can fix the structure constant of the second order pole (where there are no descendant terms) by focusing on the first terms in (4.5) or (4.6). In other words, in the computation of the left hand side of these OPEs, after we select the possible terms from $W^{+(3), 0}$ (4.11) for fixed $(N, M)$ in the Thielemans package and add all the contributions from those terms for generic $(N, M)$, we compute them manually and obtain $k, N$ and $M$ dependence explicitly. It turns out that the first order pole is given by the descendant terms only.

Finally, we can compute the OPEs with stress energy tensor. It turns out that

$$
\begin{aligned}
& T(z) W^{-(2), 0}(w)=\frac{1}{(z-w)^{2}} 2 W^{-(2), 0}(w)+\frac{1}{(z-w)} \partial W^{-(2), 0}(w)+\cdots \\
& T(z) G^{ \pm\left(\frac{5}{2}\right), 0}(w)=\frac{1}{(z-w)^{2}} \frac{5}{2} G^{ \pm\left(\frac{5}{2}\right), 0}(w)+\frac{1}{(z-w)} \partial G^{ \pm\left(\frac{5}{2}\right), 0}(w)+\cdots \\
& T(z) W^{+(3), 0}(w)=-\frac{1}{(z-w)^{4}} \frac{3\left(k^{2}-1\right)\left(k^{2}-4\right)(3 k+2 M+2 N)(k+M+N)^{2}(2 k+M+N)}{2 k^{2} M(k+M)(k+2 M)} b_{1} K(w) \\
& +\frac{1}{(z-w)^{2}} 3 W^{+(3), 0}(w)+\frac{1}{(z-w)} \partial W^{+(3), 0}(w)+\cdots
\end{aligned}
$$

$\left(J^{a}+J_{f}^{a}\right)^{2}$ term because the coefficient of the first term is already known from the previous analysis. Finally, from the $J^{a} J^{a}$ term in the second order pole, which will appear in the second and the last terms, the coefficient appearing in the stress energy tensor (the second term) can be obtained because the coefficient of the last terms is known.

Next we summarize the following OPEs between the spin$\frac{3}{2}$ currents of the $\mathcal{N}=2$ superconformal algebra and the currents of $\mathcal{N}=2$ multiplet in this section
The singlet spin-3 current $W^{+(3), 0}$ in (4.15) is a quasi primary current under the stress energy tensor. If we add the extra term

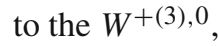

$$
W^{+(3), 0}+\frac{3}{4} K W^{-(2), 0}
$$

then we can remove the fourth order pole in the last OPE of (4.15) with stress energy tensor because the OPE between $K(z)$ and $W^{-(2), 0}(w)$ has only a second order pole from

$$
\begin{aligned}
& G^{ \pm}(z) W^{-(2), 0}(w)=\frac{1}{(z-w)} 2(k+M+N) G^{ \pm\left(\frac{5}{2}\right), 0}(w)+\cdots, \\
& G^{ \pm}(z) G^{ \pm\left(\frac{5}{2}\right), 0}(w)=0+\cdots, G^{ \pm}(z) G^{\mp\left(\frac{5}{2}\right), 0}(w)= \\
& \quad \pm \frac{1}{(z-w)^{3}} \frac{\left(k^{2}-1\right)\left(k^{2}-4\right)(k+M+N)^{2}(2 k+M+N)(3 k+2 M+2 N)}{k^{2} M(k+M)(k+2 M)} b_{1} K(w) \\
& \quad+\frac{1}{(z-w)^{2}} W^{-(2), 0}(w)+\frac{1}{(z-w)}\left[\frac{1}{4} \partial W^{-(2), 0} \mp W^{+(3), 0}\right](w)+\cdots, \\
& G^{ \pm}(z) W^{+(3), 0}(w)=\mp \frac{1}{(z-w)^{3}} \frac{\left(k^{2}-1\right)\left(k^{2}-4\right)(k+M+N)^{2}(2 k+M+N)(3 k+2 M+2 N)}{k_{1} G^{ \pm}(w)} \\
& \quad \pm \frac{1}{(z-w)^{2}} \frac{5}{2}(k+M+N) G^{ \pm\left(\frac{5}{2}\right), 0}(w) \pm \frac{1}{(z-w)} \frac{1}{5} \frac{5}{2}(k+M+N) \partial G^{ \pm\left(\frac{5}{2}\right), 0}(w)+\cdots
\end{aligned}
$$

As before, there are additional higher order terms in the right hand sides of these OPEs when we compare with the standard 
(4.13). Then (4.16) becomes a primary field. We can easily obtain the OPEs between the currents of $\mathcal{N}=2$ superconformal algebra and the composite operator $K W^{-(2), 0}$ and then we will obtain the corresponding OPEs containing (4.16). ${ }^{24}$

Therefore, the singlet currents are given by (4.7), (4.5), (4.6) and (4.11) in the coset realization and they satisfy (4.13), (4.14), and (4.15) under the action of the generators of $\mathcal{N}=2$ superconformal algebra. The right hand sides of these OPEs contain the currents of $\mathcal{N}=2$ multiplet, the composite spin2 operator $\left(J^{a}+J_{f}^{a}\right)^{2}$ nonlinearly as well as the currents of $\mathcal{N}=2$ superconformal algebra. We will describe some features on the OPEs between this $\mathcal{N}=2$ multiplet and itself in Sect. 7.

\section{The nonsinglet multiplet of spins $\left(2, \frac{5}{2}, \frac{5}{2}, 3\right)$}

We continue to construct the $\mathcal{N}=2$ multiplet which has $S U(M)$ index $a$. From the experience of [12], we realize that the OPE between the spin-2 current $K^{a}(z)$ and itself $K^{b}(w)$ produces the spin-3 current $P^{c}(w)$. We can start with either this spin-2 current plus other terms as a candidate for the lowest component or this spin-3 current plus other terms as a candidate for the last component of the $\mathcal{N}=2$ multiplet. Let us take the latter.

\subsection{Construction of lowest component}

Let us consider the spin-3 current found in bosonic coset model of [3]

$$
\begin{aligned}
P^{a}= & a_{1} t_{\rho \bar{\sigma}}^{\alpha} t_{j \bar{i}}^{a} J^{\alpha} J^{(\rho \bar{i})} J^{(\bar{\sigma} j)} \\
& +a_{2} J^{\alpha} J^{\alpha} J^{a}+a_{3} J^{b} J^{b} J^{a}+a_{4} J^{a} J^{u(1)} J^{u(1)} \\
& +a_{5} d^{a b c} \delta_{\rho \bar{\rho}} t_{j \bar{i}}^{b} J^{c}\left(J^{(\rho \bar{i})} J^{(\bar{\rho} j)}+J^{(\bar{\rho} j)} J^{(\rho \bar{i})}\right) \\
& +a_{7} \delta_{\rho \bar{\sigma}} t_{j \bar{i}}^{a} J^{u(1)}\left(J^{(\rho \bar{i})} J^{(\bar{\sigma} j)}+J^{(\bar{\sigma} j)} J^{(\rho \bar{i})}\right) \\
& +a_{8} \delta_{\rho \bar{\sigma}} \delta_{j \bar{i}} J^{a}\left(J^{(\rho \bar{i})} J^{(\bar{\sigma} j)}+J^{(\bar{\sigma} j)} J^{(\rho \bar{i})}\right) \\
& +a_{9} d^{a b c} J^{b} J^{c} J^{u(1)}+a_{11} i f^{a b c} \partial J^{b} J^{c} \\
& +a_{12} \delta_{\rho \bar{\sigma}} t_{j \bar{i}}^{a} \partial J^{(\rho \bar{i})} J^{(\bar{\sigma} j)} \\
& +a_{13} \delta_{\rho \bar{\sigma}} t_{j \bar{i}}^{a} \partial J^{(\bar{\sigma} j)} J^{(\rho \bar{i})}(z)+a_{16} \partial^{2} J^{a} \\
& +a_{17} 6 \operatorname{Tr}\left(t^{a} t^{(b} t^{c} t^{d)}\right) J^{b} J^{c} J^{d} .
\end{aligned}
$$

\footnotetext{
${ }^{24}$ In the OPE between $K(z)$ and $K W^{-(2), 0}(w)$, the second order pole has $W^{-(2), 0}$ and $K K$ terms. Similarly, the OPE between $G^{+}(z)$ and $K W^{-(2), 0}(w)$ provides the second order pole with $G^{+\left(\frac{5}{2}\right), 0}$ and Footnote 28 the first order pole together with $G^{+} W^{-(2), 0}$ and $K G^{+\left(\frac{5}{2}\right), 0}$. Moreover, the OPE between $G^{-}(z)$ and $K W^{-(2), 0}(w)$ leads to the second order pole with $G^{-\left(\frac{5}{2}\right), 0}$ and the first order pole together with $G^{-} W^{-(2), 0}$ and $K G^{-\left(\frac{5}{2}\right), 0}$. We observe that there are several nonlinear terms.
}

Here the relative coefficients depend on $k, N$ and $M$ as follows:

$$
\begin{aligned}
a_{2} & =\frac{a_{1}}{k}, \quad a_{3}=\frac{N(k+2 N)}{k(k+M)(3 k+2 M)} a_{1}, \\
a_{4} & =\frac{(k+2 N)(M+N)}{k^{2} M} a_{1}, \\
a_{5} & =-\frac{(k+2 N)}{4(k+M)} a_{1}, \quad a_{7}=\frac{(k+2 N)}{2 k} \sqrt{\frac{M+N}{M N}} a_{1}, \\
a_{8} & =-\frac{(k+2 N)}{2 k M} a_{1}, \\
a_{9} & =-\frac{(k+2 N) N}{2 k(k+M)} \sqrt{\frac{(M+N)}{M N}} a_{1}, \\
a_{11} & =\frac{\left(k^{2}-8\right) N(k+2 N)}{4 k(k+M)(3 k+2 M)} a_{1}, \\
a_{12} & =-\frac{1}{2}(k+2 N) a_{1}, \\
a_{13} & =\frac{1}{2}(k+2 N) a_{1}, \\
a_{16} & =-\frac{N\left(6 k^{3}+9 k^{2} M+4 k M^{2}+12 M\right)(k+2 N)}{12 k(k+M)(3 k+2 M)} a_{1}, \\
a_{17} & =\frac{N(k+2 N)}{6(k+M)(3 k+2 M)} a_{1} .
\end{aligned}
$$

First of all this nonsinglet spin-3 current satisfies

$$
\begin{aligned}
& P^{a}(z)\left(J^{u(1)}+\sqrt{\frac{M+N}{M N}} J_{f}^{u(1)}\right)(w) 0+\cdots, \\
& =P^{a}(z)\left(J^{\alpha}+J_{f}^{\alpha}\right)(w)=0+\cdots .
\end{aligned}
$$

That is, the regular conditions in the bosonic coset model are valid in the $\mathcal{N}=2$ supersymmetric coset model because the additional terms in (5.3) come from the complex fermions which commute with the spin-3 currents made by bosonic operators purely.

Let us describe the OPEs between the supersymmetry generators of the $\mathcal{N}=2$ superconformal algebra and the nonsinglet spin-3 current of the bosonic coset model. We use the OPEs between the spin- 1 currents and the spin- 3 current described in Appendix C. It turns out that with the help of (5.1) and (5.2)

$$
\begin{aligned}
G^{ \pm} & (z) P^{a}(w) \\
= & \mp \frac{1}{(z-w)^{3}} \frac{\left(k^{2}-1\right)\left(k^{2}-4\right)(2 k+M+N)(3 k+2 M+2 N)}{2 k^{2}(k+M)(3 k+2 M)} \\
& \times b_{1} G^{ \pm, a}(w) \\
\pm & \frac{1}{(z-w)^{2}} \hat{V}^{ \pm\left(\frac{5}{2}\right), a}(w)+\mathcal{O}\left(\frac{1}{(z-w)}\right) .
\end{aligned}
$$

The point here is that the second order pole of (5.4) possesses exactly the first, second and fifth terms of $V^{+\left(\frac{5}{2}\right), a}$ (3.10) or $V^{-\left(\frac{5}{2}\right), a}$ (3.12) with same relative coefficients. Then this implies that we can express the above second order pole of (5.4) in terms of previous known operators. We can absorb 
those three unwanted terms by using $V^{ \pm\left(\frac{5}{2}\right), a}$ and recombine the remaining terms with the known operators appearing in the second order pole.

Then we obtain the following spin- $\frac{5}{2}$ currents which are not new because they are previously known expressions

$$
\begin{aligned}
\hat{V}^{ \pm\left(\frac{5}{2}\right), a}= & \frac{\left(k^{2}-4\right)(3 k+2 M+2 N)}{2 k(k+M)} a_{1}\left[\frac{1}{2} V^{ \pm\left(\frac{5}{2}\right), a}\right. \\
& \pm \frac{(2 k+M+N)}{k(3 k+2 M)} J^{a} G^{ \pm} \\
& +\frac{\left(k^{2}-1\right) M(2 k+M+N)}{3 k(2 k+M)(3 k+2 M)} \partial G^{ \pm, a} \\
& +\frac{(2 k+M+N)}{2(3 k+2 M)} i f^{a b c} J^{b} G^{ \pm, c} \\
& \left.\mp \frac{M(2 k+M+N)}{2(2 k+M)(3 k+2 M)} d^{a b c} J^{b} G^{ \pm, c}\right],
\end{aligned}
$$

where $V^{ \pm\left(\frac{5}{2}\right), a}$ are given by (3.10) and (3.12). The last four terms in $\hat{V}^{ \pm,\left(\frac{5}{2}\right), a}(5.5)$ are primary operators under the stress energy tensor $T$ (2.5) and they can be seen from (3.10) and (3.12).

As done in previous section, the second order pole in the OPEs of $G^{\mp}(z) \hat{V}^{ \pm,\left(\frac{5}{2}\right), a}(w)$ leads to the following primary nonsinglet spin-2 current

$\left.G^{\mp}(z) \hat{V}^{ \pm,\left(\frac{5}{2}\right), a}(w)\right|_{\frac{1}{(z-w)^{2}}}= \pm W^{-(2), a}(w)$,

where the lowest nonsinglet spin-2 current can be written as

$$
\begin{aligned}
W^{-(2), a}= & \frac{\left(k^{2}-4\right)(3 k+2 M+2 N)}{k(k+M)(3 k+2 M)} a_{1} \\
& \times\left[\frac{1}{4}(2 k+M)(3 k+M+3 N) K^{a}\right. \\
& +\frac{1}{k}\left(k^{2}-1\right)(2 k+M+N)\left(W^{+(2), a}\right. \\
& \left.\left.+\frac{1}{2} i f^{a b c} J^{b} J_{f}^{c}\right)\right] .
\end{aligned}
$$

Here we have the relations $W^{+(2), a}(3.1)$ and $K^{a}$ (3.2) which are given by the coset fields. Note that this is a new quantity which cannot be written in terms of the previous known currents obtained so far. The spin-2 current $K^{a}$ obtained in the bosonic coset model is not an element of any $\mathcal{N}=2$ multiplet. When we compute the OPEs between the supersymmetry generators and the fourth or fifth terms of (5.5) in the computation of (5.6), we should use some property appearing in Appendix D or Sect. 6 together with the footnote 22. Note that this (5.7) is a new current in the sense that although it contains both the known current $W^{+(2), a}$ (3.1) and known operator $i f^{a b c} J^{b} J_{f}^{c}$ which is primary, it also contains the spin-2 current $K^{a}$ (3.2). ${ }^{25}$ As soon as the spin-2 current $K^{a}$ appears in any OPEs we are considering, then we should replace it with other two currents $W^{ \pm(2), a}$ and other terms by using the relation (5.7).

Therefore, we have obtained the nonsinglet spin- 2 current in (5.7) by acting the supersymmetry generators of the $\mathcal{N}=2$ superconformal algebra on the spin- 3 current successively. In next subsections, we would like to construct its superpartners explicitly.

\subsection{Construction of second and third components}

Because we have found the lowest component of the $\mathcal{N}=2$ multiplet we consider in this section, it is straightforward to determine the other three components. For given the lowest component in (5.7), we calculate the following OPEs

$G^{ \pm}(z) W^{-(2), a}(w)=\frac{1}{(z-w)} G^{ \pm\left(\frac{5}{2}\right), a}(w)+\cdots$.

It is a good sign for the existence of the pole one term by realizing Appendix B. When we compute the above OPE for the first term of (5.7), we can use the previous relations in (3.9) and (3.11). For the second term of (5.7), we can use the relations (3.5) and (3.6). For the third term of (5.7), we obtain the composite operators.

After simplifying further, the first order pole provides the following primary nonsinglet spin- $\frac{5}{2}$ currents

$$
\begin{aligned}
G^{ \pm\left(\frac{5}{2}\right), a}= & \frac{\left(k^{2}-4\right)(3 k+2 M+2 N)}{k(k+M)(3 k+2 M)} a_{1} \\
& \times\left[\frac{1}{4}(2 k+M)(3 k+M+3 N) V^{ \pm\left(\frac{5}{2}\right), a}\right. \\
& -\frac{1}{2 k}\left(k^{2}-1\right)(2 k+M+N) i f^{a b c} G^{ \pm, b} J_{f}^{c} \\
& +\frac{1}{2 k}\left(k^{2}-1\right)(2 k+M+N) i f^{a b c} J^{b} G^{ \pm, c} \\
& \left.-\frac{1}{6 k}\left(k^{2}-1\right) M(2 k+M+N) \partial G^{ \pm, a}\right] .
\end{aligned}
$$

Due to the presence of the third term of (5.7), we have the second term of (5.9) which does not appear in (5.5). We can check that the three terms of (5.9) except the first one are given by the known operators and are primary under the stress energy tensor. Because the first term in (5.9) cannot be written in terms of the known currents, we have the new primary spin- $\frac{5}{2}$ currents. Note that in the OPE of (5.8), there are no higher order pole terms which can be checked for fixed $N$ and $M$ also.

25 That is $K^{a}$ is one of the currents in the bosonic coset model but this is not an element of any $\mathcal{N}=2$ multiplet in the $\mathcal{N}=2$ supersymmetric coset model. 


\subsection{Construction of last component}

As done in previous section, we construct the last component of this multiplet. We can compute the following OPE

$$
\left.G^{-}(z) G^{+\left(\frac{5}{2}\right), a}(w)\right|_{\frac{1}{(z-w)^{2}}}=(2 k+M+2 N) W^{-(2), a}(w) .
$$

The structure constant of (5.10) can be determined by keeping track of the particular term in the nonsinglet spin-2 current $W^{-(2), a}(w)$.

Let us introduce the final last component which is given by

$$
\begin{aligned}
& \left.W^{+(3), a} \equiv G^{-}(z) G^{+\left(\frac{5}{2}\right), a}(w)\right|_{\frac{1}{(z-w)}} \\
& -\frac{1}{4} \partial\left(\left.G^{-}(z) G^{+\left(\frac{5}{2}\right), a}(w)\right|_{\frac{1}{(z-w)^{2}}}\right) \\
& =\frac{\left(k^{2}-4\right)(3 k+2 M+2 N)}{k(k+M)(3 k+2 M)} a_{1} \\
& \times\left\{\frac{1}{4}(2 k+M)(3 k+M+3 N)\right. \\
& \times\left(-2(k+N)\left[\delta_{\rho \sigma} t_{j \bar{i}}^{a} J^{(\bar{\sigma} j)} \partial J^{(\rho \bar{i})}\right.\right. \\
& -k \delta_{\rho \bar{\sigma}} t_{j \bar{i}}^{a} \psi^{(\bar{\sigma} j)} \partial^{2} \psi^{(\rho \bar{i})} \\
& +\sqrt{\frac{M+N}{M N}} \delta_{\rho \sigma} t_{j \bar{i}}^{a} \psi^{(\bar{\sigma} j)} \partial\left(\psi^{(\rho \bar{i})} J^{u(1)}\right) \\
& +\delta_{\rho \bar{\sigma}} t_{j \bar{i}}^{a} t_{\sigma \bar{\rho}}^{\alpha} \delta^{\rho \bar{\rho}} \psi^{(\bar{\sigma} j)} \partial\left(\psi^{(\sigma \bar{i})} J^{\alpha}\right) \\
& \left.-\delta_{\rho \bar{\sigma}}\left(t^{a} t^{b}\right)_{j \bar{k}} \psi^{(\bar{\sigma} j)} \partial\left(\psi^{(\rho \bar{k})} J^{b}\right)\right] \\
& -\frac{2}{k}(k+N) \sqrt{\frac{M+N}{M N}}\left[\sqrt{\frac{M+N}{M N}} G^{-} G^{+, a}\right. \\
& -\frac{N}{2} J^{u(1)} \partial J^{a}+\frac{k}{2} J^{u(1)} \partial J_{f}^{a} \\
& \left.+J^{u(1)} W^{+(2), a}+i f^{a b c} J^{u(1)} J^{b} J_{f}^{c}\right] \\
& -\frac{2}{k M}(k+M+N)\left[G^{-, a} G^{+}\right. \\
& -\frac{1}{2}(k+M+N) J^{a} \partial K+(k+M+N) J^{a} T \\
& \left.-\frac{1}{2} J^{a}\left(J^{b}+J_{f}^{b}\right)^{2}\right]-\left[i f^{a b c} G^{-, b} G^{+, c}\right. \\
& +\frac{1}{2}\left(-N J^{b} \partial J^{c}+k J^{b} \partial J_{f}^{c}\right) i f^{a b c} \\
& \left.+i f^{a b c} J^{b} W^{+(2), c}-f^{a b c} f^{c d e} J^{b} J^{d} J_{f}^{e}\right]
\end{aligned}
$$

$$
\begin{aligned}
& +\frac{(2 k+M+2 N)}{(2 k+M)}\left[d^{a b c} G^{-, b} G^{+, c}\right. \\
& -\frac{N}{2} d^{a b c} J^{b} \partial J^{c} \\
& +\frac{k}{2} d^{a b c} J^{b} \partial J_{f}^{c}+d^{a b c} J^{b} W^{+(2), c} \\
& \left.+d^{a b c} i f^{c d e} J^{b} J^{d} J_{f}^{e}\right] \\
& +2\left[t_{\rho \bar{\sigma}}^{\alpha} t_{j \bar{i}}^{a} J^{(\bar{\sigma} j)} J^{\alpha} J^{(\rho \bar{i})}\right. \\
& -t_{\rho \bar{\sigma}}^{\alpha} t_{j \bar{i}}^{a} t_{\rho_{1} \bar{v}}^{\alpha} \delta_{m \bar{m}} \psi^{(\bar{\sigma} j)}\left(\left(\psi^{\left(\rho_{1} \bar{m}\right)} J^{(\bar{v} m)}\right) J^{(\rho \bar{i})}\right) \\
& -k t_{\rho \bar{\sigma}}^{\alpha} t_{j \bar{i}}^{a} \psi^{(\bar{\sigma} j)} J^{\alpha} \partial \psi^{(\rho \bar{i})} \\
& +\sqrt{\frac{M+N}{M N}} t_{\rho \bar{\sigma}}^{\alpha} t_{j \bar{i}}^{a} \psi^{(\bar{\sigma} j)} J^{\alpha} \psi^{(\rho \bar{i})} J^{u(1)} \\
& +\left(t^{\beta} t^{\alpha}\right)_{\sigma \bar{\sigma}} t_{j \bar{i}}^{a} \psi^{(\bar{\sigma} j)} J^{\alpha} \psi^{(\sigma \bar{i})} J^{\beta} \\
& \left.-t_{\rho \bar{\sigma}}^{\alpha}\left(t^{a} t^{b}\right)_{j \bar{k}} \psi^{(\bar{\sigma} j)} J^{\alpha} \psi^{(\rho \bar{k})} J^{b}\right] \\
& -\frac{2}{3} \frac{2\left(k^{2}-1\right)(2 k+M+N)}{k(2 k+M)}\left[-\frac{N}{2} \partial^{2} J^{a}\right. \\
& \left.\left.+\frac{k}{2} \partial^{2} J_{f}^{a}+\partial W^{+(2), a}+i f^{a b c} \partial\left(J^{b} J_{f}^{c}\right)\right]\right) \\
& -\frac{1}{2 k}\left(k^{2}-1\right)(2 k+M+N) i f^{a b c} \\
& \times\left(-\frac{N}{2} \partial J^{b} J_{f}^{c}+\frac{k}{2} \partial J_{f}^{b} J_{f}^{c}+W^{+(2), b} J_{f}^{c}\right. \\
& \left.+i f^{b c d}\left(\left(J^{c} J_{f}^{d}\right) J_{f}^{c}\right)+G^{+, b} G^{-, c}\right) \\
& +\frac{1}{2 k}\left(k^{2}-1\right)(2 k+M+N)\left(i f^{a b c} G^{-, b} G^{+, c}\right. \\
& +\frac{1}{2}\left(-N J^{b} \partial J^{c}+k J^{b} \partial J_{f}^{c}\right) i f^{a b c} \\
& \left.+i f^{a b c} J^{b} W^{+(2), c}-f^{a b c} f^{c d e} J^{b} J^{d} J_{f}^{e}\right) \\
& -\frac{1}{6 k}\left(k^{2}-1\right) M(2 k+M+N)\left(-\frac{N}{2} \partial^{2} J^{a}\right. \\
& \left.\left.+\frac{k}{2} \partial^{2} J_{f}^{a}+\partial W^{+(2), a}+i f^{a b c} \partial\left(J^{b} J_{f}^{c}\right)\right)\right\} \\
& -\frac{1}{4}(2 k+M+2 N) \partial W^{-(2), a} \text {. }
\end{aligned}
$$

The first seventeen lines of (5.11) come from the spin- $\frac{5}{2}$ current $V^{+\left(\frac{5}{2}\right), a}$ consisting of seven independent terms in (3.10) and the remaining six lines come from the remaining three terms of (5.9). Then we can read off the corresponding OPEs between the spin- $\frac{3}{2}$ current and the spin- $\frac{5}{2}$ current by look- 
ing at the seven pairs of brackets inside the curly bracket. We observe that the $t_{\rho \bar{\sigma}}^{\alpha} t_{j \bar{i}}^{a} J^{(\bar{\sigma} j)} J^{\alpha} J^{(\rho \bar{i})}$ term appearing inside of the sixth pair of bracket originates from the $a_{1}$ term of (5.1) and therefore the above nonsinglet spin-3 current is a generalization of (5.1) in the $\mathcal{N}=2$ supersymmetric coset
5.4 The OPEs with the currents of $\mathcal{N}=2$ superconformal algebra

Let us present the OPEs with the spin- 1 current of the $\mathcal{N}=2$ superconformal algebra

$$
\begin{aligned}
& K(z) W^{-(2), a}(w)=\frac{1}{(z-w)^{2}} \frac{\left(k^{2}-1\right)\left(k^{2}-4\right)(3 k+2 M+2 N)(2 k+M+N)}{k^{2}(k+M)(3 k+2 M)} a_{1} \\
& \quad \times\left[N J^{a}-k J_{f}^{a}\right](w)+\cdots, K(z) G^{ \pm\left(\frac{5}{2}\right), a}(w) \\
& =\mp \frac{1}{(z-w)^{2}} \frac{\left(k^{2}-1\right)\left(k^{2}-4\right)(k+N)(2 k+M+N)(3 k+2 M+2 N)}{k^{2}(k+M)(3 k+2 M)} a_{1} G^{ \pm, a}(w) \pm \frac{1}{(z-w)} G^{ \pm\left(\frac{5}{2}\right), a}(w)+\cdots, \\
& \left.K(z) W^{+(3), a}(w)=\frac{1}{(z-w)^{3}}\left[\frac{\left(k^{2}-1\right)\left(k^{2}-4\right) M(2 k+M+N)(3 k+2 M+2 N)}{2 k^{2}(k+M)(3 k+2 M)} a_{1}\left(N J^{a}-k J_{f}^{a}\right)\right](w)\right]\left(w^{2}-1\right)\left(k^{2}-4\right)(k+N)(2 k+M+N)(3 k+2 M+2 N) \\
& \quad+\frac{1}{(z-w)^{2}}\left[-\frac{1}{2} \partial(k+M)(3 k+2 M)\right. \\
& \left.\quad \times a_{1} i f^{a b c} J^{b} J_{f}^{c}-\frac{\left(k^{2}-1\right)\left(k^{2}-4\right)(k+N)(2 k+M+N)(3 k+2 M+2 N)}{k^{2}(k+M)(3 k+2 M)} a_{1} W^{+2(a)}\right](w)+\cdots
\end{aligned}
$$

model we are describing in this paper. As before, in the OPE between $G^{-}(z)$ and the sixth term of $V^{+\left(\frac{5}{2}\right), a}(w)$, the OPE between $G^{-}$and the spin- $\frac{1}{2}$ operator leads to the spin-1 current transforming as $(\overline{\mathbf{N}}, \mathbf{M})$. By combining this with the two other spin-2 currents, we obtain the first term inside the sixth pair of brackets.

Then we are left with the following currents

$$
\left(W^{-(2), a}, G^{+\left(\frac{5}{2}\right), a}, G^{-\left(\frac{5}{2}\right), a}, W^{+(3), a}\right) \text {. }
$$

As described in the beginning of this section, it is an open problem to consider the nonsinglet spin- 2 current $K^{a}$ first and obtain the nonsinglet spin-3 current after acting the spin- $\frac{3}{2}$ currents of the $\mathcal{N}=2$ superconformal algebra on $K^{a}$ successively. Once we have obtained the nonsinglet spin-3 current, then it is straightforward to determine its superpartners step by step. We can check whether this procedure reproduces the above $\mathcal{N}=2$ multiplet in (5.12) or not.

In next subsections, further checks on the currents under the action of the currents of $\mathcal{N}=2$ superconformal algebra are given.
As before, the easy way to determine the various structure constants appearing in the right hand sides of the above OPEs is to figure out how the various composite operators arise from which parts of each current. For the last OPE of (5.13), it is rather nontrivial to keep track of the contributions from $t_{\rho \bar{\sigma}}^{\alpha} t_{j \bar{i}}^{a} t_{\rho_{1} \bar{v}}^{\alpha} \delta_{m \bar{m}} \psi^{(\bar{\sigma} j)}\left(\left(\psi^{\left(\rho_{1} \bar{m}\right)} J^{(\bar{v} m)}\right) J^{(\rho \bar{i})}\right)$ appearing in the sixth pair of brackets of the nonsinglet spin-3 current in (5.11) because of nontrivial normal ordering of the currents. Moreover, the presence of $W^{+2(a)}$ (which belongs to other $\mathcal{N}=2$ multiplet) in the second order pole can be understood from the fact that the above spin-3 current contains this current in various places of (5.11). In doing this, we also need the OPEs between $J_{f}^{a}(z)$ and $G^{ \pm, b}(w)$ which will be described in detail later.

Let us describe other OPEs as follows: 


$$
\begin{aligned}
& G^{+}(z) W^{-(2), a}(w)=\frac{1}{(z-w)} G^{+\left(\frac{5}{2}\right), a}(w)+\cdots, G^{+}(z) G^{+\left(\frac{5}{2}\right), a}(w)=0+\cdots, G^{+}(z) G^{-\left(\frac{5}{2}\right), a}(w) \\
& =\frac{1}{(z-w)^{3}} \frac{\left(k^{2}-1\right)\left(k^{2}-4\right)(k+M+N)(2 k+M+N)(3 k+2 M+2 N)}{k^{2}(k+M)(3 k+2 M)}\left[N J^{a}-k J_{f}^{a}\right](w) \\
& \quad+\frac{1}{(z-w)^{2}}(2 k+M+2 N) W^{-(2), a}(w)+\frac{1}{(z-w)}\left[\frac{1}{4} \partial(\text { pole- } 2)+i f^{a b c} J^{b} W^{-(2), c}+i f^{a b c} J_{f}^{b} W^{-(2), c}\right. \\
& \left.\quad+\frac{1}{4}(2 k+3 M+2 N) \partial W^{-(2), a}-W^{+(3), a}\right](w)+\cdots, \\
& G^{+}(z) W^{+(3), a}(w)=-\frac{1}{(z-w)^{3}} \frac{\left(k^{2}-1\right)\left(k^{2}-4\right)(k+N)(k+M+N)(2 k+M+N)(3 k+2 M+2 N)}{k^{2}(k+M)(3 k+2 M)} a_{1} \times G^{+, a}(w) \\
& \quad+\frac{1}{(z-w)^{2}} \frac{1}{4}(10 k+7 M+10 N) G^{+\left(\frac{5}{2}\right), a}+\frac{1}{(z-w)} \\
& {\left[\frac{1}{5} \partial(\text { pole- } 2)+i f^{a b c} J^{b} G^{+\left(\frac{5}{2}\right), c}+i f^{a b c} J_{f}^{b} G^{+\left(\frac{5}{2}\right), c}+\frac{2 M}{5} \partial G^{+\left(\frac{5}{2}\right), a}\right](w)+\cdots .}
\end{aligned}
$$

In the third OPE of (5.14), it is rather nontrivial to observe the first order pole explicitly. We should write down all the possible terms of spin- 3 with free index $a$. It turns out that the free index appears in the $f$ symbols and the currents themselves. This first order pole is another way to determine the nonsinglet spin-3 current. For the last OPE of (5.14), we can fix the coefficient of the last term appearing in the first order pole by using the property of quasi primary condition. That is, after calculating the third order pole in the OPEs between the stress energy tensor and the second and third terms of the first order pole and obtaining $-2 M G^{+\left(\frac{5}{2}\right), a}(w)$, we can fix the structure constant of $\partial G^{+\left(\frac{5}{2}\right), a}(w)$ as $\frac{2 M}{5}$ because we can check that the third order pole in the OPE between the stress energy tensor and this derivative term leads to $5 G^{+\left(\frac{5}{2}\right), a}(w)$. Then the last three terms appearing in the first order pole of the last OPE satisfy the quasi primary condition as we expected. $^{26}$

We continue to describe the next OPEs

$$
\begin{aligned}
& G^{-}(z) W^{-(2), a}(w)=\frac{1}{(z-w)} G^{-\left(\frac{5}{2}\right), a}(w)+\cdots, \\
& G^{-}(z) G^{+\left(\frac{5}{2}\right), a}(w)=-\frac{1}{(z-w)^{3}} \frac{\left(k^{2}-1\right)\left(k^{2}-4\right)(k+M+N)(2 k+M+N)(3 k+2 M+2 N)}{k^{2}(k+M)(3 k+2 M)} \times\left[N J^{a}-k J_{f}^{a}\right](w) \\
& \quad+\frac{1}{(z-w)^{2}}(2 k+M+2 N) W^{-(2), a}(w)+\frac{1}{(z-w)}\left[\frac{1}{4} \partial(\text { pole- } 2)+W^{+(3), a}\right](w)+\cdots, \\
& G^{-}(z) G^{-\left(\frac{5}{2}\right), a}(w)=0+\cdots, G^{-}(z) W^{+(3), a}(w)=\frac{1}{(z-w)^{3}} \\
& \quad \times \frac{\left(k^{2}-1\right)\left(k^{2}-4\right)(k+N)(k+M+N)(2 k+M+N)(3 k+2 M+2 N)}{k^{2}(k+M)(3 k+2 M)} a_{1} \\
& \quad \times G^{-, a}(w)-\frac{1}{(z-w)^{2}} \frac{5}{4}(2 k+M+2 N) G^{-\left(\frac{5}{2}\right), a}(w)-\frac{1}{(z-w)} \frac{1}{5} \partial(\text { pole- }-2)+\cdots .
\end{aligned}
$$

\footnotetext{
${ }^{26}$ In doing this, first we check the field contents for fixed $N$ and $M$ and then we need to figure out which parts of the currents of $\mathcal{N}=2$ multiplet contribute to the right hand sides of the OPEs. Next we calculate those contributions manually for generic $N$ and $M$.
} 
The second OPE of (5.15) provides the way we obtain the nonsinglet spin-3 current described before. In the last OPE of (5.15) which will be rather complicated compared to other OPEs above, we should also calculate all the contributions from the nonsinglet spin-3 current. Note that when we calculate the OPE between $G^{-}(z)$ and $i f^{a b c} G^{+, b} G^{-, c}(w)$ which is one of the terms of $W^{+(3), a}(w)$ and appears in the sixth line from the below, we should obtain the first order pole of the OPE between $W^{+(2), b}(z)$ and $G^{-, c}(w)$. See also the second OPE of (3.6). Then for example, the contribution of the fifth nonderivative term in $V^{-\left(\frac{5}{2}\right), a}(3.12)$ with (5.9) arises in this particular pole. The point here is, in general, the first order pole of the OPE between $W^{+(2), b}(z)$ and $G^{-, c}(w)$ produces a new quasi primary operator but with the contraction of indices $b$ and $c$ appearing in the $f$ symbol we can write down this first order pole in terms of the known currents. Therefore, there will be no new (quasi)primary field in the second order pole of the last OPE in (5.15). ${ }^{27}$ We will return to this issue around (6.5) in next section.

After collecting all the contributions on the fifth nontrivial term in $V^{-\left(\frac{5}{2}\right), a}(3.12)$ in this calculation of the OPE between $G^{-}(z)$ and $W^{+(3), a}(w)$, we are left with the structure constant in the second order pole as above. Moreover, the first order pole has the descendant terms only which can be checked by fixed $N$ and $M$.

Finally, the OPEs with the stress energy tensor are described by
Therefore, the nonsinglet currents are given by (5.7), (5.9) and (5.11) in the coset realization and they satisfy (5.13), (5.14), (5.15) and (5.16) under the action of the currents of $\mathcal{N}=2$ superconformal algebra. Also note that the right hand side of these OPEs contain the currents of $\mathcal{N}=2$ multiplet in (3.3) as well as the currents of $\mathcal{N}=2$ multiplet in (5.12).

\section{The OPEs between the nonsinglet multiplet of spins $\left(1, \frac{3}{2}, \frac{3}{2}, 2\right)$ and itself}

The simplest nontrivial OPEs between the nonsinglet $\mathcal{N}=2$ multiplets can be described in this section.

\subsection{The OPEs with lowest component}

From the explicit coset realizations in (2.3), (2.11) and (3.1), we determine the following OPEs (the OPEs between the lowest component and the four components in (3.3))

$$
\begin{aligned}
& J_{f}^{a}(z) J_{f}^{b}(w)=\frac{1}{(z-w)^{2}} N \delta^{a b} \\
& +\frac{1}{(z-w)} i f^{a b c} J_{f}^{c}(w)+\cdots, \\
& J_{f}^{a}(z) G^{+, b}(w)=\frac{1}{(z-w)}\left[-\frac{1}{M} \delta^{a b} G^{+}\right.
\end{aligned}
$$

$$
\begin{aligned}
& T(z) W^{-(2), a}(w)=\frac{1}{(z-w)^{2}} 2 W^{-(2), a}(w)+\frac{1}{(z-w)} \partial W^{-(2), a}(w)+\cdots, \\
& T(z) G^{ \pm\left(\frac{5}{2}\right), a}(w)=\frac{1}{(z-w)^{2}} \frac{5}{2} G^{ \pm\left(\frac{5}{2}\right), a}(w)+\frac{1}{(z-w)} \partial G^{ \pm\left(\frac{5}{2}\right), a}(w)+\cdots, \\
& T(z) W^{+(3), a}(w)=\frac{1}{(z-w)^{4}}\left[-\frac{3\left(k^{2}-1\right)\left(k^{2}-4\right)(k+M+N)(2 k+M+N)(3 k+2 M+2 N)}{2 k^{2}(k+M)(3 k+2 M)} a_{1}\left(N J^{a}-k J_{f}^{a}\right)\right](w) \\
& \quad+\frac{1}{(z-w)^{2}} 3 W^{+(3), a}(w)+\frac{1}{(z-w)} \partial W^{+(3), a}(w)+\cdots
\end{aligned}
$$

For the nonsinglet spin-3 current, there is a fourth order pole in (5.16). This implies that the nonsinglet spin-3 current is quasi primary field. By introducing the composite operator $K W^{-(2), a}$, we can make the following nonsinglet spin-3 current

$W^{+(3), a}+\frac{3}{2}(k+M+N) K W^{-(2), a}$

be primary. In doing this, the first OPE of (5.13) is used in $(5.17){ }^{28}$

27 This feature also appears in the previous last OPE of (5.14).
${ }^{28}$ In the OPE between $K(z)$ and $K W^{-(2), a}(w)$, the second order pole
contains $W^{-(2), a}$ and $K\left(N J^{a}-k J_{f}^{a}\right)$ terms. The OPE between $G^{+}(z)$
and $K W^{-(2), a}(w)$ leads to the second order pole with $G^{+\left(\frac{5}{2}\right), a}$ and

$$
\begin{aligned}
& \left.+\frac{1}{2}(i f+d)^{a b c} G^{+, c}\right](w)+\cdots, \\
& J_{f}^{a}(z) G^{-, b}(w)=\frac{1}{(z-w)}\left[\frac{1}{M} \delta^{a b} G^{-}\right. \\
& \left.+\frac{1}{2}(i f-d)^{a b c} G^{-, c}\right](w)+\cdots, \\
& J_{f}^{a}(z) W^{+(2), b}(w)=\frac{1}{(z-w)^{2}}\left[-\frac{1}{M}(k+M+N) \delta^{a b} K\right.
\end{aligned}
$$

Footnote 28 continued

the first order pole with $G^{+} W^{-(2), a}$ and $K G^{+\left(\frac{5}{2}\right), a}$. Furthermore, the OPE between $G^{-}(z)$ and $K W^{-(2), a}(w)$ becomes the second order pole with $G^{-\left(\frac{5}{2}\right), a}$ and the first order pole together with $G^{-} W^{-(2), a}$ and $K G^{-\left(\frac{5}{2}\right), a}$. There exist several nonlinear terms. 


$$
\begin{aligned}
- & \left.\frac{N}{2} i f^{a b c} J^{c}+\frac{1}{2} d^{a b c}\left(N J^{c}-k J_{f}^{c}\right)\right](w) \\
& +\frac{1}{(z-w)}\left[\frac{1}{2}(i f+d)^{c b d} i f^{a d e} J^{c} J_{f}^{e}\right. \\
& +i f^{a b c}\left(W^{+(2), c}-\frac{1}{k M}(k+M+N) J^{c} K\right. \\
& -\frac{1}{2}(i f+d)^{d c e} J^{d} J_{f}^{e}+\frac{N}{2} \partial J^{c} \\
& \left.\left.+\frac{1}{2}\left(K^{c}+\frac{N}{(2 k+M)} d^{c d e} J^{d} J^{e}-N \partial J^{c}\right)\right)\right](w) \\
& +\cdots
\end{aligned}
$$

In the last OPE of (6.1), we should replace the nonsinglet spin-2 current $K^{c}$ with the other spin-2 currents by using the nonsinglet spin-2 current (5.7) because the $K^{c}$ is not an element of $\mathcal{N}=2$ multiplet we are considering in the $\mathcal{N}=2$ supersymmetric coset model. It is obvious to see $J_{f}^{a}(z) K^{b}(w)=0+\cdots$ from Appendix A. The presence of the spin-2 current $K^{c}$ can be understood from the fact that in doing this, the unwanted terms, consisting of the second, third, fourth, and sixth terms of $W^{+(2), c}$ (3.1) with $f$ symbol after simplifying, are replaced by the nonsinglet spin-2 current $W^{+(2), c}$ and other terms which contain the nonsinglet spin- 2 current $K^{c}$. In the last line of (6.1), we replace the first term of $K^{c}$ (3.2) with other terms where $J^{c} J^{u(1)}$ term is combined with $J^{c} K$ term and to appear in the second line from the below.

From the last OPE of (6.1), we observe that the right hand side of the OPE contains also the lowest component of the $\mathcal{N}=2$ multiplet in (5.12).

\subsection{The OPEs with the second component}

Now let us consider the following OPEs (the OPEs between the second component and the three components in (3.3))

$$
\begin{aligned}
& G^{+, a}(z) G^{+, b}(w)=0+\cdots, \\
& G^{+, a}(z) G^{-, b}(w)=\frac{1}{(z-w)^{3}} k N \delta^{a b} \\
& \quad+\frac{1}{(z-w)^{2}}\left[\frac{(k+M+N)}{M} \delta^{a b} K+\frac{N}{2}(i f-d)^{a b c} J^{c}\right. \\
& \left.\quad+\frac{k}{2}(i f+d)^{a b c} J_{f}^{c}\right](w) \\
& \quad+\frac{1}{(z-w)}\left[\delta ^ { a b } \left(\frac{1}{M}(k+M+N) T-\frac{1}{2 M} J^{a} J^{a}\right.\right. \\
& \left.\quad-\frac{1}{2 M} J_{f}^{a} J_{f}^{a}+\frac{1}{2 M}(k+M+N) \partial K\right) \\
& \quad+i f^{a b c}\left(-\frac{1}{2} W^{+(2), c}-\frac{N}{4} \partial J^{c}+\frac{k}{4} \partial J_{f}^{c}-\frac{1}{2}\left(K^{c}\right.\right.
\end{aligned}
$$

$$
\begin{aligned}
+ & \left.\frac{N}{(2 k+M)} d^{c d e} J^{d} J^{e}-N \partial J^{c}\right) \\
+ & \frac{1}{4} d^{c d e} J^{d} J_{f}^{e}-\frac{1}{4} i f^{c d e} J^{d} J_{f}^{e} \\
+ & \left.\frac{(k+M+N)}{k M} J^{c} K\right) \\
+ & d^{a b c}\left(-\frac{1}{2} W^{+(2), c}-\frac{N}{4} \partial J^{c}+\frac{k}{4} \partial J_{f}^{c}\right. \\
+ & \left.\frac{1}{4} d^{c d e} J^{d} J_{f}^{e}-\frac{1}{4} i f^{c d e} J^{d} J_{f}^{e}\right) \\
- & \frac{1}{M} J^{b} J_{f}^{a}-\frac{1}{4}(i f+d)^{e b c} \\
\times & \left.(i f+d)^{a c d} J^{e} J_{f}^{d}\right](w)+\cdots \\
G^{+, a} & (z) W^{+(2), b}(w) \\
= & \frac{1}{(z-w)^{2}}\left[\frac{1}{2 M}(3 k+2 M+3 N) \delta^{a b} G^{+}\right. \\
& +\frac{1}{4}(N-k) i f^{a b c} G^{+, c} \\
& \left.-\frac{3}{4}(k+N) d^{a b c} G^{+, c}\right](w)+\frac{1}{(z-w)} \\
& \times\left[\frac{1}{3} \partial(\operatorname{pole}-2)+R^{+\left(\frac{5}{2}\right), a b}\right](w)+\cdots
\end{aligned}
$$

In Appendix D, we present the coset realization for the second OPE of (6.2).

The question is how we obtain the above result from Appendix D? For the second order pole, we can check them without any difficulty. For the first order pole, we can compute the $\delta^{a b}, i f^{a b c}$ and $d^{a b c}$ with the first order pole appearing in Appendix D. In other words, we have a free index $c$ for the last two cases while we do not have any free index for the first case. From the expression of $\left.\delta^{a b} G^{+, a}(z) G^{-, b}(w)\right|_{\frac{1}{(z-w)}}$, we can use the expression of stress energy tensor $T(2.5)$. Then it turns out that we have four independent terms appearing in the first two lines of the first order pole having $\delta^{a b}$ in (6.2).

From the expression of $\left.i f^{a b c} G^{+, a}(z) G^{-, b}(w)\right|_{\frac{1}{(z-w)}}$, we can use the expressions $W^{+(2), c}(3.1)$ and $K^{c}$ (3.2) in order to remove the unwanted terms. Then we have nine terms appearing in the second, third and fourth lines of the first order pole having $i f^{a b c}$ as well as $J^{c} J_{f}^{u(1)}$ term. From the expression of $\left.d^{a b c} G^{+, a}(z) G^{-, b}(w)\right|_{\frac{1}{(z-w)}}$, the previous relation (3.1) can be used. Then we have the five terms appearing in the fifth line of the first order pole having $d^{a b c}$ as well as $J^{c} J_{f}^{u(1)}$ term in (6.2).

Furthermore, the last term in Appendix D having the factor $t^{a} t^{c} t^{b}$ can be simplified and will appear in the last line 
of the first order pole as well as $J^{c} J_{f}^{u(1)}$ term. However, this $J^{c} J_{f}^{u(1)}$-term can be cancelled by the above two contributions. Therefore, we are left with the above final result where there is no $J^{c} J_{f}^{u(1)}$ term. ${ }^{29}$

In the last OPE of (6.2), there exist the following terms, after subtracting the descendant terms, which is a primary

$$
\begin{aligned}
& R^{+\left(\frac{5}{2}\right), a b}=\frac{(3 k+N)}{2 M} \delta^{a b} \partial G^{+}+\frac{1}{4}(-k-N) i f^{a b c} \partial G^{+, c} \\
& +\frac{1}{4}(-3 k-N) d^{a b c} \partial G^{+, c} \\
& -\sqrt{\frac{M+N}{M N}} i f^{a b c} J^{u(1)} G^{+, c} \\
& -\frac{(k M N+M+2 N)}{M N} \delta_{\rho \bar{\sigma}} \delta^{a b} \delta_{k \bar{i}} \psi^{(\bar{\sigma} k)} \partial J^{(\rho \bar{i})} \\
& -\frac{(k N+1)}{2 N} i f^{a b c} \delta_{\rho \bar{\sigma}} t_{k \bar{i}}^{c} \psi^{(\bar{\sigma} k)} \partial J^{(\rho \bar{i})} \\
& -\frac{(k M N+M+2 N)}{2 M N} d^{a b c} \delta_{\rho \bar{\sigma}} t_{k \bar{i}}^{c} \psi^{(\bar{\sigma} k)} \partial J^{(\rho \bar{i})} \\
& +i f^{a b c} t_{k \bar{i}}^{c} t_{\rho \bar{\mu}}^{\alpha} J^{(\rho \bar{i})} \psi^{(\bar{\mu} k)} J^{\alpha} \\
& +\frac{1}{M} J^{a} G^{+, b}-\frac{k}{M} \delta^{a b} \delta_{\tau \bar{\mu}} \delta_{j \bar{k}} J^{(\tau \bar{k})} \partial \psi^{(\bar{\mu} j)} \\
& +\frac{k}{2} i f^{a b c} \delta_{\tau \bar{\mu}} t_{j \bar{k}}^{c} J^{(\tau \bar{k})} \partial \psi^{(\bar{\mu} j)} \\
& -\frac{k}{2} d^{a b c} \delta_{\tau \bar{\mu}} t_{j \bar{k}}^{c} J^{(\tau \bar{k})} \partial \psi^{(\bar{\mu} j)} \\
& -\frac{M+N}{M N} t_{l \bar{k}}^{a} \delta_{\tau \bar{\mu}}\left(\left(\psi^{(\bar{\mu} l)} J^{(\tau \bar{k})}\right) J_{f}^{b}\right) \\
& -t_{\rho \bar{\sigma}}^{\alpha} t_{j \bar{i}}^{b} t_{l \bar{k}}^{a} t_{\mu \bar{\nu}}^{\alpha} \psi^{(\bar{\sigma} j)} \psi^{(\rho \bar{i})} \psi^{(\bar{v} l)} J^{(\mu \bar{k})} \\
& +\frac{1}{M^{2}} \delta^{a b} G^{+} J_{f}^{u(1)}-\frac{1}{2 M} i f^{a b c} G^{+, c} J_{f}^{u(1)} \\
& -\frac{1}{2 M} d^{a b c} G^{+, c} J_{f}^{u(1)}-\frac{1}{M} J^{b} G^{+, a} \\
& -\frac{1}{2 M}(i f+d)^{a b c} G^{+} J_{f}^{c} \\
& +\frac{1}{4}(i f+d)^{a c e}(i f+d)^{c b d} G^{+, e} J_{f}^{d} \\
& +\frac{1}{2 M}(i f+d)^{c b a} J^{c} G^{+} \\
& -\frac{1}{4}(i f+d)^{d a e}(i f+d)^{c b d} J^{c} G^{+, e} \\
& -\frac{1}{2}(i f+d)^{a c d}\left(t^{d} t^{b}\right)_{k \bar{i}} \delta_{\rho \bar{\sigma}} J^{(\bar{\rho} \bar{i})} \psi^{(\bar{\sigma} k)} J^{c} \\
& -\frac{1}{3} \partial\left(\left.G^{+, a}(z) W^{+(2), b}(w)\right|_{\frac{1}{(z-w)^{2}}}\right) .
\end{aligned}
$$

The question is whether this can be written in terms of the known currents or not. Let us look at $t_{\rho \bar{\sigma}}^{\alpha} t_{j \bar{i}}^{b} t_{l \bar{k}}^{a}$ $t_{\mu \bar{\nu}}^{\alpha} \psi^{(\bar{\sigma} j)} \psi^{(\rho \bar{i})} \psi^{(\bar{v} l)} J^{(\mu \bar{k})}$ term appearing in the sixth line

${ }^{29}$ For convenience, we present the OPEs $J^{a}(z) G^{ \pm, b}(w)=\frac{1}{(z-w)}[ \pm$ $\left.\frac{1}{M} \delta^{a b} G^{ \pm} \mp \frac{1}{2}(\mp i f+d)^{a b c} G^{ \pm, c}\right](w)+\cdots$. of (6.3). Although the index $\alpha$ is summed but the product of the generators of $S U(M)$ has the free indices $a$ and $b$ with four different lower indices contracted with the coset fields. As far as I know, there is no identity in the product of the two generators of $S U(M)$ with two different free indices. ${ }^{30}$

Therefore, we cannot express (6.3) in terms of the previous known currents obtained so far (although we have tried to rewrite it by using the various invariant tensors appearing in [12]). According to (1.3), each $\mathcal{N}=2$ current has a single $S U(M)$ index. It would be interesting to study how we can obtain the superpartners of (6.3) explicitly if they exist. It is natural to consider (6.3) as the second component of the $\mathcal{N}=$ 2 multiplet and then the lowest, the third and last components are not known so far.

\subsubsection{The OPE with spin-2 current}

Although the nonsinglet spin-2 current $K^{b}$ in the bosonic coset model does not belong to the component of the $\mathcal{N}=$ 2 multiplet, it is very useful to calculate the OPE between the nonsinglet spin- $\frac{3}{2}$ current $G^{+, a}$ of (3.3) and this spin-2 current. It turns out, from Appendix D where the coset field realizations are given, that we have

$$
\begin{aligned}
G^{+, a}(z) K^{b}(w)=\frac{1}{(z-w)^{2}} \frac{2\left(k^{2}-1\right)(2 k+M+N)}{k(2 k+M)} \\
\quad \times\left[\frac{1}{M} \delta^{a b} G^{+}-\frac{1}{2}(i f+d)^{a b c} G^{+, c}\right](w) \\
+\frac{1}{(z-w)}\left[-\frac{1}{3} \frac{2\left(k^{2}-1\right)(2 k+M+N)}{k(2 k+M)}\right. \\
\quad \times\left(\frac{1}{M} \delta^{a b} \partial G^{+}-\frac{1}{2}(i f+d)^{a b c} \partial G^{+, c}\right) \\
-\frac{1}{2}(i f+d)^{a b c}\left(V^{+\left(\frac{5}{2}\right), c}+\frac{2}{k M}(k+M+N) J^{c} G^{+}\right. \\
+\left(i f-\frac{(2 k+M+2 N)}{2 k+M} d\right)^{c d e} J^{d} G^{+, e} \\
\left.+\frac{2}{3} \frac{2\left(k^{2}-1\right)(2 k+M+N)}{k(2 k+M)} \partial G^{+, c}\right) \\
-\frac{2}{k M}(k+M+N) J^{b} G^{+, a} \\
+\frac{2 k(k+2 M)}{3\left(k^{2}-4\right)(k+M+N)(3 k+2 M+2 N) b_{1}} \\
\times \delta^{a b} G^{+\left(\frac{5}{2}\right), 0}
\end{aligned}
$$

30 There is a relation $t_{j \bar{i}}^{c} t_{l \bar{m}}^{d}=\delta^{c d} \delta_{j \bar{m}} \delta_{l \bar{i}}+\frac{M}{2}(i f+d)^{c d e} t_{j \bar{m}}^{e} \delta_{l \bar{i}}-$ $\frac{1}{M} \delta^{c d} \delta_{l \bar{m}} \delta_{j \bar{i}}-\frac{1}{2}(i f+d)^{c d e} t_{l \bar{m}}^{e} \delta_{j \bar{i}}-\frac{1}{2}(i f+d)^{c d e} t_{j \bar{i}}^{e} \delta_{l \bar{m}}-\frac{M}{4}(i f+$ $d)^{c a b}(i f+d)^{a d e} t_{j \bar{i}}^{b} t_{l \bar{m}}^{e}$. From this we obtain $i f^{a b c} t_{j \bar{i}}^{b} t_{l \bar{k}}^{a}=t_{j \bar{k}}^{c} \delta_{l \bar{i}}-$ $t_{l \bar{i}}^{c} \delta_{j \bar{k}}$ and $d^{a b c} t_{j \bar{i}}^{b} t_{l \bar{k}}^{a}=t_{j \bar{k}}^{c} \delta_{l \bar{i}}-\frac{2}{M} t_{j \bar{i}}^{c} \delta_{l \bar{k}}-\frac{2}{M} t_{l \bar{k}}^{c} \delta_{j \bar{i}}+t_{l \bar{i}}^{c} \delta_{j \bar{k}}$. In other words, when we contract with $f$ or $d$ symbols, then the product of two generators leads to several single generators with appropriate indices. We have seen these features in (5.14) and (5.15) also. 


$$
\begin{aligned}
& -\left(i f-\frac{(2 k+M+2 N)}{2 k+M} d\right)^{b c d} \\
& \times\left(\frac{1}{M} \delta^{a d} J^{c} G^{+}-\frac{1}{2}(i f+d)^{a d e} J^{c} G^{+, e}\right) \\
& -\frac{2}{3} \frac{2\left(k^{2}-1\right)(2 k+M+N)}{k(2 k+M)}\left(\frac{1}{M} \delta^{a b} \partial G^{+}\right. \\
& \left.\left.-\frac{1}{2}(i f+d)^{a b c} \partial G^{+, c}\right)\right](w)+\cdots .
\end{aligned}
$$

The first two lines of the first order pole in (6.4) are the descendant terms associated with the currents in the second order pole. Recall that the first, second and fifth terms in $V^{+\left(\frac{5}{2}\right), c}(3.10)$ appear as unwanted terms in the sense that they cannot be written in terms of the known currents. We can check that the second, fourth and last terms of the first order pole in the corresponding OPE in Appendix D can be written in terms of $V^{+\left(\frac{5}{2}\right), c}$ with $f$ and $d$ symbols plus other terms. Moreover, the spin- $\frac{5}{2}$ current $G^{+\left(\frac{5}{2}\right), 0}$ (4.5) contains similar first, third and fourth terms and they can be written in terms of $G^{+\left(\frac{5}{2}\right), 0}$ and other known operators. Finally, we arrives at the above results of the first order pole in (6.4) by matching those unwanted terms with $V^{+\left(\frac{5}{2}\right), c}, G^{+\left(\frac{5}{2}\right), 0}$ and other known composite operators. Of course, we can write down $V^{+\left(\frac{5}{2}\right), c}$ in terms of $G^{+\left(\frac{5}{2}\right), c}$ with other terms by using (5.9).

In summary, as before, from the second OPE in (6.2), we observe that the right hand side of the OPE contains the lowest component of the $\mathcal{N}=2$ multiplet in (5.12) by noting the presence of the nonsinglet spin-2 current. Moreover, from the last OPE, we observe that $i f^{a b c} t_{k \bar{i}}^{c} t_{\rho \bar{\mu}}^{\alpha} J^{(\rho \bar{i})} \psi^{(\bar{\mu} k)} J^{\alpha}$ term of (6.3) can be interpreted as $i f^{a b c} V^{+\left(\frac{5}{2}\right), c}$ (or $i f^{a b c} G^{+\left(\frac{5}{2}\right), c}$ ) plus other terms. This implies that the right hand side of the OPE contains the second component of the $\mathcal{N}=2$ multiplet in (5.12) as we expect.

\subsection{The OPEs with the third component}

The remaining two OPEs are given by

$$
\begin{aligned}
& G^{-, a}(z) G^{-, b}(w)=0+\cdots, \\
& G^{-, a}(z) W^{+(2), b}(w)=\frac{1}{(z-w)^{2}}\left[\frac{1}{2 M}(3 k+3 N) \delta^{a b} G^{-}\right. \\
& \quad-\frac{1}{4}(N-k) i f^{a b c} G^{-, c} \\
& \left.\quad-\frac{3}{4}(k+N) d^{a b c} G^{-, c}\right](w) \\
& +\frac{1}{(z-w)}\left[\frac{1}{3} \partial(\text { pole- } 2)+R^{-\left(\frac{5}{2}\right), a b}\right](w)+\cdots \cdot
\end{aligned}
$$

The first order pole in the last OPE of (6.5), after subtracting the descendant terms, contains the following primary spin- $\frac{5}{2}$ current with free indices $a$ and $b$

$$
\begin{aligned}
& R^{-\left(\frac{5}{2}\right), a b}=\frac{(k+N)}{2 M} \delta^{a b} \partial G^{-}-\sqrt{\frac{M+N}{M N}} i f^{a b c} J^{u(1)} G^{-, c} \\
& -\frac{(M+2 N)}{M^{2} N} \delta^{a b} \delta_{\rho \bar{\sigma}} \delta_{i \bar{k}} \psi^{(\sigma \bar{k})} \partial J^{(\bar{\rho} i)} \\
& +\frac{(2 k N+1)}{2 N} i f^{a b c} t_{k \bar{i}}^{c} \delta_{\rho \bar{\sigma}} \psi^{(\rho \bar{i})} \partial J^{(\bar{\sigma} k)} \\
& -\frac{(M+2 N)}{2 M N} d^{a b c} t_{k \bar{i}}^{c} \delta_{\rho \bar{\sigma}} \psi^{(\rho \bar{i})} \partial J^{(\bar{\sigma} k)} \\
& +i f^{a b c} t_{i \bar{k}}^{c} t_{\mu \bar{\rho}}^{\alpha} J^{(\bar{\rho} i)} \psi^{(\mu \bar{k})} J^{\alpha}-\frac{1}{M} J^{a} G^{-, b} \\
& +\frac{(M+N)}{M N} t_{k \bar{l}}^{a} \delta_{\mu \bar{\tau}}\left(\left(\psi^{(\mu \bar{l})} J^{(\bar{\tau} k)}\right) J_{f}^{b}\right) \\
& -t_{\sigma \bar{\rho}}^{\alpha} t_{i \bar{j}}^{b} t_{k \bar{l}}^{a} t_{v \bar{\mu}}^{\alpha} \psi^{(\sigma \bar{j})} \psi^{(\bar{\rho} i)} \psi^{(v \bar{l})} J^{(\bar{\mu} k)} \\
& -\frac{1}{M^{2}} \delta^{a b} G^{-} J_{f}^{u(1)}-\frac{1}{2 M} i f^{a b c} G^{-, c} J_{f}^{u(1)} \\
& +\frac{1}{2 M} d^{a b c} G^{-, c} J_{f}^{u(1)}+\frac{1}{M} J^{b} G^{-, a} \\
& +\frac{1}{2 M}(i f+d)^{a b d} G^{-} J_{f}^{d} \\
& -\frac{1}{4}(i f+d)^{c a e}(i f+d)^{c b d} G^{-, e} J_{f}^{d} \\
& -\frac{1}{2 M}(i f+d)^{c b a} J^{c} G^{-} \\
& -\frac{1}{4}(i f-d)^{d a e}(i f+d)^{c b d} J^{c} G^{-, e} \\
& +\frac{1}{4}(3 k+N) i f^{a b c} \partial G^{-, c} \\
& +\frac{1}{4}(-k-N) d^{a b c} \partial G^{-, c}+\frac{1}{2}(i f+d)^{c a d} t_{j \bar{i}}^{b} \delta_{\rho \bar{\sigma}} \\
& \times\left(t^{b} t^{d}\right) j \bar{l} J^{(\bar{\sigma} j)} \psi^{(\rho \bar{l})} J^{c} \\
& -\frac{1}{3} \partial\left(\left.G^{-, a}(z) W^{+(2), b}(w)\right|_{\frac{1}{(z-w)^{2}}}\right) \text {. }
\end{aligned}
$$

Again, due to the existence of $t_{\sigma \bar{\rho}}^{\alpha} t_{i \bar{j}}^{b} t_{k \bar{l}}^{a} t_{v \bar{\mu}}^{\alpha} \psi^{(\sigma \bar{j})} \psi^{(\bar{\rho} i)} \psi^{(v \bar{l})}$ $J^{(\bar{\mu} k)}$ in (6.6), we cannot express this in terms of the known currents. It is an open problem whether the above spin- $\frac{5}{2}$ current (6.6) is a third component of any $\mathcal{N}=2$ multiplet with free two indices. It is natural to consider the OPE between the spin- $\frac{3}{2}$ current $G^{-}$of the $\mathcal{N}=2$ superconformal algebra and the previous spin- $\frac{5}{2}$ current with two indices $R^{+\left(\frac{5}{2}\right), a b}(6.3)$ and obtain the possible lowest component of $\mathcal{N}=2$ multiplet. After that we need to check whether the OPE between the spin- $\frac{3}{2}$ current $G^{-}$and this lowest component (which will contain the quartic complex fermions with generators of $S U(N)$ and $S U(M)$ ) will give us the above spin- $\frac{5}{2}$ current (6.6) plus other terms or not. See also the footnote 31 for the four product of complex fermions. 


\subsubsection{The OPE with the spin-2 current}

As before, the corresponding OPE can be described as

$$
\begin{aligned}
& G^{-, a}(z) K^{b}(w)=-\frac{1}{(z-w)^{2}} \frac{2\left(k^{2}-1\right)(2 k+M+N)}{k(2 k+M)} \\
& \times\left[\frac{1}{M} \delta^{b a} G^{-}-\frac{1}{2}(i f+d)^{b a c} G^{-, c}\right](w) \\
& +\frac{1}{(z-w)}\left[-\frac{1}{3} \frac{2\left(k^{2}-1\right)(2 k+M+N)}{k(2 k+M)}\right. \\
& \times\left(\frac{1}{M} \delta^{b a} \partial G^{-}-\frac{1}{2}(i f+d)^{b a c} \partial G^{-, c}\right) \\
& -\frac{1}{2}(i f+d)^{b a c}\left(V^{-\left(\frac{5}{2}\right), c}-\frac{2}{k M}(k+M+N) J^{c} G^{-}\right. \\
& +\left(i f+\frac{(2 k+M+2 N)}{2 k+M} d\right)^{c d e} J^{d} G^{-, e} \\
& \left.+\frac{2}{3} \frac{2\left(k^{2}-1\right)(2 k+M+N)}{k(2 k+M)} \partial G^{-, c}\right) \\
& +\frac{2}{k M}(k+M+N) J^{b} G^{-, a} \\
& -\frac{2 k(k+2 M)}{3\left(k^{2}-4\right)(k+M+N)(3 k+2 M+2 N) b_{1}} \\
& \times \delta^{a b} G^{-\left(\frac{5}{2}\right), 0} \\
& -\left(i f+\frac{(2 k+M+2 N)}{2 k+M} d\right)^{b c d} \\
& \times\left(\frac{1}{M} \delta^{d a} J^{c} G^{-}-\frac{1}{2}(i f+d)^{d a e} J^{c} G^{-, e}\right) \\
& -\frac{2}{3} \frac{2\left(k^{2}-1\right)(2 k+M+N)}{k(2 k+M)} \\
& \left.\times\left(\frac{1}{M} \delta^{b a} \partial G^{-}-\frac{1}{2}(i f+d)^{b a c} \partial G^{-, c}\right)\right](w) \\
& +\cdots \text {. }
\end{aligned}
$$

The second, fourth and last terms of the first order pole in the corresponding OPE in Appendix D can be written in terms of $V^{-\left(\frac{5}{2}\right), c}$ with $f$ and $d$ symbols plus other terms. By using the first, second and fifth terms in $V^{-\left(\frac{5}{2}\right), c}$ (3.12) appearing as unwanted terms, we can reexpress the corresponding terms in the first order pole in terms of $V^{-\left(\frac{5}{2}\right), c}$ and other known operators. From the first, third and fourth terms in the spin$\frac{5}{2}$ current $G^{-\left(\frac{5}{2}\right), 0}(4.6)$, the corresponding terms in the first order pole can be written in terms of $G^{-\left(\frac{5}{2}\right), 0}$ and other known operators by focusing on the $\delta^{a b}$ factor in the product of two $S U(M)$ generators with indices $a$ and $b$. Finally, we obtain the above results (6.7) where we can write down $V^{-\left(\frac{5}{2}\right), c}$ in terms of $G^{-\left(\frac{5}{2}\right), c}$ with other terms by using (5.9).

\subsection{The final OPE}

Now we describe the final OPE between the spin-2 current and itself with Appendix E

$$
\begin{aligned}
& W^{+(2), a}(z) W^{+(2), b}(w)=\frac{1}{(z-w)^{4}} \frac{3}{2} k N(k+N) \delta^{a b} \\
& +\frac{1}{(z-w)^{3}}\left[\frac{1}{2} N(2 k+N) i f^{a b c} J^{c}+\frac{1}{2} k(k+2 N) i f^{a b c} J_{f}^{c}\right](w) \\
& +\frac{1}{(z-w)^{2}}\left[\delta ^ { a b } 2 ( k + N ) \left(\frac{1}{M}(k+M+N) T\right.\right. \\
& \left.-\frac{1}{2 M} J^{c} J^{c}-\frac{1}{2 M} J_{f}^{c} J_{f}^{c}\right) \\
& +d^{a b c}\left(-(k+N) W^{+(2), c}+\frac{1}{2}(i f+d)^{d c e} J^{d} J_{f}^{e}\right. \\
& \left.+\frac{k}{2}(1-k-N) \partial J_{f}^{c}+\frac{1}{2} N(k+N-1) \partial J^{c}\right) \\
& +i f^{a b c}\left(\frac{k}{4}(k+2 N) \partial J_{f}^{c}\right. \\
& \left.+\frac{N\left(2 k^{3} M+k^{2} M^{2}+k^{2} M N+2 k M+4 k N+M^{2}+M N\right)}{2 k M(2 k+M)} \partial J^{c}\right) \\
& -\frac{(k+2 M+N)}{M} J^{b} J_{f}^{a}-\frac{(k+N)}{M} J^{a} J_{f}^{b} \\
& -\frac{k}{4}(i f+d)^{d a e}(i f+d)^{c b d} J^{c} J_{f}^{e} \\
& -\frac{k}{4}(i f+d)^{d b e}(i f+d)^{c a d} J^{c} J_{f}^{e} \\
& -\frac{1}{4} f^{d h f} f^{e c g}(i f+d)^{h a c}(i f+d)^{d b e} J^{f} J_{f}^{g} \\
& -\frac{k}{4} i f^{b c g}(i f+d)^{d a c} J^{d} J_{f}^{g}-\frac{N}{4} d^{e b c}(i f+d)^{e a d} J^{c} J_{f}^{d} \\
& -\frac{k}{4} i f^{a c g}(i f+d)^{d b c} J^{d} J_{f}^{g} \\
& \left.-\frac{N}{4} d^{e a c}(i f+d)^{e b d} J^{c} J_{f}^{d}\right](w) \\
& +\frac{1}{(z-w)}\left[\frac{1}{2} \partial(\text { pole- } 2)+R^{+(3), a b}\right](w)+\cdots .
\end{aligned}
$$

In the second order pole of (6.8), we observe that there exists $d^{a b c} W^{+(2), c}$ term. Moreover, there are no quartic terms in the complex fermions by collecting all the contributions. ${ }^{31}$ In the first order pole of (6.8), after subtracting the descendant terms, we are left with $R^{+(3), a b}$ terms. In particular, we observe that by checking the purely bosonic terms in $R^{+(3), a b}$, there is a term

There is a relation $k \frac{M+N}{M N} J_{f}^{a} J_{f}^{b}+$ $k t_{\rho \bar{\sigma}}^{\alpha} t_{\mu \bar{\nu}}^{\alpha} t_{j \bar{i}}^{a} t_{k \bar{l}}^{b}\left(\psi^{(\rho \bar{i})} \psi^{(\bar{\sigma} j)}\right)\left(\psi^{(\mu \bar{l})} \psi^{(\bar{\nu} k)}\right)+\frac{k}{M^{2}} \delta^{a b} J_{f}^{u(1)} J_{f}^{u(1)}-$ $\frac{k}{M} d^{a b c} J_{f}^{c} J_{f}^{u(1)}+\frac{k}{4}(i f+d)^{d a c}(i f+d)^{d b e} J_{f}^{c} J_{f}^{e}=0$. That is, the exact coefficients appearing in these five terms with the appropriate complex fermion terms lead to zero. Note that the particular combination of this quartic fermions (with two indices $a$ and $b$ ) $t_{\rho \bar{\sigma}}^{\alpha} t_{\mu \bar{\nu}}^{\alpha} t_{j \bar{i}}^{a} t_{k \bar{l}}^{b} \psi^{(\rho \bar{i})} \psi^{(\bar{\sigma} j)} \psi^{(\mu \bar{l})} \psi^{(\bar{v} k)}$ is a candidate term of the lowest component of $\mathcal{N}=2$ multiplet with two free indices. 


$$
\begin{aligned}
& \frac{2 k(k+M)(3 k+2 M)}{\left(k^{2}-4\right)(2 k+M)(3 k+2 M+2 N)(3 k+M+3 N)} \\
& \frac{1}{a_{1}} i f^{a b c} W^{+(3), c} .
\end{aligned}
$$

This can be seen from the OPE between $K^{a}$ and $K^{b}$ and the $a_{1}$ term of $P^{c}$ appears in the inside of the sixth pair of bracket in (5.11). The nonsinglet spin-3 current $W^{+(3), c}$ in (6.9) is the last component of the $\mathcal{N}=2$ multiplet in (5.12). This is reasonable because the OPE between $K^{a}(z)$ and $K^{b}(w)$ leads to $i f^{a b c} P^{c}$ term at the first order pole in the bosonic coset model [12].

One of the reasons why we cannot write down this $R^{+(3), a b}$ in terms of the known currents is that there is a term from the OPE between the fourth term of $W^{+(2), a}$ (3.1)and itself (which is presented in Appendix E)

$i f^{\alpha \beta \gamma} t_{\rho \bar{\sigma}}^{\alpha} t_{\sigma \bar{v}}^{\beta} t_{j \bar{i}}^{b} t_{l \bar{k}}^{a} J^{\gamma} \psi^{(\rho \bar{i})} \psi^{(\bar{\sigma} j)} \psi^{(\sigma \bar{k})} \psi^{(\bar{v} l)}$,
In summary, we have the complete OPEs in (6.1), (6.2), (6.5) and (6.8). The nontrivial part of these OPEs is that in the right hand side of these OPEs, the components of the third $\mathcal{N}=2$ multiplet (5.12) as well as the components of the first $\mathcal{N}=2$ multiplet (3.3) and the currents of $\mathcal{N}=2$ superconformal algebra arise. We have seen the new primary currents having two free indices of $S U(M)$. The various quasi primary operators in the first order pole of (6.8) will appear as in the bosonic case.

\section{Towards the OPEs between the singlet multiplet of spins $\left(2, \frac{5}{2}, \frac{5}{2}, 3\right)$ and itself}

The simplest OPE between the singlet currents is given by the OPE between the singlet spin-2 current and itself. We obtain the following OPE with the help of Appendix F

$$
\begin{aligned}
& W^{-(2), 0}(z) W^{-(2), 0}(w)=\frac{1}{(z-w)^{4}}\left[\frac{6\left(k^{2}-1\right)\left(k^{2}-4\right)^{2} N(k+M+N)^{3}(2 k+M+N)(3 k+2 M+2 N)^{2}}{k^{3} M(k+M)^{2}(k+2 M)^{2}}\right. \\
& \left.\times\left(k^{3}+2 k^{2} M+2 k^{2} N+3 k M N+2 k+M+N\right) b_{1}^{2}\right] \\
& +\frac{1}{(z-w)^{2}}\left[\frac{8\left(k^{2}-1\right)\left(k^{2}-4\right)^{2}(k+M+N)^{4}(2 k+M+N)(3 k+2 M+2 N)^{2}}{k^{4} M^{2}(k+M)^{2}(k+2 M)^{2}}\right. \\
& \times\left(k^{3}+2 k^{2} M+2 k^{2} N+3 k M N+2 k+M+N\right) b_{1}^{2} \\
& \left(T-\frac{1}{2(k+M+N)}\left(J^{a}+J_{f}^{a}\right)^{2}\right)+\frac{4\left(k^{2}-4\right)(k+M+N)^{2}(3 k+2 M+2 N)}{k^{2} M(k+M)(k+2 M)} \\
& \left.\times\left(k^{3}-k^{2} M-k^{2} N-3 k M N-4 k-2 M-2 N\right) b_{1} W^{-(2), 0}\right](w)+\frac{1}{(z-w)} \frac{1}{2} \partial(\text { pole- } 2)(w)+\cdots .
\end{aligned}
$$

which can be obtained by acting $G^{-}(z)$ on $t_{\rho \bar{\sigma}}^{\alpha} t_{j \bar{i}}^{b} t_{l \bar{k}}^{a} t_{\mu \bar{v}}^{\alpha}$ $\psi^{(\bar{\sigma} j)} \psi^{(\rho \bar{i})} \psi^{(\bar{v} l)} J^{(\mu \bar{k})}(w)$. This spin- $\frac{5}{2}$ operator is the characteristic term for the $R^{+\left(\frac{5}{2}\right), a b}$ in (6.3). Then the OPE between $G^{-}$and $R^{+\left(\frac{5}{2}\right), a b}$ contains the above term (6.10). Note from the footnote 22 that the OPE between $G^{-}$and $J^{\gamma}$ leads to the composite field of spin- $\frac{1}{2}$ and spin-1 operators transforming as $(\mathbf{N}, \overline{\mathbf{M}})$ and $(\overline{\mathbf{N}}, \mathbf{M})$ respectively. Therefore we obtain (6.10) after contracting the indices properly. In this way, there is a connection between $R^{+\left(\frac{5}{2}\right), a b}$ and $R^{+(3), a b}$ via a supersymmetry generator. It is an open problem to see whether there exists any $\mathcal{N}=2$ multiplet of $\left(R^{-(2), a b}, R^{+\left(\frac{5}{2}\right), a b}, R^{-\left(\frac{5}{2}\right), a b}, R^{+(3), a b}\right)$ or not. Probably, if we compute the OPEs between the currents of high spins, then the lowest current $R^{-(2), a b}$ can be seen. ${ }^{32}$

\footnotetext{
32 Because we present all the OPEs between the nonsinglet spin-2 operators in Appendix E, by reversing the orders of some OPEs we can write all the contributions on the first order poles in (6.8) and read off
}

From the fourth order pole of (7.1), we can fix the normalization of the singlet spin- 2 current by taking the unknown coefficient $b_{1}^{2}$ properly. For example, one way to fix is such that the fourth order pole is given by $\frac{c}{2}$ where $c$ is the central charge in (2.6). The self coupling constant of the singlet spin-2 current which depends on the $k, N$ and $M$ explicitly appears in the second order pole of (7.1). As we expected, the right sides of this OPE consist of (1) the central term, (2) stress energy tensor, (3) $\left(J^{a}+J_{f}^{a}\right)^{2}$ term, (4) the singlet spin-2 current and (5) their descendant terms. ${ }^{33}$

Footnote 32 continued

the nonsinglet spin-3 current $R^{+(3), a b}$ explicitly which covers a several pages.

${ }^{33}$ It is useful to use the following relation $\delta_{\rho \bar{\sigma}} \delta_{j \bar{i}} J^{(\rho \bar{i})} J^{(\bar{\sigma} j)}=$ $(k+M+N) T_{\text {boson }}+\frac{M}{2(k+N)} J^{\alpha} J^{\alpha}+\frac{(M+N)}{2 k} J^{u(1)} J^{u(1)}-\frac{1}{2} J^{a} J^{a}+$ $\frac{1}{2} M N \sqrt{\frac{M+N}{M N}} \partial J^{u(1)}$ together with the footnote 9 in order to calculate the OPE between the first term of $W^{-(2), 0}$ and itself. Then we know the OPE between $T_{\text {boson }}$ and itself and the four remaining composite 
We can check that the singlet spin-2, $\frac{5}{2}, 3$ currents, $W^{-(2), 0}, G^{ \pm\left(\frac{5}{2}\right), 0}$ and $W^{+(3), 0}$ have the regular terms in the OPEs with $\left(J^{a}+J_{f}^{a}\right)$. Moreover, the OPE between the combination of $T-\frac{1}{2(k+M+N)}\left(J^{a}+J_{f}^{a}\right)^{2}$ and $\left(J^{a}+J_{f}^{a}\right)$ does not have any singular terms. This implies that the singlet currents,

$$
\begin{aligned}
& T-\frac{1}{2(k+M+N)}\left(J^{a}+J_{f}^{a}\right)^{2}, \quad W^{-(2), 0}, \\
& G^{ \pm\left(\frac{5}{2}\right), 0}, \quad W^{+(3), 0},
\end{aligned}
$$

are decoupled from the spin-1 current $\left(J^{a}+J_{f}^{a}\right)$. It is easy to observe that the previous (quasi)primary conditions for these singlet spin- $2, \frac{5}{2}, 3$ currents still hold under the modified stress energy tensor because the $\left(J^{a}+J_{f}^{a}\right)^{2}$ term does not produce any singular terms. ${ }^{34}$

After the OPEs between the currents of $\left(W^{-(s), 0}\right.$, $\left.G^{+\left(s+\frac{1}{2}\right), 0}, G^{-\left(s+\frac{1}{2}\right), 0}, W^{+(s+1), 0}\right) \quad$ in (1.3) where $s=2,3,4, \cdots$ are obtained, we expect that the right hand sides of these OPEs will consist of the composite operators in terms of these singlet currents as well as the modified stress energy tensor (7.2) (and maybe $K$ and $G^{ \pm}$also). The new singlet (quasi)primary current will have the regular OPE with the spin-1 current $\left(J^{a}+J_{f}^{a}\right)$.

Then the algebra from $\left(W^{-(s), 0}, G^{+\left(s+\frac{1}{2}\right), 0}, G^{-\left(s+\frac{1}{2}\right), 0}\right.$, $W^{+(s+1), 0}$ ) will close by themselves up to the presence of the currents of $\mathcal{N}=2$ superconformal algebra with modified stress energy tensor. In other words, the right hand sides of these OPEs do not contain the $S U(M)$ nonsinglet fields appearing in (1.3). On the other hands, the OPEs between the nonsinglet currents do contain the $S U(M)$ singlet fields.

\section{The extension of the large $\mathcal{N}=4$ nonlinear superconformal algebra for $M=2$}

We describe in this section how we can realize the extension of the large $\mathcal{N}=4$ nonlinear superconformal algebra for $M=2$.

\subsection{Four spin- $\frac{3}{2}$ currents}

The four supersymmetry generators of the large $\mathcal{N}=4$ nonlinear superconformal algebra can be obtained by combining $G^{+, a}$ with $G^{-, a}$ and also combining $G^{+}$and $G^{-}$properly

Footnote 33 continued

spin-2 operators of above belong to $W^{-(2), 0}$ and we can also compute the OPEs between them.

34 We can see that the OPEs between $\left(J^{a}+J_{f}^{a}\right)$ and $K, G^{ \pm}$do not produce any singular terms.

$$
\begin{aligned}
& \hat{G}_{11}=\sqrt{\frac{1}{k+N+2}}\left[G^{+, 1}-i G^{+, 2}+G^{-, 1}-i G^{-, 2}\right], \\
& \hat{G}_{12}=-\frac{1}{\sqrt{2}} \sqrt{\frac{1}{k+N+2}}\left[G^{+}+\sqrt{2} G^{+, 3}-G^{-}+\sqrt{2} G^{-, 3}\right] \text {, } \\
& \hat{G}_{21}=\frac{1}{\sqrt{2}} \sqrt{\frac{1}{k+N+2}}\left[G^{+}-\sqrt{2} G^{+, 3}-G^{-}-\sqrt{2} G^{-, 3}\right] \text {, } \\
& \hat{G}_{22}=\sqrt{\frac{1}{k+N+2}}\left[G^{+, 1}+i G^{+, 2}+G^{-, 1}+i G^{-, 2}\right] .
\end{aligned}
$$

These expressions (8.1) satisfy the fundamental relations Appendix (D.1) of [72] or Appendix (G.1) in this paper. ${ }^{35}$ From the explicit expressions in $G^{ \pm}(2.7)$ and $G^{ \pm, a}(2.11)$, the summation over the fundamental and antifundamental $S U(N)$ indices appears in the Kronecker delta with the coset fields. On the other hands, the summation over the fundamental and antifundamental $S U(M=2)$ indices appears in the matrix elements of the $S U(M=2)$ generators with the coset fields. For example, in the spin- $\frac{3}{2}$ current $\hat{G}_{11}$, the index $j=1$ and the index $\bar{i}=2$ survives in the first combination of $\left(G^{+, 1}-i G^{+, 2}\right)$ and the index $j=2$ and the index $\bar{i}=1$ survives in the second combination of $\left(G^{-, 1}-i G^{-, 2}\right)$. This corresponds to the last $4 N \times 4 N$ matrix in Appendix (B.2) of [72] where the nonzero elements appear in the two $N \times N$ identity matrices inside of this matrix. We can analyze the other three currents similarly from $G^{ \pm}(2.7)$ and $G^{ \pm, a}(2.11)$.

\subsection{Higher spin- $\frac{3}{2}$ currents}

Then the next spin- $\frac{3}{2}$ currents can be determined by using the relations Appendix (G.1) of [72] (or Appendix (G.2) in this paper) and it turns out that

$$
\begin{aligned}
T_{+}^{\left(\frac{3}{2}\right)} & =-\frac{1}{\sqrt{2}} \sqrt{\frac{1}{k+N+2}}\left[G^{+}-\sqrt{2} G^{+, 3}\right], \\
T_{-}^{\left(\frac{3}{2}\right)} & =\frac{1}{\sqrt{2}} \sqrt{\frac{1}{k+N+2}}\left[G^{-}-\sqrt{2} G^{-, 3}\right], \\
U^{\left(\frac{3}{2}\right)} & =-\sqrt{\frac{1}{k+N+2}}\left[G^{+, 1}-i G^{+, 2}\right], \\
V^{\left(\frac{3}{2}\right)} & =\sqrt{\frac{1}{k+N+2}}\left[G^{-, 1}+i G^{-, 2}\right] .
\end{aligned}
$$

Again, from (2.7) and (2.11), we observe that in the spin- $\frac{3}{2}$ current $T_{+}^{\left(\frac{3}{2}\right)}$, the index $j=1$ and the index $\bar{i}=1$ sur-

35 We have the following six spin-1 currents $\hat{A}_{ \pm}=\frac{1}{\sqrt{2}}\left(-i J^{1} \mp J^{2}\right)$, $\hat{A}_{3}=-\frac{i}{\sqrt{2}} J^{3}$ and $\hat{B}_{ \pm}=\frac{1}{\sqrt{2}}\left(i J_{f}^{1} \mp J_{f}^{2}\right), \hat{B}_{3}=\frac{i}{\sqrt{2}} J_{f}^{3}$. The defining relations for these $S U$ (2) currents are given in (2.13) and (2.16) of [72] where we can fix the normalizations of the spin- 1 currents. The defining OPEs between these spin- 1 current and four spin- $\frac{3}{2}$ currents are given in Appendix C of [72]. Furthermore, the spin-1, $\frac{3}{2}$ currents are primary under the stress energy tensor. The central charge (2.6) gives us $c=\frac{3(k+N+2 k N)}{(k+2+N)}$ for $M=2$. 
vives and this corresponds to the nonzero elements in the $N \times N$ identity matrix inside of the $4 N \times 4 N$ matrix in the last expression of Appendix B of [72]. Here, each of spin$\frac{3}{2}$ currents in (8.1) is reduced to further here. Note that the eight rank two tensors which are $4 N \times 4 N$ matrices and the nonzero elements appear in four $N \times N$ identity matrices in [72]. In (8.2), we are left with these $4 N \times 4 N$ matrices where only $N \times N$ identity matrix arises.

Then the eight spin- $\frac{3}{2}$ currents of the left hand sides in (8.1) and (8.2) for generic $k$ and $N$ can be realized by two $G^{ \pm}$and six $G^{ \pm, a}$ (where $a=1,2,3$ ) in the right hand sides.

\subsection{Higher spin-2 currents}

By using Appendix (G.2) of [72] together with (8.1) and (8.2) (or Appendix (G.3)), we can determine the following spin-2 currents for generic $k$ and $N$ as follows:

$$
\begin{aligned}
U_{-}^{(2)}= & -\frac{1}{2(k+N+2)}\left[-i f^{31 c} K^{c}\right. \\
& +\frac{(k+N+2)}{k} i f^{31 c} J^{c} K+2 J^{1} J_{f}^{3} \\
& \left.-f^{32 c} K^{c}+\frac{(k+N+2)}{k} f^{32 c} J^{c} K-2 i J^{2} J_{f}^{3}\right], \\
V_{+}^{(2)}= & \frac{1}{2(k+N+2)}\left[i f^{31 c} K^{c}\right. \\
& -\frac{(k+N+2)}{k} i f^{31 c} J^{c} K+2 J^{1} J_{f}^{3} \\
& \left.-f^{32 c} K^{c}+\frac{(k+N+2)}{k} f^{32 c} J^{c} K+2 i J^{2} J_{f}^{3}\right], \\
U_{+}^{(2)}= & -\frac{1}{2(k+N+2)}\left[2 i f^{13 c} W^{+(2), c}+i f^{13 c} K^{c}\right. \\
& -\frac{1}{k} i f^{13 c} J^{c} K+4 J^{3} J_{f}^{1} \\
& +f^{a 3 c} f^{1 c d} J^{a} J_{f}^{d}+2 f^{23 c} W^{+(2), c} \\
& +f^{23 c} K^{c}-\frac{(k+N+2)}{k} f^{23 c} J^{c} K \\
& \left.-4 i J^{3} J_{f}^{2}-i f^{a 3 c} f^{2 c d} J^{a} J_{f}^{d}\right], \\
T^{(2)}= & -\frac{1}{2(k+N+2)}\left[-\frac{2(k+N)(k+N+2)}{(k+N+2 k N)} \delta^{33} T\right. \\
& +2 J^{3} J_{f}^{3} \\
V_{-}^{(2)}= & \frac{(k+N+2)}{2(k+N+2)}\left[2 i f^{31 c} W^{+(2), c}+i f^{31 c} K^{c}\right. \\
& \left.-\frac{(k+N+2)}{k} i f^{31 c} J^{c} K+2 J^{1} J_{f}^{3} K+2 i J^{2} J_{f}^{3}\right], \\
& -2 f^{32 c} W^{+(2), c}-f^{32 c} K^{c} \\
& (k+N)
\end{aligned}
$$

$$
\begin{aligned}
& +\sqrt{2} i f^{3 a b} J^{a} J_{f}^{b} \\
& \left.+J^{a} J^{a}+J_{f}^{a} J_{f}^{a}+2 \sqrt{2} W^{+(2), 3}\right], \\
W^{(2)}= & \frac{1}{2(k+N+2)}\left[-2 J^{1} J_{f}^{1}\right. \\
& -2 f^{21 c} W^{+(2), c}-2 f^{21 c} K^{c} \\
& +\frac{2(k+N+2)}{k} f^{21 c} J^{c} K \\
& +2 i J^{1} J_{f}^{2}+i f^{a 1 c} f^{2 c d} J^{a} J_{f}^{d} \\
& +2(k+N+2) \delta^{22} T-\delta^{22} J^{a} J^{a} \\
& \left.-\delta^{22} J_{f}^{a} J_{f}^{a}+f^{a 2 c} f^{2 c d} J^{a} J_{f}^{d}\right] .
\end{aligned}
$$

The way we obtain $(8.3)$ is that for fixed $(N, M)=(5,2)$, we can determine the field contents explicitly. After that, we read off the generic $(N, M)$ dependence manually. If we compute the relevant OPEs manually from the beginning, then we will obtain different expressions. However, we can check that eventually those become the above results (8.3) by using some identities in the structure constants. In (8.3), although there exist the spin- 2 current $K^{a}$ dependent terms, we can replace them by using (5.7) with $W^{ \pm(2), a}$ term and others.

Therefore, the six spin-2 currents for generic $k$ and $N$ can be realized by the six spin- 2 currents $W^{ \pm(2), a}$ where $a=1,2,3$ and other composite operators. ${ }^{36}$

\subsection{Higher spin- $\frac{5}{2}, 3$ currents}

Now we can compute Appendix (G.4) of [72] by using (8.1) and (8.3) and taking the first order poles (or Appendix (G.4)), we will obtain the four spin- $\frac{5}{2}$ currents. Then we need to calculate the OPEs between $G^{ \pm, a}(z)$ and $W^{ \pm(2), b}(w)$ as done in Sect. 6. In other words, we should compute the OPEs between the second $\mathcal{N}=2$ multiplet and itself (and the OPEs between the first $\mathcal{N}=2$ multiplet and the second $\mathcal{N}=2$ multiplet). For fixed $N$ with $M=2$, we have checked that we can write down the spin- $\frac{5}{2}$ currents explicitly. See Appendix

\footnotetext{
36 By considering the second order poles of the last four OPEs in Appendix (G.2) of [72], the spin-1 current of the lowest $\mathcal{N}=4$ higher spin multiplet can be realized by $K$ in the $\mathcal{N}=2$ superconformal algebra in (2.9). Moreover, the stress energy tensor of the $\mathcal{N}=4$ large nonlinear superconformal algebra is identified with the stress energy tensor in (2.9). The stress energy tensor consists of purely bosonic part, purely fermionic part and boson fermionic part. The purely fermionic part can be summarized by the first two terms in the second line of $T(2.5)$ and $J_{f}^{a} J_{f}^{a}$ term after using the identity appearing in the last equation of Appendix A. The boson fermionic part is given by both $J^{\alpha} J_{f}^{\alpha}$ term and $J^{u(1)} J_{f}^{u(1)}$ term. The purely bosonic part consists of the second, third and fourth terms of $T$ (2.5). Then we can make the correspondences between the stress energy tensor in [72] and the one in (2.5) by focusing on these three parts.
} 
(H.1). It turns out that they can be written in terms of $V^{ \pm\left(\frac{5}{2}\right), a}$ (or $G^{ \pm\left(\frac{5}{2}\right), a}$ ) plus other terms. ${ }^{37}$

Eventually, the spin-3 current can be realized by the equation (3.50) of [72]. That is, after determining the spin- $\frac{5}{2}$ current $W_{-}^{\left(\frac{5}{2}\right)}$ for generic $N$ and $M$, we use

$$
\begin{aligned}
W^{(3)}= & \left.\hat{G}_{21}(z) W_{-}^{\left(\frac{5}{2}\right)}(w)\right|_{\frac{1}{(z-w)}} \\
& -\left[\frac{1}{4} \partial\left(\left.\hat{G}_{21}(z) W_{-}^{\left(\frac{5}{2}\right)}(w)\right|_{\frac{1}{(z-w)^{2}}}\right)\right. \\
& +\frac{8 i N(3 k+1)}{(N+k+2)(5 N+4+6 k N+5 k)}\left(-\frac{i}{\sqrt{2}}\right)\left(T J^{3}\right. \\
& \left.-\frac{1}{2} \partial^{2} J^{3}\right) \\
& +\frac{8 i k(3 N+1)}{(N+k+2)(5 N+4+6 k N+5 k)}\left(\frac{i}{\sqrt{2}}\right)\left(T J_{f}^{3}\right. \\
& \left.-\frac{1}{2} \partial^{2} J_{f}^{3}\right) \\
& \left.+\frac{8(k-N)}{(5 N+4+6 k N+5 k)}\left(T K-\frac{1}{2} \partial^{2} K\right)\right] .
\end{aligned}
$$

Of course, from the first line of (8.4), we should calculate the corresponding OPEs. In the last three lines, the relations appearing in the footnotes 35 and 36 are used.

\subsection{For $M=3$}

What happens for $M=3$ case? In particular, how do the supersymmetry generators appear when we increase $M$ by 1 ? The field contents for the spin- $\frac{3}{2}$ currents $G^{ \pm, a}$ remain the same when we select the right choice for the index $a$ as $S U(2)$. The previous indices $1,2,3$ in (8.1) correspond to $1,4,7$ for $M=3$ case. From the definition of $G^{ \pm, a}(2.11)$, the summations over $j$ and $\bar{i}$ contain the index 3 . However, any generators $t^{a}$ for indices $a=1,4,7$ do not have any rows and columns having an index 3 . Then we are left with the spin- $\frac{3}{2}$ currents $G^{ \pm, a}$ with same field contents for $M=2$ case. Now we look at the other spin- $\frac{3}{2}$ currents in $G^{ \pm}(2.7)$. In this case, there exist the summations over $j$ and $\bar{i}$ having the index 3 . Therefore, as we increase the $M$ value, the field contents for these spin- $\frac{3}{2}$ currents are increasing. In other words, the field contents of spin- $\frac{3}{2}$ currents for $M=3$ are the same as the one for $M=2$ and other terms.

This implies that for example, the OPE between $\hat{G}_{11}(z)$ and $\hat{G}_{12}$, where the numerical factor 2 inside of the square root is replaced by 3 and the indices 1, 2, 3 are replaced by $1,4,7$, does not lead to the one of the known relation in the large $\mathcal{N}=4$ nonlinear superconformal algebra due to the

\footnotetext{
$\overline{37}$ In the next lowest $\mathcal{N}=4$ multiplet, there are also other four spin- $\frac{5}{2}$ currents.
}

contribution from other terms we mentioned above. Therefore, we do not have $\mathcal{N}=4$ supersymmetry for $M=3$ and we expect that this holds for $M>2$.

In summary, the currents of $\mathcal{N}=4$ nonlinear superconformal algebra are given by (8.1) together with other currents appearing in the footnotes 35 and 36. Moreover, the higher spin- $\frac{3}{2}, 2$ currents are described in (8.2) and (8.3). The spin$\frac{5}{2}$ currents are in Appendix $\mathrm{H}$ for fixed $N=5$. Finally, the spin-3 current is given by (8.4) implicitly.

\section{Conclusions and outlook}

The highest component of the first $\mathcal{N}=2$ multiplet is found in (3.1). The second $\mathcal{N}=2$ multiplet is found by (4.7), (4.5), (4.6) and (4.11). The third $\mathcal{N}=2$ multiplet is obtained from (5.7), (5.9) and (5.11). Their OPEs between the currents of the $\mathcal{N}=2$ superconformal algebra and these above currents are determined completely in the Sects. 3, 4 and 5. Moreover the OPEs between the first $\mathcal{N}=2$ multiplet and itself are described with the observation of three kinds of new primary operators. Similarly, we describe the OPE between the lowest singlet spin-2 current and itself in the second $\mathcal{N}=2$ multiplet. Finally, the extension of the large $\mathcal{N}=4$ nonlinear superconformal algebra for $M=2$ is realized from the coset fields living in (1.2).

Therefore, we have obtained some currents (and their OPEs) in the supersymmetric coset model for generic $k, N$ and $M$ which correspond to the generators of the $\mathcal{N}=2$ "rectangular" $W$-algebra in the $A d S_{3}$ bulk theory according to a holography [30]. We have obtained the $\mathcal{N}=2$ version of [12] and obtained the generalization of [72] (which is for $M=2$ case) to a generic $M$. In this paper, we have $f, d$ symbols or Kronecker delta in the structure constants of the OPEs compared with the ones of [72] with various invariant tensors. For generic $M>2$, we do not have to worry about the identities between these invariant tensors although the supersymmetry is given by $\mathcal{N}=2$.

We present the future directions related to the results of this paper as follows:

\section{- More supersymmetric cases}

It is obvious that when we further restrict the level for $S U(N+M)_{k}$ to be the dual Coxeter number of $S U(N+M)$, there arises an enhancement of the $\mathcal{N}=3$ supersymmetry [82]. It would be interesting to see how we can construct the relevant coset fields explicitly by using the additional fermions. Note that in the coset of (1.2), there is no $S U(M)_{k+N}$ factor in the denominator and this will lead to more general case because we should add this factor into the one of [82]. 
- At the critical level with $M=2$

In this case (by fixing the $M$ value further in previous consideration), we should check whether the original $\mathcal{N}=4$ supersymmetry is enhanced to more supersymmetric cases or not. The point is how to obtain the additional supersymmetry generator by using the additional spin- $\frac{1}{2}$ fermions without spoiling the present $\mathcal{N}=4$ supersymmetry generators.

- More OPEs between the $\mathcal{N}=2$ multiplets

So far we have considered the simplest OPEs in Sect. 6. However, there are other OPEs between the $\mathcal{N}=2$ multiplets we should consider. It is an open problem to study their OPEs systematically.

- Are there any $\mathcal{N}=2$ primary basis?

We have seen that the $\mathcal{N}=2$ superconformal algebra we describe in this paper has the modified stress energy tensor. It is an open problem to find whether there are any $\mathcal{N}=2$ primary basis where the description of Appendix B is satisfied or not. What happens if we use the modified stress energy tensor rather than the stress energy tensor $T(2.5)$ ?

- How do we construct the higher spin algebra in the $A d S_{3}$ gravity side via holography?

According to the result of [3] on the holography, it is natural to ask whether we can construct the corresponding $\mathcal{N}=2$ higher spin algebra in the supersymmetric coset model in this paper. For $M=2$, there is a construction in [70]. For generic $M$, it is an interesting problem to obtain them explicitly. The additional $M$ dependence will play the role newly.

- The orthogonal case

Once we have found the higher spin currents in the bosonic orthogonal case, then its supersymmetric version can be obtained similarly. If we use the embedding between the unitary and orthogonal groups, then maybe the $f$ and $d$ symbols in the unitary group can be used. The previous works [83-86] can be used. See also [87].

- Are there any systematical construction for the currents having more than two free indices?

We have seen the new primary currents in the Sect. 6 having the two free indices. Then the question is how we can determine the spin contents of this kind of primary currents. As a first step, we should obtain the $\mathcal{N}=2$ description for the above three spin- $\frac{5}{2}, 3$ currents. That is, can we obtain the possible lowest (and highest) components explicitly?
- Extension of the large $\mathcal{N}=4$ linear superconformal algebra

We can think of the large $\mathcal{N}=4$ linear superconformal algebra [88-96] by introducing the four spin- $\frac{1}{2}$ operators and the spin- 1 current with fixed $M=2$. For the spin- 1 current we can think of some linear combination of the spin-1 operator $J^{u(1)}$ and $J_{f}^{u(1)}$ appearing in the spin-1 current of (2.9) as a candidate. For the spin- $\frac{1}{2}$ operators, it is not clear how to construct them by some contractions of the indices. It would be interesting to study further.

Acknowledgements We thank Y. Hikida for the discussions. This work was supported by the National Research Foundation of Korea (NRF) Grant funded by the Korea government (MSIT) (No. 2020R1F1A1066 893).

Data Availability Statement This manuscript has no associated data or the data will not be deposited. [Authors' comment: All data generated or analysed during this study are included in this published article [and its supplementary information files].]

Open Access This article is licensed under a Creative Commons Attribution 4.0 International License, which permits use, sharing, adaptation, distribution and reproduction in any medium or format, as long as you give appropriate credit to the original author(s) and the source, provide a link to the Creative Commons licence, and indicate if changes were made. The images or other third party material in this article are included in the article's Creative Commons licence, unless indicated otherwise in a credit line to the material. If material is not included in the article's Creative Commons licence and your intended use is not permitted by statutory regulation or exceeds the permitted use, you will need to obtain permission directly from the copyright holder. To view a copy of this licence, visit http://creativecomm ons.org/licenses/by/4.0/.

Funded by $\mathrm{SCOAP}^{3}$.

\section{A The $\mathcal{N}=2$ superconformal algebra with a modified stress energy tensor}

In this Appendix, we describe the $\mathcal{N}=2$ superconformal algebra discussed in Sect. 2.

A.1 The OPEs between the spin- $\frac{1}{2}$ operators and the spin-1 operators

By using (2.2) and (2.3) we obtain

$$
\begin{aligned}
& \psi^{(\rho \bar{i})}(z) J_{f}^{\alpha}(w)=-\frac{1}{(z-w)} t_{\sigma \bar{\tau}}^{\alpha} \delta^{\rho \bar{\tau}} \psi^{(\sigma \bar{i})}(w)+\cdots, \\
& \psi^{(\bar{\sigma} j)}(z) J_{f}^{\alpha}(w)=\frac{1}{(z-w)} t_{\tau \bar{v}}^{\alpha} \delta^{\tau \bar{\sigma}} \psi^{(\bar{v} j)}(w)+\cdots, \\
& \psi^{(\rho \bar{i})}(z) J_{f}^{a}(w)=\frac{1}{(z-w)} t_{l \bar{k}}^{a} \delta^{l \bar{i}} \psi^{(\rho \bar{k})}(w)+\cdots, \\
& \psi^{(\bar{\sigma} j)}(z) J_{f}^{a}(w)=-\frac{1}{(z-w)} t_{l \bar{k}}^{a} \delta^{j \bar{k}} \psi^{(\bar{\sigma} l)}(w)+\cdots,
\end{aligned}
$$


$\psi^{(\rho \bar{i})}(z) J_{f}^{u(1)}(w)=-\frac{1}{(z-w)} \psi^{(\rho \bar{i})}(w)+\cdots$,

$\psi^{(\bar{\sigma} j)}(z) J_{f}^{u(1)}(w)=\frac{1}{(z-w)} \psi^{(\bar{\sigma} j)}(w)+\cdots$.

\section{A.2 The OPEs between the spin-1 operators}

Similarly, from the relations (2.2) and (2.3), the following OPEs satisfy

$$
\begin{aligned}
& J_{f}^{\alpha}(z) J_{f}^{\beta}(w)=\frac{1}{(z-w)^{2}} M \delta^{\alpha \beta} \\
& +\frac{1}{(z-w)} i f^{\alpha \beta \gamma} J_{f}^{\gamma}(w)+\cdots, \\
& J_{f}^{\alpha}(z) J_{f}^{a}(w)=0+\cdots, \\
& J_{f}^{\alpha}(z) J_{f}^{u(1)}(w)=0+\cdots, \\
& J_{f}^{a}(z) J_{f}^{b}(w)=\frac{1}{(z-w)^{2}} N \delta^{a b} \\
& +\frac{1}{(z-w)} i f^{a b c} J_{f}^{c}(w)+\cdots, \\
& J_{f}^{a}(z) J_{f}^{u(1)}(w)=0+\cdots, \\
& J_{f}^{u(1)}(z) J_{f}^{u(1)}(w)=\frac{1}{(z-w)^{2}} M N+\cdots .
\end{aligned}
$$

We can read off various levels in these OPEs.

A.3 The OPEs between the spin- $\frac{1}{2}$ operators and the currents of $\mathcal{N}=2$ superconformal algebra

By using (2.5), (2.7), (2.8) and (2.2) we determine the following OPEs

$$
\begin{aligned}
& \psi^{(\rho \bar{i})}(z) K(w)=\frac{1}{(z-w)} \frac{k}{(k+M+N)} \psi^{(\rho \bar{i})}(w)+\cdots, \\
& \psi^{(\bar{\sigma} j)}(z) K(w)=-\frac{1}{(z-w)} \frac{k}{(k+M+N)} \psi^{(\bar{\sigma} j)}(w)+\cdots, \\
& \psi^{(\rho \bar{i})}(z) G^{+}(w)=\frac{1}{(z-w)} J^{(\rho \bar{i})}(w)+\cdots, \\
& \psi^{(\bar{\sigma} j)}(z) G^{+}(w)=0+\cdots, \\
& \psi^{(\rho \bar{i})}(z) G^{-}(w)=0+\cdots, \\
& \psi^{(\bar{\sigma} j)}(z) G^{-}(w)=\frac{1}{(z-w)} J^{(\bar{\sigma} j)}(w)+\cdots, \\
& \psi^{(\rho \bar{i})}(z) T(w)=\frac{1}{(z-w)^{2}} \frac{\left(k M+M^{2}-1\right)}{2 M(k+M+N)} \psi^{(\rho \bar{i})}(w) \\
& +\frac{1}{(z-w)}\left[\frac{1}{(k+M+N)} t_{\sigma \bar{\tau}}^{\alpha} \delta^{\rho \bar{\tau}} J^{\alpha} \psi^{(\sigma \bar{i})}\right. \\
& +\frac{1}{(k+M+N)} t_{\sigma \bar{\tau}}^{\alpha} \delta^{\rho \bar{\tau}} \psi^{(\bar{\sigma} i)} J_{f}^{\alpha} \\
& +\frac{1}{(k+M+N)} \sqrt{\frac{M+N}{M N}} J^{u(1)} \psi^{(\bar{\sigma} j)}
\end{aligned}
$$

$$
\begin{aligned}
& +\frac{1}{(k+M+N)} \frac{(M+N)}{M N} \psi^{(\rho \bar{i})} J_{f}^{u(1)} \\
& \left.-\frac{\left(k M+M^{2}-1\right)}{2 M(k+M+N)} \partial \psi^{(\rho \bar{i})}\right](w)+\cdots, \\
& \psi^{(\bar{\sigma} j)}(z) T(w)=\frac{1}{(z-w)^{2}} \frac{\left(k M+M^{2}-1\right)}{2 M(k+M+N)} \psi^{(\bar{\sigma} j)}(w) \\
& +\frac{1}{(z-w)}\left[-\frac{1}{(k+M+N)} t_{\tau \bar{\nu}}^{\alpha} \delta^{\tau \bar{\sigma}} J^{\alpha} \psi^{(\bar{v} j)}\right. \\
& -\frac{1}{(k+M+N)} t_{\tau \bar{v}}^{\alpha} \delta^{\tau \bar{\sigma}} \psi^{(\bar{v} j)} J_{f}^{\alpha} \\
& -\frac{1}{(k+M+N)} \sqrt{\frac{M+N}{M N}} J^{u(1)} \psi^{(\bar{\sigma} j)} \\
& -\frac{1}{(k+M+N)} \frac{(M+N)}{M N} \psi^{(\bar{\sigma} j)} J_{f}^{u(1)} \\
& \left.-\frac{\left(k M+M^{2}-1\right)}{2 M(k+M+N)} \partial \psi^{(\bar{\sigma} j)}\right](w)+\cdots .
\end{aligned}
$$

A.4 The OPEs between the spin-1 operators and the currents of $\mathcal{N}=2$ superconformal algebra

We have some OPEs between the spin-1 operators and the currents of $\mathcal{N}=2$ superconformal algebra as follows:

$$
\begin{aligned}
& J_{f}^{u(1)}(z) K(w)=-\frac{1}{(z-w)^{2}} \frac{k M N}{(k+M+N)}+\cdots, \\
& J_{f}^{u(1)}(z) G^{+}(w)=-\frac{1}{(z-w)} G^{+}+\cdots, \\
& J_{f}^{u(1)}(z) G^{-}(w)=\frac{1}{(z-w)} G^{-}+\cdots,
\end{aligned}
$$$$
J_{f}^{u(1)}(z) T(w)=-\frac{1}{(z-w)^{2}} K(w)+\cdots,
$$$$
J^{u(1)}(z) K(w)=\frac{1}{(z-w)^{2}} \frac{k M N}{(k+M+N)} \sqrt{\frac{M+N}{M N}}+\cdots,
$$$$
J^{u(1)}(z) G^{+}(w)=\frac{1}{(z-w)} \sqrt{\frac{M+N}{M N}} G^{+}+\cdots,
$$$$
J^{u(1)}(z) G^{-}(w)=-\frac{1}{(z-w)} \sqrt{\frac{M+N}{M N}} G^{-}+\cdots,
$$$$
J^{u(1)}(z) T(w)=\frac{1}{(z-w)^{2}} \sqrt{\frac{M+N}{M N}} K(w)+\cdots,
$$$$
J^{(\rho \bar{i})}(z) K(w)=-\frac{1}{(z-w)} \frac{(M+N)}{(k+M+N)} J^{(\rho \bar{i})}(w)+\cdots,
$$$$
J^{(\rho \bar{i})}(z) G^{+}(w)=0+\cdots,
$$$$
J^{(\rho \bar{i})}(z) G^{-}(w)=\frac{1}{(z-w)^{2}} k \psi^{(\rho \bar{i})}(w)
$$$$
+\frac{1}{(z-w)}\left[\sqrt{\frac{M+N}{M N}} \psi^{(\rho \bar{i})} J^{u(1)}+t_{\sigma \bar{\rho}}^{\alpha} \delta^{\rho \bar{\rho}} \psi^{(\sigma \bar{i})} J^{\alpha}\right.
$$$$
\left.-t_{i \bar{k}}^{a} \delta^{i \bar{i}} \psi^{(\rho \bar{k})} J^{a}\right](w)+\cdots
$$

$J^{(\rho \bar{i})}(z) T(w)=\frac{1}{(z-w)^{2}}\left[\frac{2 k M+2 M^{2}+M N-1}{2 M(k+M+N)}\right] J^{(\rho \bar{i})}(w)$ 


$$
\begin{aligned}
& +\frac{1}{(z-w)}\left[\frac{1}{(k+M+N)} \sqrt{\frac{M+N}{M N}} J^{(\rho \bar{i})} J^{u(1)}\right. \\
& +\frac{(M+N)}{M N(k+M+N)} J^{(\rho \bar{i})} J_{f}^{u(1)} \\
& +\frac{1}{(k+M+N)} t_{\sigma \bar{\rho}}^{\alpha} \delta^{\rho \bar{\rho}} J^{\alpha} J^{(\sigma \bar{i})} \\
& +\frac{1}{(k+M+N)} t_{\sigma \bar{\rho}}^{\alpha} \delta^{\rho \bar{\rho}} J^{(\sigma \bar{i})} J_{f}^{\alpha} \\
& \left.-\frac{\left(M N^{2}-2 M-N\right)}{2 M N(k+M+N)} \partial J^{(\rho \bar{i})}\right](w)+\cdots, \\
& J^{(\bar{\sigma} j)}(z) K(w)=\frac{1}{(z-w)} \frac{(M+N)}{(k+M+N)} J^{(\bar{\sigma} j)}(w)+\cdots, \\
& J^{(\bar{\sigma} j)}(z) G^{+}(w)=\frac{1}{(z-w)^{2}} k \psi^{(\bar{\sigma} j)}(w) \\
& +\frac{1}{(z-w)}\left[-\sqrt{\frac{M+N}{M N}} \psi^{(\bar{\sigma} j)} J^{u(1)}\right. \\
& \left.-t_{\rho \bar{\tau}}^{\alpha} \delta^{\rho \bar{\sigma}} \psi^{(\bar{\tau} j)} J^{\alpha}+t_{k \bar{k}}^{a} \delta^{j \bar{k}} \psi^{(\bar{\sigma} k)} J^{a}\right](w)+\cdots, \\
& J^{(\bar{\sigma} j)}(z) G^{-}(w)=0+\cdots, \\
& J^{(\bar{\sigma} j)}(z) T(w)=\frac{1}{(z-w)^{2}}\left[\frac{2 k M+2 M^{2}+M N-1}{2 M(k+M+N)}\right] J^{(\bar{\sigma} j)}(w) \\
& +\frac{1}{(z-w)}\left[-\frac{1}{(k+M+N)} \sqrt{\frac{M+N}{M N}} J^{(\bar{\sigma} j)} J^{u(1)}\right. \\
& -\frac{(M+N)}{M N(k+M+N)} J^{(\bar{\sigma} j)} J_{f}^{u(1)} \\
& -\frac{1}{(k+M+N)} t_{\sigma \bar{\tau}}^{\alpha} \delta^{\sigma \bar{\sigma}} J^{\alpha} J^{(\bar{\tau} j)} \\
& -\frac{1}{(k+M+N)} t_{\sigma \bar{\tau}}^{\alpha} \delta^{\sigma \bar{\sigma}} J^{(\bar{\tau} j)} J_{f}^{\alpha} \\
& \left.-\frac{\left(M N^{2}-2 M-N\right)}{2 M N(k+M+N)} \partial J^{(\bar{\sigma} j)}\right](w)+\cdots, \\
& J_{f}^{a}(z) K(w)=0+\cdots, \\
& J_{f}^{a}(z) G^{+}(w)=-\frac{1}{(z-w)} G^{+, a}(w)+\cdots, \\
& J_{f}^{a}(z) G^{-}(w)=\frac{1}{(z-w)} G^{-, a}(w)+\cdots, \\
& J_{f}^{a}(z) T(w)=\frac{1}{(z-w)^{2}} J_{f}^{a}(w)+\cdots, \\
& J_{f}^{\alpha}(z) K(w)=0+\cdots, \\
& J_{f}^{\alpha}(z) G^{+}(w)=-\frac{1}{(z-w)} t_{\rho \bar{v}}^{\alpha} \delta_{j \bar{i}} J^{(\rho \bar{i})} \psi^{(\bar{v} j)}(w)+\cdots, \\
& J_{f}^{\alpha}(z) G^{-}(w)=\frac{1}{(z-w)} t_{\sigma \bar{\sigma}}^{\alpha} \delta_{j \bar{i}} \\
& \psi^{(\sigma \bar{i})} J^{(\bar{\sigma} j)}(w)+\cdots, \\
& J_{f}^{\alpha}(z) T(w)=\frac{1}{(z-w)^{2}} \frac{1}{(k+M+N)} \\
& \times\left[k J_{f}^{\alpha}-M J^{\alpha}\right](w) \\
& -\frac{1}{(z-w)} \frac{1}{(k+M+N)} i f^{\alpha \beta \gamma} J^{\beta} J_{f}^{\gamma}(w)+\cdots \text {. }
\end{aligned}
$$

We can calculate the following OPEs

$$
\begin{aligned}
& J_{f}^{u(1)}(z) \delta_{\rho \bar{\sigma}} \delta_{j \bar{i}} \psi^{(\rho \bar{i})} \partial \psi^{(\bar{\sigma} j)}(w) \\
& =\frac{1}{(z-w)^{3}} M N-\frac{1}{(z-w)^{2}} J_{f}^{u(1)}(w)+\cdots, \\
& J_{f}^{u(1)}(z) \delta_{\rho \bar{\sigma}} \delta_{j \bar{i}} \partial \psi^{(\rho \bar{i})} \psi^{(\bar{\sigma} j)}(w) \\
& =\frac{1}{(z-w)^{3}} M N+\frac{1}{(z-w)^{2}} J_{f}^{u(1)}(w)+\cdots, \\
& J_{f}^{u(1)}(z) J_{f}^{u(1)} J_{f}^{u(1)}(w) \\
& =\frac{1}{(z-w)^{2}} 2 M N J_{f}^{u(1)}(w)+\cdots .
\end{aligned}
$$

A.5 The $\mathcal{N}=2$ superconformal algebra

As before, we determine the following OPEs from (2.5), (2.7) and (2.8)

$$
\begin{aligned}
& K(z) K(w)=\frac{1}{(z-w)^{2}} \frac{k M N}{(k+M+N)}+\cdots, \\
& K(z) G^{+}(w)=\frac{1}{(z-w)} G^{+}(w)+\cdots, \\
& K(z) G^{-}(w)=-\frac{1}{(z-w)} G^{-}(w)+\cdots, \\
& K(z) T(w)=\frac{1}{(z-w)^{2}} K(w)+\cdots, \\
& G^{+}(z) G^{+}(w)=0+\cdots, \\
& G^{+}(z) G^{-}(w)=\frac{1}{(z-w)^{3}} k M N \\
& +\frac{1}{(z-w)^{2}}(k+M+N) K(w) \\
& +\frac{1}{(z-w)}\left[\frac{1}{2}(k+M+N) \partial K\right. \\
& +(k+M+N)\left(T-\frac{1}{2(k+M+N)}\right. \\
& \left.\left.\left(J^{a}+J_{f}^{a}\right)\left(J^{a}+J_{f}^{a}\right)\right)\right](w)+\cdots, \\
& G^{+}(z) T(w)=\frac{1}{(z-w)^{2}} \frac{3}{2} G^{+}(w) \\
& +\frac{1}{(z-w)} \frac{1}{2} \partial G^{+}(w)+\cdots, \\
& G^{-}(z) T(w)=\frac{1}{(z-w)^{2}} \frac{3}{2} G^{-}(w) \\
& +\frac{1}{(z-w)} \frac{1}{2} \partial G^{-}(w)+\cdots, \\
& T(z) T(w)=\frac{1}{(z-w)^{4}} \frac{1}{2} \frac{\left(k M^{2}+3 k M N-k+M^{2} N-N\right)}{(k+M+N)} \\
& +\frac{1}{(z-w)^{2}} 2 T(w)+\frac{1}{(z-w)} \partial T(w)+\cdots .
\end{aligned}
$$

The central term in the OPE of $K(z) K(w)$ is the same as the standard expression $\frac{c}{3}$ for only $M=1$ value. We can multiply $\frac{1}{(k+M+N)}$ in the OPE of $G^{+}(z) G^{-}(w)$ in order to have the same central term as the one of the OPE of $K(z) K(w)$. 
In obtaining the above result, we observe that there exist the relations

$$
\begin{aligned}
& J_{f}^{\alpha} J_{f}^{\alpha}=t_{\rho \bar{\sigma}}^{\alpha} t_{\tau \bar{\nu}}^{\alpha} \delta_{j \bar{i}} \delta_{l \bar{k}} \psi^{(\rho \bar{i})} \psi^{(\bar{\sigma} j)} \psi^{(\tau \bar{k})} \psi^{(\bar{v} l)} \\
& +\frac{\left(N^{2}-1\right)}{N} \delta_{\rho \bar{\sigma}} \delta_{j \bar{i}} \partial \psi^{(\rho \bar{i})} \psi^{(\bar{\sigma} j)} \\
& \quad-\frac{\left(N^{2}-1\right)}{N} \delta_{\rho \bar{\sigma}} \delta_{j \bar{i}} \psi^{(\rho \bar{i})} \partial \psi^{(\bar{\sigma} j)}, \\
& J_{f}^{u(1)} J_{f}^{u(1)}=\delta_{\rho \bar{\sigma}} \delta_{\tau \bar{\nu}} \delta_{j \bar{i}} \delta_{l \bar{k}} \psi^{(\rho \bar{i})} \psi^{(\bar{\sigma} j)} \psi^{(\tau \bar{k})} \psi^{(\bar{v} l)} \\
& +\delta_{\rho \bar{\sigma}} \delta_{j \bar{i}} \partial \psi^{(\rho \bar{i})} \psi^{(\bar{\sigma} j)} \\
& \quad-\delta_{\rho \bar{\sigma}} \delta_{j \bar{i}} \psi^{(\rho \bar{i})} \partial \psi^{(\bar{\sigma} j)}, \\
& J_{f}^{a} J_{f}^{a}=t_{j \bar{i}}^{a} t_{l \bar{k}}^{a} \delta_{\rho \bar{\sigma}} \delta_{\tau \bar{\nu}} \psi^{(\rho \bar{i})} \psi^{(\bar{\sigma} j)} \psi^{(\tau \bar{k})} \psi^{(\bar{v} l)} \\
& +\frac{\left(M^{2}-1\right)}{M} \delta_{\rho \bar{\sigma}} \delta_{j \bar{i}} \partial^{(\rho \bar{i})} \psi^{(\bar{\sigma} j)} \\
& \quad-\frac{\left(M^{2}-1\right)}{M} \delta_{\rho \bar{\sigma}} \delta_{j \bar{i}} \psi^{(\rho \bar{i})} \partial \psi^{(\bar{\sigma} j)} .
\end{aligned}
$$

We can check the following identity between the spin-1 currents in (2.3) by using (A.1)

$$
\begin{aligned}
& J_{f}^{\alpha} J_{f}^{\alpha}+\left(\frac{1}{M}+\frac{1}{N}\right) J_{f}^{u(1)} J_{f}^{u(1)}+J_{f}^{a} J_{f}^{a}= \\
& (M+N)\left(\delta_{\rho \bar{\sigma}} \delta_{j \bar{i}} \partial \psi^{(\rho \bar{i})} \psi^{(\bar{\sigma} j)}-\delta_{\rho \bar{\sigma}} \delta_{j \bar{i}} \psi^{(\rho \bar{i})} \partial \psi^{(\bar{\sigma} j)}\right) .
\end{aligned}
$$

\section{B The $\mathcal{N}=2$ primary conditions}

The $\mathcal{N}=2$ primary conditions for the currents $\left(\Phi^{-(h)}\right.$, $\left.\Phi^{+\left(h+\frac{1}{2}\right)}, \Phi^{-\left(h+\frac{1}{2}\right)}, \Phi^{+(h+1)}\right)$ are summarized by

$K(z) \Phi^{-(h)}(w)=0+\cdots$,

$K(z) \Phi^{+\left(h+\frac{1}{2}\right)}(w)=\frac{1}{(z-w)} \Phi^{+\left(h+\frac{1}{2}\right)}(w)+\cdots$,

$K(z) \Phi^{-\left(h+\frac{1}{2}\right)}(w)=-\frac{1}{(z-w)} \Phi^{-\left(h+\frac{1}{2}\right)}(w)+\cdots$,

$K(z) \Phi^{+(h+1)}(w)=\frac{1}{(z-w)^{2}} h \Phi^{-(h)}(w)+\cdots$,

$G^{+}(z) \Phi^{-(h)}(w)=-\frac{1}{(z-w)} \Phi^{+\left(h+\frac{1}{2}\right)}(w)+\cdots$,

$G^{+}(z) \Phi^{+\left(h+\frac{1}{2}\right)}(w)=0+\cdots$,

$G^{+}(z) \Phi^{-\left(h+\frac{1}{2}\right)}(w)=\frac{1}{(z-w)^{2}} h \Phi^{-(h)}(w)$

$+\frac{1}{(z-w)}\left[\Phi^{+(h+1)}+\frac{1}{2} \partial \Phi^{-(h)}\right](w)+\cdots$,

$G^{+}(z) \Phi^{+(h+1)}(w)=\frac{1}{(z-w)^{2}}\left(h+\frac{1}{2}\right) \Phi^{+\left(h+\frac{1}{2}\right)}(w)$

$+\frac{1}{(z-w)} \frac{1}{2} \partial \Phi^{+\left(h+\frac{1}{2}\right)}(w)+\cdots$,

$G^{-}(z) \Phi^{-(h)}(w)=\frac{1}{(z-w)} \Phi^{-\left(h+\frac{1}{2}\right)}(w)+\cdots$,

$$
\begin{aligned}
& G^{-}(z) \Phi^{+\left(h+\frac{1}{2}\right)}(w)=-\frac{1}{(z-w)^{2}} h \Phi^{-(h)}(w) \\
& +\frac{1}{(z-w)}\left[\Phi^{+(h+1)}-\frac{1}{2} \partial \Phi^{-(h)}\right](w)+\cdots, \\
& G^{-}(z) \Phi^{-\left(h+\frac{1}{2}\right)}(w)=0+\cdots, \\
& G^{-}(z) \Phi^{+(h+1)}(w)=\frac{1}{(z-w)^{2}}\left(h+\frac{1}{2}\right) \Phi^{-\left(h+\frac{1}{2}\right)}(w) \\
& +\frac{1}{(z-w)} \frac{1}{2} \partial \Phi^{-\left(h+\frac{1}{2}\right)}(w)+\cdots, \\
& T(z) \Phi^{-(h)}(w)=\frac{1}{(z-w)^{2}} h \Phi^{-(h)}(w) \\
& +\frac{1}{(z-w)} \partial \Phi^{-(h)}(w)+\cdots, \\
& T(z) \Phi^{+\left(h+\frac{1}{2}\right)}(w)=\frac{1}{(z-w)^{2}}\left(h+\frac{1}{2}\right) \Phi^{+\left(h+\frac{1}{2}\right)}(w) \\
& +\frac{1}{(z-w)} \partial \Phi^{+\left(h+\frac{1}{2}\right)}(w)+\cdots, \\
& T(z) \Phi^{-\left(h+\frac{1}{2}\right)}(w)=\frac{1}{(z-w)^{2}}\left(h+\frac{1}{2}\right) \Phi^{-\left(h+\frac{1}{2}\right)}(w) \\
& +\frac{1}{(z-w)} \partial \Phi^{-\left(h+\frac{1}{2}\right)}(w)+\cdots, \\
& T(z) \Phi^{+(h+1)}(w)=\frac{1}{(z-w)^{2}}(h+1) \Phi^{+(h+1)}(w) \\
& +\frac{1}{(z-w)} \partial \Phi^{+(h+1)}(w)+\cdots .
\end{aligned}
$$

In this paper, we observe that the currents we are considering do not satisfy these OPEs. That is, some of the higher order terms arise and the structure constants appear differently.

\section{Some OPEs between the spin-1 currents and the singlet and nonsinglet spin-3 currents in Sects. 4 and 5}

In order to obtain the OPEs between the supersymmetry generators and the nonsinglet and singlet spin-3 currents obtained in the bosonic coset model, we need to calculate the following OPEs between the spin-1 currents and the singlet and nonsinglet spin-3 currents.

C.1 The OPE between $J^{(\rho \bar{i})}(z) W^{(3)}(w)$

The OPE between the spin- 1 current and the spin-3 current is summarized by

$$
\begin{aligned}
& J^{(\rho \bar{i})}(z) W^{(3)}(w)=-\frac{1}{(z-w)^{3}} \\
& \frac{\left(k^{2}-1\right)\left(k^{2}-4\right)(k+M+N)(2 k+M+N)(3 k+2 M+2 N)}{k^{2} M(k+M)(k+2 M)} \\
& \quad b_{1} J^{(\rho \bar{i})}(w)+\frac{1}{(z-w)^{2}} \frac{\left(k^{2}-4\right)(k+M+N)(3 k+2 M+2 N)}{k M(k+2 M)} b_{1}
\end{aligned}
$$




$$
\begin{aligned}
& \times\left[-\sqrt{\frac{M+N}{M N}} \frac{3(k+N)}{k} J^{u(1)} J^{(\rho \bar{i})}\right. \\
& +\frac{3(k+N)}{(k+M)} t_{i \bar{k}}^{a} \delta^{i \bar{i}} J^{a} J^{(\rho \bar{k})}-3 t_{\sigma \bar{\rho}}^{\alpha} \delta^{\rho \bar{\rho}} J^{\alpha} J^{(\sigma \bar{i})} \\
& \left.+\frac{\left(k^{3}+2 k^{2} M+2 k^{2} N+3 k M N+2 k+M+N\right)}{k(k+M)} \partial J^{(\rho \bar{i})}\right](w) \\
& +\frac{1}{(z-w)} \frac{(k+M+N)}{M} b_{1} \\
& \times\left[-3 d^{\alpha \beta \gamma} t_{\sigma \bar{\rho}}^{\gamma} \delta^{\rho \bar{\rho}} J^{\alpha} J^{\beta} J^{(\sigma \bar{i})}\right. \\
& -\frac{3(k+N)(k+2 N)}{(k+M)(k+2 M)} d^{a b c} t_{i \bar{k}}^{c} \delta^{i \bar{i}} J^{a} J^{b} J^{(\rho \bar{k})} \\
& -\frac{6(k+N)(k+2 N)(M+N)}{k^{2} M N} J^{u(1)} J^{u(1)} J^{(\rho \bar{i})} \\
& -\frac{6(k+N)}{k N} J^{\alpha} J^{\alpha} J^{(\rho \bar{i})} \\
& -\sqrt{\frac{M+N}{M N}} \frac{12(k+N)}{k} t_{\sigma \bar{\sigma}}^{\alpha} \delta^{\rho \bar{\sigma}} J^{\alpha} J^{u(1)} J^{(\sigma \bar{i})} \\
& -\frac{6(k+N)(k+2 N)}{k M(k+2 M)} J^{a} J^{a} J^{(\rho \bar{i})} \\
& +\sqrt{\frac{M+N}{M N}} \frac{12(k+N)(k+2 N)}{k(k+2 M)} t_{i \bar{k}}^{a} \delta^{i \bar{i}} J^{a} J^{u(1)} J^{(\rho \bar{k})} \\
& +\frac{12(k+N)}{(k+2 M)} \delta_{\nu \bar{\tau}} \delta_{k \bar{j}} J^{(\nu \bar{i})} J^{(\rho \bar{j})} J^{(\bar{\tau} k)} \\
& +\frac{24(k+N)}{k(k+2 M)} \delta_{\sigma \bar{\tau}} \delta_{k \bar{j}} J^{(\rho \bar{i})} J^{(\sigma \bar{j})} J^{(\bar{\tau} k)} \\
& -\sqrt{\frac{M+N}{M N}} \frac{12(k+N)}{k} t_{\sigma \bar{\rho}}^{\alpha} \delta^{\rho \bar{\rho}} J^{\alpha} J^{u(1)} J^{(\sigma \bar{j})} \\
& +\frac{12(k+N)}{(k+2 M)} t_{i \bar{j}}^{a} \delta^{i \bar{i}} t_{\sigma \bar{\rho}}^{\alpha} \delta^{\rho \bar{\rho}} J^{\alpha} J^{a} J^{(\sigma \bar{j})} \\
& +\frac{3\left(k^{2}+2 k N+4\right)}{k} t_{\tau \bar{\rho}}^{\alpha} \delta^{\rho \bar{\rho}} J^{\alpha} \partial J^{(\tau \bar{i})} \\
& -\frac{3\left(k^{2}+2 k M+4\right)(k+N)(k+2 N)}{k(k+M)(k+2 M)} t_{i \bar{l}}^{a} \delta^{i \bar{i}} J^{a} \partial J^{(\rho \bar{l})}+\sqrt{\frac{M+N}{M N}} \\
& , \times \frac{3(k+N)\left(k^{3}+2 k^{2} M+2 k^{2} N+8 k M N+4 k+8 M+8 N\right)}{k^{2}(k+2 M)} \\
& \times J^{u(1)} \partial J^{(\rho \bar{i})}-3(k+N) t_{\tau \bar{\rho}}^{\alpha} \delta^{\rho \bar{\rho}} \partial J^{\alpha} J^{(\tau \bar{i})} \\
& +\frac{3(k+N)(k+2 N)}{(k+2 M)} t_{i \bar{j}}^{a} \delta^{i \bar{i}} \partial J^{a} J^{(\rho \bar{j})} \\
& -\sqrt{\frac{M+N}{M N}} \frac{3(k+N)(k+2 N)}{k} \partial J^{u(1)} J^{(\rho \bar{i})} \\
& -\frac{\left(k^{2}-4\right)}{2 k^{2}(k+M)(k+2 M)}\left(k^{3} M+k^{3} N+2 k^{2} M^{2}\right. \\
& -2 k^{2} M N+2 k^{2} N^{2}-6 k^{2}-7 k M \\
& \left.\left.-7 k N-2 M^{2}-4 M N-2 N^{2}\right) \partial^{2} J^{(\rho \bar{i})}\right](w)+\cdots .
\end{aligned}
$$

C.2 The OPE between $J^{(\bar{\sigma} j)}(z) W^{(3)}(w)$

Similarly, the OPE between the other spin-1 current and the spin-3 current is summarized by

$$
\begin{aligned}
& J^{(\bar{\sigma} j)}(z) W^{(3)}(w)=\frac{1}{(z-w)^{3}} \\
& \underline{\left(k^{2}-1\right)\left(k^{2}-4\right)(k+M+N)(2 k+M+N)(3 k+2 M+2 N)} \\
& k^{2} M(k+M)(k+2 M) \\
& b_{1} J^{(\bar{\sigma} j)}(w)+\frac{1}{(z-w)^{2}} \frac{\left(k^{2}-4\right)(k+M+N)(3 k+2 M+2 N)}{k M(k+2 M)} b_{1} \\
& \times\left[-\sqrt{\frac{M+N}{M N}} \frac{3(k+N)}{k} J^{u(1)} J^{(\bar{\sigma} j)}\right. \\
& +\frac{3(k+N)}{(k+M)} t_{l \bar{j}}^{a} \delta^{j \bar{j}} J^{a} J^{(\bar{\sigma} l)}-3 t_{\rho \bar{\tau}}^{\alpha} \delta^{\rho \bar{\rho}} J^{\alpha} J^{(\bar{\tau} j)} \\
& \left.-\frac{\left(k^{3}+2 k^{2} M+2 k^{2} N+3 k M N+2 k+M+N\right)}{k(k+M)} \partial J^{(\bar{\sigma} j)}\right](w) \\
& +\frac{1}{(z-w)} \frac{(k+M+N)}{M} b_{1}\left[3 d^{\alpha \beta \gamma}\right. \\
& t_{\rho \bar{\tau}}^{\gamma} \delta^{\rho \bar{\sigma}} J^{\alpha} J^{\beta} J^{(\bar{\tau} j)} \\
& +\frac{3(k+N)(k+2 N)}{(k+M)(k+2 M)} d^{a b c} t_{k \bar{j}}^{c} \delta^{j \bar{j}} J^{a} J^{b} J^{(\bar{\sigma} k)} \\
& +\frac{6(k+N)(k+2 N)(M+N)}{k^{2} M N} J^{u(1)} J^{u(1)} J^{(\bar{\sigma} j)} \\
& +\frac{6(k+N)}{k N} J^{\alpha} J^{\alpha} J^{(\bar{\sigma} j)}+\sqrt{\frac{M+N}{M N}} \frac{12(k+N)}{k} \\
& t_{\sigma \bar{\tau}}^{\alpha} \delta^{\sigma \bar{\sigma}} J^{\alpha} J^{u(1)} J^{(\bar{\tau} j)} \\
& +\frac{6(k+N)(k+2 N)}{k M(k+2 M)} J^{a} J^{a} J^{(\bar{\sigma} j)} \\
& -\sqrt{\frac{M+N}{M N}} \frac{12(k+N)(k+2 N)}{k(k+2 M)} t_{k \bar{j}}^{a} \delta^{j \bar{j}} J^{a} J^{u(1)} J^{(\bar{\sigma} k)} \\
& -\frac{12(k+N)}{(k+2 M)} \delta_{\sigma \bar{v}} \delta_{k \bar{j}} J^{(\sigma \bar{j})} J^{(\bar{v} j)} J^{(\bar{\sigma} k)} \\
& -\frac{24(k+N)}{k(k+2 M)} \delta_{\sigma \bar{\tau}} \delta_{k \bar{j}} J^{(\sigma \bar{j})} J^{(\bar{\sigma} j)} J^{(\bar{\tau} k)} \\
& +\sqrt{\frac{M+N}{M N}} \frac{12(k+N)}{k} t_{\sigma \bar{\tau}}^{\alpha} \delta^{\sigma \bar{\sigma}} J^{\alpha} J^{u(1)} J^{(\bar{\tau} j)} \\
& -\frac{12(k+N)}{(k+2 M)} t_{k \bar{j}}^{a} \delta^{j \bar{j}} t_{\sigma \bar{\tau}}^{\alpha} \delta^{\sigma \bar{\sigma}} J^{\alpha} J^{a} J^{(\bar{\tau} k)} \\
& +\frac{3\left(k^{2}+2 k N+4\right)}{k} t_{\rho \bar{\tau}}^{\alpha} \delta^{\rho \bar{\sigma}} J^{\alpha} \partial J^{(\bar{\tau} j)} \\
& -\frac{3\left(k^{2}+2 k M+4\right)(k+N)(k+2 N)}{k(k+M)(k+2 M)} t_{k \bar{j}}^{a} \delta^{j \bar{j}} J^{a} \partial J^{(\bar{\sigma} k)}+\sqrt{\frac{M+N}{M N}} \\
& \times \frac{3(k+N)\left(k^{3}+2 k^{2} M+2 k^{2} N+8 k M N+4 k+8 M+8 N\right)}{k^{2}(k+2 M)} \\
& \times J^{u(1)} \partial J^{(\bar{\sigma} j)}-\frac{3\left(k^{2}-2 k M-8\right)(k+N)}{k(k+2 M)} t_{\rho \bar{\tau}}^{\alpha} \delta^{\rho \bar{\sigma}} \partial J^{\alpha} J^{(\bar{\tau} j)} \\
& -\frac{3(k+N)\left(-k^{2}+2 k N+8\right)}{k(k+2 M)} t_{k \bar{j}}^{a} \delta^{j \bar{j}} \partial J^{a} J^{(\bar{\sigma} k)}-\sqrt{\frac{M+N}{M N}} \\
& \frac{3(k+N)(k+M+N)\left(k^{2}-2 k M-2 k N-4 M N-8\right)}{k(k+2 M)} \partial J^{u(1)} \\
& \times J^{(\bar{\sigma} j)}+\frac{1}{2 k^{2}(k+M)(k+2 M)}\left(k^{5} M+k^{5} N+2 k^{4} M^{2}\right. \\
& +10 k^{4} M N+2 k^{4} N^{2}+6 k^{4}+12 k^{3} M^{2} N \\
& +12 k^{3} M N^{2}+25 k^{3} M+25 k^{3} N+12 k^{2} M^{2} N^{2}+14 k^{2} M^{2} \\
& +64 k^{2} M N+14 k^{2} N^{2}+24 k^{2} \\
& +24 k M^{2} N+24 k M N^{2}+28 k M \\
& \left.\left.+28 k N+8 M^{2}+16 M N+8 N^{2}\right) \partial^{2} J^{(\bar{\sigma} j)}\right](w)+\cdots .
\end{aligned}
$$


C.3 The OPE between $J^{(\rho \bar{i})}(z) P^{a}(w)$

The OPE between the spin- 1 current and the other spin- 3 current is summarized by

$$
\begin{aligned}
J^{(\rho \bar{i})}(z) P^{a}(w) & \frac{1}{(z-w)^{3}} \frac{\left(k^{2}-1\right)\left(k^{2}-4\right)(2 k+M+N)(3 k+2 M+2 N)}{2 k^{2}(k+M)(3 k+2 M)} \\
& \times a_{1} t_{i \bar{k}}^{a} \delta^{i \bar{i}} J^{(\rho \bar{k})}(w) \\
& +\frac{1}{(z-w)^{2}} \frac{\left(k^{2}-4\right)(3 k+2 M+2 N)}{2 k(k+M)} a_{1} \\
& \times\left[\sqrt{\frac{M+N}{M N} \frac{(k+N)}{k} t_{i \bar{k}}^{a} \delta^{i \bar{i}} J^{u(1)} J^{(\rho \bar{k})}}\right. \\
& -\frac{\left(3 k^{2}+3 k M+3 k N+M^{2}+M N\right)}{k M(3 k+2 M)} J^{a} J^{(\rho \bar{i})} \\
& +t_{\sigma \bar{\rho}}^{\alpha} \delta^{\rho \bar{\rho}} t_{i \bar{k}}^{a} \delta^{i \bar{i}} J^{\alpha} J^{(\sigma \bar{k})} \\
& +\frac{(-k-M+N)}{2(3 k+2 M)} i f^{b a c} t_{i \bar{k}}^{c} \delta^{i \bar{i}} J^{b} J^{(\rho \bar{k})} \\
& -\frac{(3 k+M+3 N)}{2(3 k+2 M)} d^{a b c} t_{i \bar{k}}^{c} \delta^{i \bar{i}} J^{b} J^{(\rho \bar{k})} \\
& \left.-\frac{\left(k^{3}+k^{2} M+2 k^{2} N+2 k M N+2 k+M+N\right)}{k(3 k+2 M)} t_{i \bar{k}}^{a} \delta^{i \bar{i}} \partial J^{(\rho \bar{k})}\right] \\
& \times(w)+\left.\frac{1}{(z-w)} J^{(\rho \bar{i})}(z) P^{a}(w)\right|_{\frac{1}{(z-w)}}+\cdots
\end{aligned}
$$

The first order pole above is given by

$$
\begin{aligned}
& \left.J^{(\rho \bar{i})}(z) P^{a}(w)\right|_{\frac{1}{(z-w)}}=-a_{1} t_{k \bar{j}}^{a} \delta_{\nu \bar{\tau}} J^{(\rho \bar{j})} J^{(\nu \bar{i})} J^{(\bar{\tau} k)} \\
& +\frac{a_{1}}{N} t_{k \bar{j}}^{a} \delta_{\sigma \bar{\tau}} J^{(\sigma \bar{j})} J^{(\rho \bar{i})} J^{(\bar{\tau} k)} \\
& +\left(\sqrt{\frac{M+N}{M N}} a_{1}+2 a_{7}\right) t_{\sigma \bar{\rho}}^{\alpha} \delta^{\rho \bar{\rho}} t_{i \bar{k}}^{a} \delta^{i \bar{i}} J^{\alpha} J^{u(1)} J^{(\sigma \bar{k})} \\
& +\left(\frac{a_{1}}{N}+a_{2}\right) t_{i \bar{k}}^{a} \delta^{i \bar{i}} J^{\alpha} J^{\alpha} J^{(\rho \bar{k})} \\
& +\frac{a_{1}}{2}(i f+d)^{\alpha \beta \gamma} t_{\sigma \bar{\rho}}^{\gamma} \delta^{\rho \bar{\rho}} t_{i \bar{k}}^{a} \delta^{i \bar{i}} J^{\alpha} J^{\beta} J^{(\sigma \bar{k})} \\
& +\left(-\frac{a_{1}}{M}-2 a_{2}+2 a_{8}\right) t_{\sigma \bar{\rho}}^{\alpha} \delta^{\rho \bar{\rho}} J^{\alpha} J^{a} J^{(\sigma \bar{i})} \\
& -\frac{a_{1}}{2}(i f+d)^{b a c} t_{\sigma \bar{\rho}}^{\alpha} \delta^{\rho \bar{\rho}} t_{i \bar{k}}^{c} \delta^{i \bar{i}} J^{\alpha} J^{b} J^{(\sigma \bar{k})} \\
& +\left(a_{3}+\frac{6}{M} a_{17}\right) t_{i \bar{k}}^{a} \delta^{i \bar{i}} J^{b} J^{b} J^{(\rho \bar{k})} \\
& +\left(2 a_{3}-2 a_{8}+\frac{6}{M} a_{17}\right) t_{i \bar{k}}^{b} \delta^{i \bar{i}} J^{a} J^{b} J^{(\rho \bar{k})} \\
& +\left(a_{4}+2 \sqrt{\frac{M+N}{M N}} a_{7}\right) t_{i \bar{k}}^{a} \delta^{i \bar{i}} J^{u(1)} J^{u(1)} J^{(\rho \bar{k})} \\
& +\left(-2 \sqrt{\frac{M+N}{M N}} a_{4}-\frac{2}{M} a_{7}\right. \\
& \left.+2 \sqrt{\frac{M+N}{M N}} a_{8}\right) J^{a} J^{u(1)} J^{(\rho \bar{i})}
\end{aligned}
$$$$
+a_{5} d^{a b c} t_{i \bar{k}}^{c} \delta^{i \bar{i}} t_{k \bar{j}}^{b} \delta_{\sigma \bar{\sigma}} J^{(\rho \bar{k})} J^{(\sigma \bar{j})} J^{(\bar{\sigma} k)}
$$$$
+a_{5} d^{a b c} t_{i \bar{k}}^{c} \delta^{i \bar{i}} t_{k \bar{j}}^{b} \delta_{\sigma \bar{\sigma}} J^{(\rho \bar{k})} J^{(\bar{\sigma} k)} J^{(\sigma \bar{j})}
$$$$
-\sqrt{\frac{M+N}{M N}} a_{7} t_{k \bar{j}}^{a} \delta_{\sigma \bar{\tau}} J^{(\rho \bar{i})} J^{(\sigma \bar{j})} J^{(\bar{\tau} k)}
$$$$
-\sqrt{\frac{M+N}{M N}} a_{7} t_{k \bar{j}}^{a} \delta_{\sigma \bar{\tau}} J^{(\rho \bar{i})} J^{(\bar{\tau} k)} J^{(\sigma \bar{j})}
$$$$
+\left(2 \sqrt{\frac{M+N}{M N}} a_{5}-a_{7}+2 a_{9}\right) d^{a b c} t_{i \bar{k}}^{b} \delta^{i \bar{i}} J^{c} J^{u(1)} J^{(\rho \bar{k})}
$$$$
+2 a_{5} d^{a b c} t_{\sigma \bar{\rho}}^{\alpha} \delta^{\rho \bar{\rho}} t_{i \bar{k}}^{b} \delta^{i \bar{i}} J^{\alpha} J^{c} J^{(\sigma \bar{k})}
$$$$
+\left(-\frac{2}{M} a_{5}-\sqrt{\frac{M+N}{M N}} a_{9}\right) d^{a b c} J^{c} J^{b}{ }^{(\rho \bar{i})}
$$$$
-a_{5}(i f+d)^{e b d} d^{a b c} t_{i \bar{k}}^{d} \delta^{i \bar{i}} J^{c} J^{e} J^{(\rho \bar{k})}
$$$$
+a_{7} i f^{a b c} t_{i \bar{k}}^{c} \delta^{i \bar{i}} J^{b} J^{u(1)} J^{(\rho \bar{k})}
$$$$
+a_{8} t_{i \bar{l}}^{a} \delta^{i \bar{i}} \delta_{\sigma \bar{\tau}} \delta_{k \bar{j}} J^{(\rho \bar{l})} J^{(\sigma \bar{j})} J^{(\bar{\tau} k)}
$$$$
+a_{8} t_{i \bar{l}}^{a} \delta^{i \bar{i}} \delta_{\sigma \bar{\tau}} \delta_{k \bar{j}} J^{(\rho \bar{l})} J^{(\bar{\tau} k)} J^{(\sigma \bar{j})}
$$$$
+\frac{3}{2} a_{17}(i f+d)^{a b e} d^{e c d} t_{i \bar{k}}^{b} \delta^{i \bar{i}} J^{c} J^{d} J^{(\rho \bar{k})}
$$$$
+\frac{3}{2} a_{17}(i f+d)^{a d e} d^{e b c} t_{i \bar{k}}^{b} \delta^{i \bar{i}} J^{c} J^{d} J^{(\rho \bar{k})}
$$$$
+\frac{3}{2} a_{17}(i f(w)+(w) d)^{a c e} d^{e b d} t_{i \bar{k}}^{b} \delta^{i \bar{i}} J^{c} J^{d} J^{(\rho \bar{k})}
$$$$
+\frac{6}{M} a_{17} t_{i \bar{k}}^{b} \delta^{i \bar{i}} J^{b} J^{a} J^{(\rho \bar{k})}
$$$$
+\left(\frac{1}{2}\left(N-\frac{1}{N}\right) a_{2}+\frac{1}{2}\left(M-\frac{1}{M}\right) a_{3}+\frac{1}{2} \frac{M+N}{M N} a_{4}\right.
$$$$
-\sqrt{\frac{M+N}{M N}} \frac{\left(M^{2}-4\right)}{2 M} a_{9}-\frac{M}{2} a_{11}-\frac{N}{2} a_{12}+a_{16}
$$$$
\left.+\frac{18-6 M^{2}+M^{4}}{2 M^{2}} a_{17}\right)
$$$$
\times t_{i \bar{k}}^{a} \delta^{i \bar{i}} \partial^{2} J^{(\rho \bar{k})}+\left(-2 a_{2}+a_{12}\right) t_{\sigma \bar{\rho}}^{\alpha}
$$$$
\times \delta^{\rho \bar{\rho}} t_{i \bar{k}}^{a} \delta^{i \bar{i}} J^{\alpha} \partial J^{(\sigma \bar{k})}+\left(\left(N-\frac{1}{N}\right) a_{2}+\frac{(M+N)}{M N} a_{4}\right.
$$$$
-(M+N) a_{8}-\frac{1}{M} a_{12}+\frac{6\left(2 M^{2}-3\right)}{M^{2}} a_{17}
$$$$
\left.+\left(M+\frac{1}{M}\right) a_{3}\right) J^{a} \partial J^{(\rho \bar{i})}
$$$$
+\left(a_{3}-2 a_{3}-a_{11}+\frac{1}{2} a_{12}\right) i f^{a b c} t_{i \bar{k}}^{c}
$$$$
\delta^{i \bar{i}} J^{b} \partial J^{(\rho \bar{k})}
$$$$
+\left(a_{3}-N a_{5}-2 \sqrt{\frac{M+N}{M N}} a_{9}-\frac{1}{2} a_{12}\right.
$$$$
\left.+\frac{3\left(M^{2}-6\right)}{M} a_{17}\right) d^{a b c} t_{i \bar{k}}^{c} \delta^{i \bar{i}} J^{b} \partial J^{(\rho \bar{k})}
$$$$
+\left(-2 a_{3}-a_{11}-\frac{1}{2} a_{13}\right) i
$$$$
f^{a b c} t_{i \bar{k}}^{b} \delta^{i \bar{i}} \partial J^{c} J^{(\rho \bar{k})}
$$ 


$$
\begin{aligned}
& +\left(-2 \sqrt{\frac{M+N}{M N}} a_{4}-N a_{7}+\frac{\left(M^{2}-4\right)}{M} a_{9}\right. \\
& \left.+\sqrt{\frac{M+N}{M N}} a_{12}\right) t_{i \bar{k}}^{a} \delta^{i \bar{i}} J^{u(1)} \partial J^{(\rho \bar{k})} \\
& +\sqrt{\frac{M+N}{M N}} a_{13} t_{i \bar{k}}^{a} \delta^{i \bar{i}} \partial J^{u(1)} J^{(\rho \bar{k})} \\
& +a_{13} t_{\sigma \bar{\rho}}^{\alpha} \delta^{\rho \bar{\rho}} t_{i \bar{k}}^{a} \delta^{i \bar{i}} \partial J^{\alpha} J^{(\sigma \bar{k})} \\
& -\frac{1}{M} a_{13} \partial J^{a} J^{(\rho \bar{i})} \\
& -\frac{1}{2} a_{13} d^{a b c} t_{i \bar{k}}^{b} \delta^{i \bar{i}} \partial J^{c} J^{(\rho \bar{k})},
\end{aligned}
$$

where we do not substitute the coefficients appearing in (5.2) and then we can observe each contribution by looking at each coefficient term.

\section{C.4 The OPE between $J^{(\bar{\sigma} j)}(z) P^{a}(w)$}

The OPE between the other spin-1 current and the other spin3 current is summarized by

$$
\begin{aligned}
& J^{(\bar{\sigma} j)}(z) P^{a}(w)=-\frac{1}{(z-w)^{3}} \\
& \times \frac{\left(k^{2}-1\right)\left(k^{2}-4\right)(2 k+M+N)(3 k+2 M+2 N)}{2 k^{2}(k+M)(3 k+2 M)} \\
& a_{1} t_{k \bar{j}}^{a} \delta^{j \bar{j}} J^{(\bar{\sigma} k)}(w) \\
&+\frac{1}{(z-w)^{2}} \frac{\left(k^{2}-4\right)(3 k+2 M+2 N)}{2 k(k+M)} a_{1} \\
& \times\left[\sqrt{\frac{M+N}{M N} \frac{(k+N)}{k} t_{k \bar{j}}^{a} \delta^{j \bar{j}} J^{u(1)} J^{(\bar{\sigma} k)}}\right. \\
&-\frac{\left(3 k^{2}+3 k M+3 k N+M^{2}+M N\right)}{k M(3 k+2 M)} J^{a} J^{(\bar{\sigma} j)}+t_{\sigma \bar{\tau}}^{\alpha} \delta^{\sigma \bar{\sigma}} \\
& \times t_{l \bar{k}}^{a} \delta^{j \bar{k}} J^{\alpha} J^{(\bar{\tau} l)} \\
&+\frac{(-k-M+N)}{2(3 k+2 M)} i f^{a b c} t_{k \bar{l}}^{c} \delta^{j \bar{l}} J^{b} J^{(\bar{\sigma} k)} \\
&-\frac{(3 k+M+3 N)}{2(3 k+2 M)} d^{a b c} t_{k \bar{l}}^{c} \delta^{j \bar{l}} J^{b} J^{(\bar{\sigma} k)} \\
&+ \frac{\left(k^{3}+k^{2} M+2 k^{2} N+2 k M N+2 k+M+N\right)}{k(3 k+2 M)} \\
& t_{k \bar{l}}^{a} \delta^{j \bar{l}} \partial J^{(\bar{\sigma} k)}(w) \\
&+ \frac{1}{(z-w)} J^{(\bar{\sigma} j)}(z) P^{a}(w) \mid \\
& \overline{(z-w)} \\
&
\end{aligned}
$$

The first order pole can be summarized by

$$
\begin{aligned}
& \left.J^{(\bar{\sigma} j)}(z) P^{a}(w)\right|_{\frac{1}{(z-w)}}=\left(t_{l \bar{k}}^{a} \delta_{\sigma \bar{v}} J^{(\bar{v} j)} J^{(\sigma \bar{k})} J^{(\bar{\rho} l)}\right. \\
& -\frac{1}{N} t_{l \bar{k}}^{a} \delta_{\sigma \bar{\tau}} J^{(\bar{\rho} j)} J^{(\sigma \bar{k})} J^{(\bar{\tau} l)}
\end{aligned}
$$

$$
\begin{aligned}
& -\sqrt{\frac{M+N}{M N}} t_{l \bar{k}}^{a} t_{\sigma \bar{\tau}}^{\alpha} \delta^{\sigma \bar{\rho}} \delta^{j \bar{k}} J^{\alpha} J^{u(1)} J^{(\bar{\tau} l)} \\
& -\frac{1}{N} t_{l \bar{k}}^{a} \delta^{j \bar{k}} J^{\alpha} J^{\alpha} J^{(\bar{\rho} l)} \\
& -\frac{1}{2}(i f+d)^{\beta \alpha \gamma} t_{l \bar{k}}^{a} t_{\nu \bar{\tau}}^{\gamma} \delta^{\nu \bar{\rho}} \delta^{j \bar{k}} J^{\alpha} J^{\beta} J^{(\bar{\tau} l)} \\
& +\frac{1}{M} t_{\sigma \bar{\tau}}^{\alpha} \delta^{\sigma \bar{\rho}} J^{\alpha} J^{a} J^{(\bar{\tau} j)} \\
& \left.+\frac{1}{2}(i f+d)^{a b c} t_{l i}^{c} t_{\sigma \bar{\tau}}^{\alpha} \delta^{\sigma \bar{\rho}} \delta^{j \bar{i}} J^{\alpha} J^{b} J^{(\bar{\tau} l)}\right) a_{1} \\
& +\left(-t_{k \bar{j}}^{a} \delta^{j \bar{j}} J^{(\bar{\rho} k)} J^{\alpha} J^{\alpha}+t_{\rho \bar{\sigma}}^{\alpha} \delta^{\rho \bar{\rho}} J^{a} J^{(\bar{\sigma} j)} J^{\alpha}\right. \\
& \left.+t_{\rho \bar{\sigma}}^{\alpha} \delta^{\rho \bar{\rho}} J^{a} J^{\alpha} J^{(\bar{\sigma} j)}\right) a_{2} \\
& +\left(-t_{k \bar{j}}^{a} \delta^{j \bar{j}} J^{(\bar{\rho} k)} J^{b} J^{b}-t_{k \bar{j}}^{b} \delta^{j \bar{j}} J^{a} J^{(\bar{\rho} k)} J^{b}\right. \\
& -t_{k \bar{j}}^{b} \delta^{j \bar{j}} J^{a} J^{b} J^{(\bar{\rho} k)} \\
& +M t_{k \bar{j}}^{a} \delta^{j \bar{j}} \partial^{2} J^{(\bar{\rho} k)}-2 i f^{b a c} t_{k \bar{j}}^{b} \delta^{j \bar{j}} J^{(\bar{\rho} k)} \partial J^{c} \\
& \left.-2 i f^{b a c} t_{k \bar{j}}^{c} \delta^{j \bar{j}} J^{b} \partial J^{(\bar{\rho} k)}\right) a_{3} \\
& \left(-t_{k \bar{j}}^{a} \delta^{j \bar{j}} J^{(\bar{\rho} k)} J^{u(1)} J^{u(1)}\right. \\
& \left.+\sqrt{\frac{M+N}{M N}} J^{a} J^{(\bar{\rho} j)} J^{u(1)}+\sqrt{\frac{M+N}{M N}} J^{a} J^{u(1)} J^{(\bar{\rho} j)}\right) a_{4} \\
& +\left(-d^{a b c} t_{l \bar{l}}^{c} \delta^{j \bar{l}} t_{k \bar{j}}^{b} \delta_{\sigma \bar{\sigma}} J^{(\bar{\rho} l)} J^{(\sigma \bar{j})} J^{(\bar{\sigma} k)}\right. \\
& -d^{a b c} t_{l \bar{l}}^{c} \delta^{j \bar{l}} t_{k \bar{j}}^{b} \delta_{\sigma \bar{\sigma}} J^{(\bar{\rho} l)} J^{(\bar{\sigma} k)} J^{(\sigma \bar{j})} \\
& -2 \sqrt{\frac{M+N}{M N}} d^{a b c} t_{k \bar{l}}^{b} \delta^{j \bar{l}} J^{c} J^{(\bar{\rho} k)} J^{u(1)} \\
& -2 d^{a b c} t_{\rho \bar{\sigma}}^{\alpha} \delta^{\rho \bar{\rho}} t_{k \bar{l}}^{b} \delta^{j \bar{l}} J^{c} J^{(\bar{\sigma} k)} J^{\alpha} \\
& +\frac{2}{M} d^{a b c} J^{c} J^{(\bar{\rho} j)} J^{b}+(i f+d)^{b e d} d^{a b c} t_{k \bar{j}}^{d} \delta^{j \bar{j}} J^{c} J^{(\bar{\rho} k)} J^{e} \\
& \left.+N d^{a b c} t_{k \bar{j}}^{b} \delta^{j \bar{j}} J^{c} \partial J^{(\bar{\rho} k)}\right) a_{5} \\
& +\left(\sqrt{\frac{M+N}{M N}} t_{l \bar{k}}^{a} \delta_{\sigma \bar{\tau}} J^{(\bar{\rho} j)} J^{(\sigma \bar{k})} J^{(\bar{\tau} l)}\right. \\
& +\sqrt{\frac{M+N}{M N}} t_{l \bar{k}}^{a} \delta_{\sigma \bar{\tau}} J^{(\bar{\rho} j)} J^{(\bar{\tau} l)} J^{(\sigma \bar{k})} \\
& -2 \sqrt{\frac{M+N}{M N}} t_{k \bar{l}}^{a} \delta^{j \bar{l}} J^{u(1)} J^{(\bar{\rho} k)} J^{u(1)} \\
& -2 t_{\rho \bar{\sigma}}^{\alpha} \delta^{\rho \bar{\rho}} \delta^{j \bar{l}} t_{k \bar{l}}^{a} J^{u(1)} J^{(\bar{\sigma} k)} J^{\alpha} \\
& +\frac{2}{M} J^{u(1)} J^{(\bar{\rho} j)} J^{a} \\
& +(i f+d)^{a b c} t_{k \bar{j}}^{c} \delta^{j \bar{j}} J^{u(1)} J^{(\bar{\rho} k)} J^{b} \\
& \left.+N t_{k \bar{j}}^{a} \delta^{j \bar{j}} J^{u(1)} \partial J^{(\bar{\rho} k)}\right) a_{7} \\
& +\left(t_{m \bar{j}}^{a} \delta^{j \bar{j}} \delta_{\sigma \bar{\tau}} \delta_{k \bar{j}} J^{(\bar{\rho} m)} J^{(\sigma \bar{j})} J^{(\bar{\tau} k)}\right. \\
& +t_{m \bar{j}}^{a} \delta^{j \bar{j}} \delta_{\sigma \bar{\tau}} \delta_{k \bar{j}} J^{(\bar{\rho} m)} J^{(\bar{\tau} k)} J^{(\sigma \bar{j})}
\end{aligned}
$$




$$
\begin{aligned}
& -2 \sqrt{\frac{M+N}{M N}} J^{a} J^{(\bar{\rho} j)} J^{u(1)}-2 t_{\rho \bar{\tau}}^{\alpha} \delta^{\rho \bar{\rho}} J^{a} J^{(\bar{\tau} j)} J^{\alpha} \\
& +2 t_{k \bar{j}}^{b} \delta^{j \bar{j}} J^{a} J^{(\bar{\rho} k)} J^{b} \\
& \left.+(M+N) J^{a} \partial J^{(\bar{\rho} j)}\right) a_{8} \\
& +\left(\sqrt{\frac{M+N}{M N}} d^{a b c} \delta^{\tau \bar{\rho}} \delta^{j \bar{k}} J^{(\bar{\tau} k)} J^{b} J^{c}\right. \\
& -d^{a b c} t_{k \bar{j}}^{b} \delta^{j \bar{j}} J^{u(1)} J^{(\bar{\rho} k)} J^{c} \\
& \left.-d^{a b c} t_{k \bar{j}}^{c} \delta^{j \bar{j}} J^{u(1)} J^{b} J^{(\bar{\rho} k)}\right) a_{9} \\
& +\left(-i f^{a b c} t_{k \bar{j}}^{b} \delta^{j \bar{j}} \partial J^{(\bar{\rho} k)} J^{c}\right. \\
& \left.-i f^{a b c} t_{k \bar{j}}^{c} \delta^{j \bar{j}} \partial J^{b} J^{(\bar{\rho} k)}\right) a_{11} \\
& +\left(-\sqrt{\frac{M+N}{M N}} t_{l \bar{k}}^{a} \delta^{j \bar{k}} J^{(\bar{\rho} l)} \partial J^{u(1)}\right. \\
& -t_{\rho \bar{\tau}}^{\alpha} \delta^{\rho \bar{\rho}} \delta^{j \bar{k}} t_{l \bar{k}}^{a} J^{(\bar{\tau} l)} \partial J^{\alpha} \\
& +\frac{1}{M} J^{(\bar{\rho} j)} \partial J^{a} \\
& +\frac{1}{2}(i f+d)^{a b c} t_{l \bar{j}}^{c} \delta^{j \bar{j}} J^{(\bar{\rho} l)} \partial J^{b} \\
& \left.+\frac{N}{2} t_{k \bar{j}}^{a} \delta^{j \bar{j}} \partial^{2} J^{(\bar{\rho} k)}\right) a_{12} \\
& +\left(-\sqrt{\frac{M+N}{M N}} t_{k \bar{l}}^{a} \delta^{j \bar{l}} \partial J^{(\bar{\rho} k)} J^{u(1)}\right. \\
& -t_{\rho \bar{\sigma}}^{\alpha} \delta^{\rho \bar{\rho}} \delta^{j \bar{l}} t_{k \bar{l}}^{a} \partial J^{(\bar{\rho} k)} J^{\alpha} \\
& +\frac{1}{M} \partial J^{(\bar{\rho} j)} J^{a} \\
& \left.+\frac{1}{2}(i f+d)^{a b c} t_{k \bar{j}}^{c} \delta^{j \bar{j}} \partial J^{(\bar{\rho} k)} J^{b}\right) a_{13} \\
& -t_{k \bar{j}}^{a} \delta^{j \bar{j}} \partial^{2} J^{(\bar{\rho} k)} a_{16} \\
& +\left(-\frac{2}{M} t_{k \bar{j}}^{a} \delta^{j \bar{j}} J^{(\bar{\rho} k)} J^{b} J^{b}\right. \\
& -\frac{1}{2}(i f+d)^{a b e} d^{e c d} t_{k \bar{j}}^{b} \delta^{j \bar{j}} J^{(\bar{\rho} k)} J^{c} J^{d} \\
& -\frac{2}{M} t_{k \bar{j}}^{b} \delta^{j \bar{j}} J^{(\bar{\rho} k)} J^{b} J^{a} \\
& -\frac{1}{2}(i f+d)^{a d e} d^{e b c} t_{k \bar{j}}^{b} \delta^{j \bar{j}} J^{(\bar{\rho} k)} J^{c} J^{d} \\
& -\frac{2}{M} t_{k \bar{j}}^{b} \delta^{j \bar{j}} J^{(\bar{\rho} k)} J^{a} J^{b} \\
& -\frac{1}{2}(i f+d)^{a c e} d^{e b d} t_{k \bar{j}}^{b} \delta^{j \bar{j}} J^{(\bar{\rho} k)} J^{c} J^{d} \\
& -\frac{2}{M} t_{k \bar{j}}^{c} \delta^{j \bar{j}} J^{a} J^{(\bar{\rho} k)} J^{c} \\
& -\frac{1}{2}(i f+d)^{a b e} d^{e c d} t_{k \bar{j}}^{c} \delta^{j \bar{j}} J^{b} J^{(\bar{\rho} k)} J^{d} \\
& -\frac{2}{M} t_{k \bar{j}}^{b} \delta^{j \bar{j}} J^{b} J^{(\bar{\rho} k)} J^{a} \\
& -\frac{1}{2}(i f+d)^{a d e} d^{e b c} t_{k \bar{j}}^{c} \delta^{j \bar{j}} J^{b} J^{(\bar{\rho} k)} J^{d}
\end{aligned}
$$

$$
\begin{aligned}
& -\frac{2}{M} t_{k \bar{j}}^{a} \delta^{j \bar{j}} J^{b} J^{(\bar{\rho} k)} J^{b} \\
& -\frac{1}{2}(i f+d)^{a c e} d^{e b d} t_{k \bar{j}}^{c} \delta^{j \bar{j}} J^{b} J^{(\bar{\rho} k)} J^{d} \\
& -\frac{2}{M} t_{k \bar{j}}^{c} \delta^{j \bar{j}} J^{a} J^{c} J^{(\bar{\rho} k)} \\
& -\frac{1}{2}(i f+d)^{a b e} d^{e c d} t_{k \bar{j}}^{d} \delta^{j \bar{j}} J^{b} J^{c} J^{(\bar{\rho} k)} \\
& -\frac{2}{M} t_{k \bar{j}}^{a} \delta^{j \bar{j}} J^{b} J^{b} J^{(\bar{\rho} k)} \\
& -\frac{1}{2}(i f+d)^{a d e} d^{e b c} t_{k \bar{j}}^{d} \delta^{j \bar{j}} J^{b} J^{c} J^{(\bar{\rho} k)} \\
& -\frac{2}{M} t_{k \bar{j}}^{b} \delta^{j \bar{j}} J^{b} J^{a} J^{(\bar{\rho} k)} \\
& \left.-\frac{1}{2}(i f+d)^{a c e} d^{e b d} t_{k \bar{j}}^{d} \delta^{j \bar{j}} J^{b} J^{c} J^{(\bar{\rho} k)}\right) a_{17},
\end{aligned}
$$

where the coefficients are in (5.2).

\section{Some OPEs in terms of coset fields in Sect. 6}

We present the coset realizations for the following OPEs

$$
\begin{aligned}
G^{+, a} & (z) G^{-, b}(w)=\frac{1}{(z-w)^{3}} k N \delta^{a b} \\
& +\frac{1}{(z-w)^{2}}\left[\frac{(k+M+N)}{M} K\right. \\
& \left.+\frac{N}{2}(i f-d)^{a b c} J^{c}+\frac{k}{2}(i f+d)^{a b c} J_{f}^{c}\right](w) \\
& +\frac{1}{(z-w)}\left[\left(t^{b} t^{a}\right)_{l \bar{i}} \delta_{\rho \bar{v}} J^{(\rho \bar{i})} J^{(\bar{v} l)}\right. \\
& -k\left(t^{a} t^{b}\right)_{j \bar{k}} \delta_{\tau \bar{\sigma}} \psi^{(\tau \bar{k})} \partial \psi^{(\bar{\sigma} j)} \\
& -\sqrt{\frac{M+N}{M N}}\left(t^{a} t^{b}\right)_{j \bar{k}} \delta_{\tau \bar{\sigma}} J^{u(1)} \psi^{(\tau \bar{k})} \psi^{(\bar{\sigma} j)} \\
& -t_{\sigma \bar{\tau}}^{\alpha}\left(t^{a} t^{b}\right)_{j \bar{k}} J^{\alpha} \psi^{(\tau \bar{k})} \psi^{(\bar{\sigma} j)} \\
& \left.+\left(t^{a} t^{c} t^{b}\right)_{j \bar{k}} \delta_{\tau \bar{\sigma}} \psi^{(\tau \bar{k})} J^{c} \psi^{(\bar{\sigma} j)}\right](w)+\cdots
\end{aligned}
$$

The first order pole can be simplified and is given by (6.2).

The OPEs with the spin- 2 currents are

$$
\begin{aligned}
G^{+, a} & (z) K^{b}(w) \\
= & -\frac{1}{(z-w)^{2}} \frac{2\left(k^{2}-1\right)(2 k+M+N)}{k(2 k+M)}\left(t^{a} t^{b}\right) j \bar{i} \\
& \times \delta_{\rho \bar{\sigma}} J^{(\rho \bar{i})} \psi^{(\bar{\sigma} j)} \\
& +\frac{1}{(z-w)}\left[-\frac{2\left(k^{2}-1\right)(2 k+M+N)}{k(2 k+M)}\left(t^{a} t^{b}\right)_{j \bar{i}}\right. \\
& \times \delta_{\rho \bar{\sigma}} \partial\left(J^{(\rho \bar{i})} \psi^{(\bar{\sigma} j)}\right) \\
& +2(k+N)\left(t^{a} t^{b}\right)_{j \bar{i}} \delta_{\rho \bar{\sigma}} \partial J^{(\rho \bar{i})} \psi^{(\bar{\sigma} j)}
\end{aligned}
$$




$$
\begin{aligned}
& -\frac{2}{k M}(k+M+N) J^{b} G^{+, a} \\
& -\frac{2}{k}(k+N) \sqrt{\frac{M+N}{M N}}\left(t^{a} t^{b}\right)_{j \bar{l}} \\
& \times \delta_{\rho \bar{\sigma}} J^{u(1)} J^{(\rho \bar{l})} \psi^{(\bar{\sigma} j)} \\
& -\left(i f-\frac{2 k+M+2 N}{2 k+M}\right)^{b c d}\left(t^{a} t^{d}\right)_{j \bar{l}} \\
& \times \delta_{\rho \bar{\sigma}} J^{c} J^{(\rho \bar{l})} \psi^{(\bar{\sigma} j)}-2 t_{\sigma \bar{\sigma}}^{\alpha}\left(t^{a} t^{b}\right)_{j \bar{l}} J^{\alpha} \\
& \left.\times J^{(\sigma \bar{l})} \psi^{(\bar{\sigma} j)}\right](w)+\cdots,
\end{aligned}
$$

and

$$
\begin{aligned}
& G^{-, a}(z) K^{b}(w) \\
& =-\frac{1}{(z-w)^{2}} \frac{2\left(k^{2}-1\right)(2 k+M+N)}{k(2 k+M)}\left(t^{b} t^{a}\right)_{k \bar{i}} \\
& \times \delta_{\rho \bar{\sigma}} J^{(\bar{\sigma} k)} \psi(\rho \bar{i}) \\
& +\frac{1}{(z-w)}\left[-\frac{2\left(k^{2}-1\right)(2 k+M+N)}{k(2 k+M)}\left(t^{b} t^{a}\right)_{k \bar{i}}\right. \\
& \times \delta_{\rho \bar{\sigma}} \partial\left(J^{(\bar{\sigma} k)} \psi(\rho \bar{i})\right) \\
& +2(k+N)\left(t^{b} t^{a}\right)_{k \bar{i}} \delta_{\rho \bar{\sigma}} \partial J^{(\bar{\sigma} k)} \psi^{(\rho \bar{i})} \\
& +\frac{2}{k M}(k+M+N) J^{b} G^{-, a} \\
& +\frac{2}{k}(k+N) \sqrt{\frac{M+N}{M N}}\left(t^{b} t^{a}\right)_{k \bar{l}} \delta_{\rho \bar{\sigma}} \\
& \times J^{u(1)} J^{(\bar{\sigma} k)} \psi^{(\rho \bar{i})} \\
& -\left(i f+\frac{2 k+M+2 N}{2 k+M}\right)^{b c d}\left(t^{d} t^{a}\right)_{k \bar{i}} \\
& \times \delta_{\rho \bar{\sigma}} J^{c} J^{(\bar{\sigma} k)} \psi^{(\rho \bar{i})}+2 t_{\rho \bar{\sigma}}^{\alpha}\left(t^{b} t^{a}\right)_{k \bar{i}} \\
& \times J^{\alpha} J^{(\bar{\sigma} k)} \psi(\overline{\bar{i})}](w)+\cdots .
\end{aligned}
$$

They are simplified and are described in (6.4) and (6.7).

\section{E The OPEs between the nonsinglet spin-2 operators in Sect. 6}

In the computation of the OPE between the nonsinglet spin-2 current (3.1) and itself, we should use the following OPEs.

- The OPEs between the first term and the remaining terms

$$
\begin{aligned}
& K^{a}(z) J^{b} J_{f}^{u(1)}(w)=\frac{1}{(z-w)} i f^{a b c} J_{f}^{u(1)} K^{c}(w)+\cdots, \\
& K^{a}(z)(i f+d)^{c b d} J^{c} J_{f}^{d}(w)= \\
& -\frac{1}{(z-w)}(i+d)^{c b d} i f^{c a e} J_{f}^{d} K^{e}(w)+\cdots, \\
& K^{a}(z) d^{b c d} J^{c} J^{d}(w)=\frac{1}{(z-w)^{2}} M d^{a b c} K^{c}
\end{aligned}
$$

$$
\begin{gathered}
+\frac{1}{(z-w)}\left[i f^{a c e} d^{b c d} K^{e} J^{d}+i f^{a d e} d^{b c d} J^{c} K^{e}\right](w)+\cdots, \\
K^{a}(z) J^{b} J^{u(1)}(w)=\frac{1}{(z-w)} i f^{a b c} J^{u(1)} K^{c}(w)+\cdots .
\end{gathered}
$$

- The OPEs between the second term and the remaining terms

$$
\begin{aligned}
& t_{j \bar{i}}^{a} \delta_{\rho \bar{\sigma}} \psi^{(\rho \bar{i})} \partial \psi^{(\bar{\sigma} j)}(z) t_{k \bar{l}}^{b} \delta_{\mu \bar{\nu}} \psi^{(\mu \bar{l})} \partial \psi^{(\bar{\nu} k)} \\
& =-\frac{1}{(z-w)^{4}} N \delta^{a b} \\
& +\frac{1}{(z-w)^{2}}\left[-\frac{2}{M} \delta^{a b} \delta_{k \bar{i}} \delta_{\rho \bar{v}} \psi^{(\rho \bar{i})} \partial \psi^{(\bar{v} k)}\right. \\
& \left.-d^{a b c} t_{k \bar{i}}^{c} \delta_{\rho \bar{v}} \psi^{(\rho \bar{i})} \partial \psi^{(\bar{v} k)}\right](w) \\
& +\frac{1}{(z-w)}\left[-\frac{1}{M} \delta^{a b} \delta_{k \bar{i}} \delta_{\rho \bar{\nu}} \partial \psi^{(\rho \bar{i})} \partial \psi^{(\bar{v} k)}\right. \\
& -\frac{1}{2}(i f+d)^{b a c} t_{k \bar{i}}^{c} \delta_{\rho \bar{v}} \partial \psi^{(\rho \bar{i})} \partial \psi^{(\bar{v} k)} \\
& -\frac{1}{M} \delta^{a b} \delta_{k \bar{i}} \delta_{\rho \bar{v}} \psi^{(\rho \bar{i})} \partial^{2} \psi^{(\bar{v} k)} \\
& \left.-\frac{1}{2}(i f+d)^{a b c} t_{k \bar{i}}^{c} \delta_{\rho \bar{v}} \psi^{(\rho \bar{i})} \partial^{2} \psi^{(\bar{v} k)}\right](w)+\cdots, \\
& \times t_{j \bar{i}}^{a} \delta_{\rho \bar{\sigma}} \psi^{(\rho \bar{i})} \partial \psi^{(\bar{\sigma} j)}(z) J^{u(1)} J_{f}^{b}(w) \\
& =\frac{1}{(z-w)^{3}} N \delta^{a b} J^{u(1)}(w) \\
& +\frac{1}{(z-w)^{2}}\left[\frac{1}{M} \delta^{a b} J^{u(1)} J_{f}^{u(1)}\right. \\
& \left.-\frac{1}{2}(i f+d)^{b a c} J^{u(1)} J_{f}^{c}\right](w) \\
& +\frac{1}{(z-w)}\left[\frac{1}{M} \delta^{a b} J^{u(1)} \partial J_{f}^{u(1)}\right. \\
& -\frac{1}{2}(i f+d)^{b a c} J^{u(1)} \partial J_{f}^{c} \\
& +\frac{1}{2}(i f+d)^{a b c} t_{j \bar{k}}^{c} \delta_{\rho \bar{\sigma}} J^{u(1)} \psi^{(\rho \bar{k})} \partial \psi^{(\bar{\sigma} j)} \\
& \left.-\frac{1}{2}(i f+d)^{b a c} t_{l \bar{i}}^{c} \delta_{\rho \bar{\sigma}} J^{u(1)} \psi^{(\rho \bar{i})} \partial \psi^{(\bar{\sigma} l)}\right](w)+\cdots, \\
& \times t_{j \bar{i}}^{a} \delta_{\rho \bar{\sigma}} \psi^{(\rho \bar{i})} \partial \psi^{(\bar{\sigma} j)}(z) \\
& \times t_{\mu \bar{\nu}}^{\alpha} t_{k \bar{l}}^{b} J^{\alpha} \psi^{(\mu \bar{l})} \psi^{(\bar{v} k)}(w) \\
& =-\frac{1}{(z-w)^{2}}\left[\frac{1}{M} \delta^{b a} \delta_{k \bar{i}}\right. \\
& \left.+\frac{1}{2}(i f+d)^{b a c} t_{k \bar{i}}^{c}\right] t_{\rho \bar{v}}^{\alpha} \\
& \times J^{\alpha} \psi^{(\rho \bar{i})} \psi^{(\bar{v} k)}(w) \\
& +\frac{1}{(z-w)}\left[-\frac{1}{M} \delta^{b a} \delta_{k \bar{i}} t_{\rho \bar{v}}^{\alpha} J^{\alpha} \partial \psi^{(\rho \bar{i})} \psi^{(\bar{v} k)}\right. \\
& -\frac{1}{2}(i f+d)^{b a c} t_{k \bar{i}}^{c} t_{\rho \bar{v}}^{\alpha} J^{\alpha} \partial \psi^{(\rho \bar{i})} \psi^{(\bar{v} k)} \\
& -\frac{1}{M} \delta^{b a} \delta_{k \bar{i}} t_{\rho \bar{v}}^{\alpha} J^{\alpha} \psi^{(\rho \bar{i})} \partial \psi^{(\bar{v} k)}
\end{aligned}
$$




$$
\begin{aligned}
& \left.-\frac{1}{2}(i f+d)^{a b c} t_{k \bar{i}}^{c} t_{\rho \bar{v}}^{\alpha} J^{\alpha} \psi^{(\rho \bar{i})} \partial \psi^{(\bar{v} k)}\right](w)+\cdots, \\
& \times t_{j \bar{i}}^{a} \delta_{\rho \bar{\sigma}} \psi^{(\rho \bar{i})} \partial \psi^{(\bar{\sigma} j)}(z) J^{b} J_{f}^{u(1)}(w) \\
& =\frac{1}{(z-w)^{2}} J^{b} J_{f}^{a}(w)+\frac{1}{(z-w)} J^{b} \partial J_{f}^{a}(w)+\cdots, \\
& \times t_{j \bar{i}}^{a} \delta_{\rho \bar{\sigma}} \psi^{(\rho \bar{i})} \partial \psi^{(\bar{\sigma} j)}(z)(i f+d)^{c b d} J^{c} J_{f}^{d}(w) \\
& =\frac{1}{(z-w)^{3}} N(i f+d)^{c b a} J^{c} \\
& +\frac{1}{(z-w)^{2}}\left[\frac{1}{M} \delta^{d a} \delta_{l \bar{i}}\right. \\
& \left.+\frac{1}{2}(i f+d)^{d a e} t_{l \bar{i}}^{e}\right] \delta_{\rho \bar{\sigma}}(i f+d)^{c b d} J^{c} \psi^{(\rho \bar{i})} \psi^{(\bar{\sigma} l)} \\
& \times(w)+\frac{1}{(z-w)}\left[\frac{1}{M} \delta^{d a} \delta_{l \bar{i}}(i f+d)^{c b d} t_{l \bar{i}}^{e}\right. \\
& \times \delta_{\rho \bar{\sigma}} J^{c} \partial\left(\psi^{(\rho \bar{i})} \psi^{(\bar{\sigma} l)}\right) \\
& +\frac{1}{2}(i f+d)^{d a e} t_{l \bar{i}}^{e} \\
& \times \delta_{\rho \bar{\sigma}}(i f+d)^{c b d} J^{c} \partial\left(\psi^{(\rho \bar{i})} \psi^{(\bar{\sigma} l)}\right) \\
& +\frac{1}{2}(i f+d)^{a d e} t_{j \bar{k}}^{e} \delta_{\rho \bar{\sigma}}(i f+d)^{c b d} \\
& \times \delta_{\rho \bar{\sigma}} J^{c} \psi^{(\rho \bar{k})} \partial \psi^{(\bar{\sigma} j)} \\
& -\frac{1}{2}(i f+d)^{d a e} t_{l \bar{i}}^{e} \delta_{\rho \bar{\sigma}}(i f+d)^{c b d} \\
& \left.\times J^{c} \psi^{(\rho \bar{i})} \partial \psi^{(\bar{\sigma} l)}\right](w)+\cdots, \\
& \times t_{j \bar{i}}^{a} \delta_{\rho \bar{\sigma}} \psi^{(\rho \bar{i})} \partial \psi^{(\bar{\sigma} j)}(z) \partial J_{f}^{b}(w) \\
& =\frac{1}{(z-w)^{4}} 3 N \delta^{a b}+\frac{1}{(z-w)^{3}}\left[\frac{2}{M} \delta^{b a} J_{f}^{u(1)}\right. \\
& \left.-(i f+d)^{b a c} J_{f}^{c}\right](w) \\
& +\frac{1}{(z-w)^{2}}\left[\frac{2}{M} \delta^{b a} \partial J_{f}^{u(1)}-(i f+d)^{b a c} \partial J_{f}^{c}\right. \\
& \left.+i f^{a b c} t_{j \bar{k}}^{c} \delta_{\rho \bar{\sigma}} \psi^{(\rho \bar{k})} \partial \psi^{(\bar{\sigma} j)}\right](w) \\
& +\frac{1}{(z-w)}\left[\frac{1}{M} \delta^{b a} \partial^{2} J_{f}^{u(1)}-\frac{1}{2}(i f+d)^{b a c} \partial^{2} J_{f}^{c}\right. \\
& \left.+i f^{a b c} t_{j \bar{k}}^{c} \delta_{\rho \bar{\sigma}} \partial\left(\psi^{(\rho \bar{k})} \partial \psi^{(\bar{\sigma} j)}\right)\right](w)+\cdots \text {. }
\end{aligned}
$$

- The OPEs between the third term and the remaining terms

$$
\begin{aligned}
& J^{u(1)} J_{f}^{a}(z) J^{u(1)} J_{f}^{b}(w)=\frac{1}{(z-w)^{4}} k N \delta^{a b} \\
& +\frac{1}{(z-w)^{3}} k i f^{a b c} J_{f}^{c} \\
& +\frac{1}{(z-w)^{2}}\left[k J_{f}^{a} J_{f}^{b}+N \delta^{a b} J^{u(1)} J^{u(1)}\right](w) \\
& +\frac{1}{(z-w)}\left[k \partial J_{f}^{a} J_{f}^{b}+N \delta^{a b} J^{u(1)} \partial J^{u(1)}\right. \\
& \left.+i f^{a b c} J^{u(1)} J^{u(1)} J_{f}^{c}\right](w)+\cdots,
\end{aligned}
$$

$$
\begin{aligned}
& J^{u(1)} J_{f}^{a}(z) t_{\mu \bar{\nu}}^{\alpha} t_{k \bar{l}}^{b} J^{\alpha} \psi^{(\mu \bar{l})} \\
& \psi^{(\bar{v} k)}(w)=\frac{1}{(z-w)} \\
& \times\left[-\frac{1}{M} \delta^{a b} \delta_{j \bar{k}} t_{\rho \bar{\sigma}}^{\alpha} J^{\alpha}\left(\left(J^{u(1)} \psi^{(\rho \bar{k})}\right) \psi^{(\bar{\sigma} j)}\right)\right. \\
& -\frac{1}{2}(i f+d)^{b a c} t_{j \bar{k}}^{c} t_{\rho \bar{\sigma}}^{\alpha} J^{\alpha}\left(\left(J^{u(1)} \psi^{(\rho \bar{k})}\right) \psi^{(\bar{\sigma} j)}\right) \\
& +\frac{1}{M} \delta^{a b} \delta_{l \bar{i}} t_{\rho \bar{\sigma}}^{\alpha} J^{\alpha} \psi^{(\rho \bar{i})} J^{u(1)} \psi^{(\bar{\sigma} l)} \\
& \left.+\frac{1}{2}(i f+d)^{a b c} t_{l \bar{i}}^{c} t_{\rho \bar{\sigma}}^{\alpha} J^{\alpha} \psi^{(\rho \bar{i})} J^{u(1)} \psi^{(\bar{\sigma} l)}\right](w) \\
& +\cdots \text {, } \\
& J^{u(1)} J_{f}^{a}(z)(i f+d)^{c b d} J^{c} J_{f}^{d}(w) \\
& =\frac{1}{(z-w)^{2}} N \delta^{d a}(i f+d)^{c b d} J^{c} J^{u(1)}(w) \\
& +\frac{1}{(z-w)}\left[N \delta^{d a}(i f+d)^{c b d} J^{c} \partial J^{u(1)}\right. \\
& \left.-i f^{d a e}(i f+d)^{c b d} J^{c} J^{u(1)} J_{f}^{e}\right](w)+\cdots, \\
& J^{u(1)} J_{f}^{a}(z) \partial J_{f}^{b}(w)=\frac{1}{(z-w)^{3}} 2 N \delta^{a b} J^{u(1)} \\
& +\frac{1}{(z-w)^{2}}\left[2 N \delta^{a b} \partial J^{u(1)}-i f^{a b c} J^{u(1)} J_{f}^{c}\right](w) \\
& +\frac{1}{(z-w)}\left[N \delta^{a b} \partial^{2} J^{u(1)}\right. \\
& \left.-i f^{a b c} \partial\left(J^{u(1)} J_{f}^{c}\right)\right](w)+\cdots, \\
& J^{u(1)} J_{f}^{a}(z) J^{b} J^{u(1)}(w)=\frac{1}{(z-w)^{2}} k J^{b} \\
& J_{f}^{a}(w) \frac{1}{(z-w)} k J^{b} \partial J_{f}^{a}(w)+\cdots .
\end{aligned}
$$

- The OPEs between the fourth term and the remaining terms

$$
\begin{aligned}
t_{\rho \bar{\sigma}}^{\alpha} t_{j \bar{i}}^{a} J^{\alpha} \psi^{(\rho \bar{i})} \psi^{(\bar{\sigma} j)}(z) t_{\mu \bar{\nu}}^{\beta} t_{k \bar{l}}^{b} J^{\beta} \psi^{(\mu \bar{l})} \psi^{(\bar{v} k)}(w) & \\
= & \frac{1}{(z-w)^{4}} k\left(N^{2}-1\right) \delta^{a b} \\
& -\frac{1}{(z-w)^{3}} k\left(N-\frac{1}{N}\right) i f^{a b c} J_{f}^{c}(w) \\
& +\frac{1}{(z-w)^{2}}\left[k \left(\delta_{\rho \bar{v}} \delta_{\mu \bar{\sigma}}\right.\right. \\
& \left.-\frac{1}{N} \delta_{\rho \bar{\sigma}} \delta_{\mu \bar{v}}\right) t_{j \bar{i}}^{a} t_{k \bar{l}}^{b}\left(\psi^{(\rho \bar{i})} \psi^{(\bar{\sigma} j)}\right)\left(\psi^{(\mu \bar{l})} \psi^{(\bar{v} k)}\right) \\
& -N\left(\frac{2}{M} \delta^{a b} \delta_{k \bar{i}} t_{\rho \bar{v}}^{\gamma}+d^{a b c} t_{\rho \bar{v}}^{\gamma} t_{k \bar{i}}^{c}\right) J^{\gamma} \psi^{(\rho \bar{i})} \psi^{(\bar{v} k)} \\
& \left.+\delta^{a b} J^{\alpha} J^{\alpha}\right](w) \\
& +\frac{1}{(z-w)}\left[k\left(\delta_{\rho \bar{\nu}} \delta_{\mu \bar{\sigma}}-\frac{1}{N} \delta_{\rho \bar{\sigma}} \delta_{\mu \bar{\nu}}\right)\right. \\
& \times t_{j \bar{i}}^{a} t_{k \bar{l}}^{b}\left(\partial\left(\psi^{(\rho \bar{i})} \psi^{(\bar{\sigma} j)}\right)\right)\left(\psi^{(\mu \bar{l})} \psi^{(\bar{v} k)}\right)
\end{aligned}
$$




$$
\begin{aligned}
& -i f^{\beta \alpha \gamma} t_{\rho \bar{\sigma}}^{\alpha} t_{\mu \bar{\nu}}^{\beta} t_{j \bar{i}}^{a} t_{k \bar{l}}^{b}\left(J^{\gamma} \psi^{(\rho \bar{i})} \psi^{(\bar{\sigma} j)}\right) \\
& \times\left(\psi^{(\mu \bar{l})} \psi^{(\bar{v} k)}\right)+\delta^{a b} J^{\alpha} \partial J^{\alpha}+\left(\frac{1}{N} \delta^{\alpha \beta} \delta_{\rho \bar{v}}\right. \\
& \left.+\frac{1}{2}(i f+d)^{\alpha \beta \gamma} t_{\rho \bar{v}}^{\gamma}\right)\left(\frac{1}{M} \delta^{b a} \delta_{k \bar{i}}\right. \\
& \left.+\frac{1}{2}(i f+d)^{b a c} t_{k \bar{i}}^{c}\right) J^{\beta} J^{\alpha} \psi^{(\rho \bar{i})} \psi^{(\bar{v} k)} \\
& -\left(\frac{1}{N} \delta^{\alpha \beta} \delta_{\mu \bar{\sigma}}+\frac{1}{2}(i f+d)^{\beta \alpha \gamma} t_{\mu \bar{\sigma}}^{\gamma}\right)\left(\frac{1}{M} \delta^{b a} \delta_{j \bar{l}}\right. \\
& \left.\left.+\frac{1}{2}(i f+d)^{a b c} t_{j \bar{l}}^{c}\right) J^{\beta} J^{\alpha} \psi^{(\mu \bar{l})} \psi^{(\bar{\sigma} j)}\right](w) \\
& \times+\cdots, \\
\times & t_{\rho \bar{\sigma}}^{\alpha} t_{j \bar{i}}^{a} J^{\alpha} \psi^{(\rho \bar{i})} \psi^{(\bar{\sigma} j)}(z)(i f+d)^{c b d} J^{c} J_{f}^{d}(w) \\
= & \frac{1}{(z-w)}(i f+d)^{c b d} i f^{a d e} t_{\rho \bar{\sigma}}^{\alpha} \\
& \times t_{j \bar{k}}^{e} J^{c} J^{\alpha} \psi^{(\rho \bar{k})} \psi^{(\bar{\sigma} j)}+\cdots, \\
t_{\rho \bar{\sigma}}^{\alpha} & t_{j \bar{i}}^{a} J^{\alpha} \psi^{(\rho \bar{i})} \psi^{(\bar{\sigma} j)}(z) \partial J_{f}^{b}(w) \\
= & \frac{1}{(z-w)^{2}} i f^{a b c} t_{\rho \bar{\sigma}}^{\alpha} t_{j \bar{k}}^{c} J^{\alpha} \psi^{(\rho \bar{k})} \psi^{(\bar{\sigma} j)} \\
& +\frac{1}{(z-w)} i f^{a b c} t_{\rho \bar{\sigma}}^{\alpha} t_{j \bar{k}}^{c} \partial\left(J^{\alpha} \psi^{(\rho \bar{k})} \psi^{(\bar{\sigma} j)}\right)+\cdots
\end{aligned}
$$

- The OPEs between the fifth term and the remaining terms

$$
\begin{aligned}
J^{a} & J_{f}^{u(1)}(z) J^{b} J_{f}^{u(1)}(w)=\frac{1}{(z-w)^{4}} k M N \delta^{a b} \\
& +\frac{1}{(z-w)^{3}} M N i f^{a b c} J^{c}(w) \\
& +\frac{1}{(z-w)^{2}}\left[k \delta^{a b} J_{f}^{u(1)} J_{f}^{u(1)}\right. \\
& \left.+i f^{a b c} M N \partial J^{c}+M N J^{b} J^{a}\right](w) \\
& +\frac{1}{(z-w)}\left[k \delta^{a b} \partial J_{f}^{u(1)} J_{f}^{u(1)}\right. \\
& \left.+i f^{a b c}\left(\left(J^{c} J_{f}^{u(1)}\right) J_{f}^{u(1)}\right)+M N J^{b} \partial J^{a}\right](w)+\cdots \\
J^{a} & J_{f}^{u(1)}(z)(i f+d)^{c b d} J^{c} J_{f}^{d}(w) \\
= & \frac{1}{(z-w)^{2}} k(i f+d)^{a b c} J_{f}^{c} J_{f}^{u(1)}(w) \\
& +\frac{1}{(z-w)}\left[k(i f+d)^{a b c} J_{f}^{c} \partial J_{f}^{u(1)}\right. \\
& \left.-(i f+d)^{c b d} i f^{c a e} J_{f}^{d} J^{e} J_{f}^{u(1)}\right](w) \\
& \times+\cdots \\
J^{a} & J_{f}^{u(1)}(z) d^{b c d} J^{c} J^{d}(w) \\
= & \frac{1}{(z-w)^{2}}(2 k+M) d^{a b c} J_{f}^{u(1)} J^{c}(w)
\end{aligned}
$$

$$
\begin{aligned}
& +\frac{1}{(z-w)}\left[(2 k+M) d^{a b c} \partial\left(J_{f}^{u(1)} J^{c}\right)\right. \\
& -(2 k+M) d^{a b c} J_{f}^{u(1)} \partial J^{c} \\
& \left.+i f^{a b c} d^{c d e} J_{f}^{u(1)} J^{d} J^{e}\right](w)+\cdots, \\
& J^{a} J_{f}^{u(1)}(z) J^{b} J^{u(1)}(w)=\frac{1}{(z-w)^{2}} k \delta^{a b} \\
& J^{u(1)} J_{f}^{u(1)}(w)+\frac{1}{(z-w)}\left[k \delta^{a b} J^{u(1)} \partial J_{f}^{u(1)}\right. \\
& \left.+i f^{a b c} J^{u(1)} J^{c} J_{f}^{u(1)}\right](w)+\cdots .
\end{aligned}
$$

- The OPEs between the sixth term and the remaining terms

$$
\begin{aligned}
(i f & +d)^{f a c} J^{f} J_{f}^{c}(z)(i f+d)^{d b e} J^{d} J_{f}^{e}(w) \\
= & \frac{1}{(z-w)^{4}} k N(i f+d)^{f a c}(i f+d)^{f b c} \\
& +\frac{1}{(z-w)^{3}}\left[k i f^{c e g}(i f+d)^{f a c}(i f+d)^{f b e} J_{f}^{g}\right. \\
& \left.-N i f^{d f g}(i f+d)^{f a c}(i f+d)^{d b c} J^{g}\right](w) \\
& +\frac{1}{(z-w)^{2}}\left[k(i f+d)^{f a c}(i f+d)^{f b e} J_{f}^{c} J_{f}^{e}\right. \\
& -N i f^{d f g}(i f+d)^{f a c}(i f+d)^{d b c} \partial J^{g} \\
& -f^{d h f} f^{e c g}(i f+d)^{h a c}(i f+d)^{d b e} J^{f} J_{f}^{g} \\
& \left.+N(i f+d)^{f a c}(i f+d)^{d b c} J^{d} J^{f}\right](w) \\
& +\frac{1}{(z-w)}\left[k(i f+d)^{f a c}(i f+d)^{f b e} \partial J_{f}^{c} J_{f}^{e}\right. \\
& +N(i f+d)^{f a c}(i f+d)^{d b c} J^{d} \partial J^{f} \\
& -i f^{d g f}(i f+d)^{g a c}(i f+d)^{d b e}\left(\left(J^{f} J_{f}^{c}\right) J_{f}^{e}\right) \\
& \left.-i f^{e c g}(i f+d)^{h a c}(i f+d)^{d b e} J^{d} J^{h} J_{f}^{g}\right](w) \\
& +\cdots,
\end{aligned}
$$$$
(i f+d)^{f a c} J^{f} J_{f}^{c}(z) \partial J_{f}^{b}(w)
$$$$
=\frac{1}{(z-w)^{3}} 2 N \delta^{b c}(i f+d)^{d a c} J^{d}(w)
$$$$
+\frac{1}{(z-w)^{2}}\left[2 N \delta^{b c}(i f+d)^{d a c} \partial J^{d}\right.
$$$$
\left.-i f^{b c g}(i f+d)^{d a c} J^{d} J_{f}^{g}\right](w)
$$$$
+\frac{1}{(z-w)}\left[N \delta^{b c}(i f+d)^{d a c} \partial^{2} J^{d}\right.
$$$$
\left.-i f^{b c g}(i f+d)^{d a c} \partial\left(J^{d} J_{f}^{g}\right)\right](w)+\cdots,
$$$$
(i f+d)^{f a c} J^{f} J_{f}^{c}(z) d^{b d e} J^{d} J^{e}(w)
$$$$
=\frac{1}{(z-w)^{2}}(2 k+M) d^{d b c}(i f+d)^{d a e} J^{c} J_{f}^{e}(w)
$$$$
+\frac{1}{(z-w)}\left[(2 k+M) d^{d b c}(i f+d)^{d a e} \partial\left(J^{c} J_{f}^{e}\right)\right.
$$ 


$$
\begin{aligned}
& -(2 k+M) d^{d b c}(i f+d)^{d a e} \partial J^{c} J_{f}^{e} \\
+ & \left.i f^{f b c} d^{c d e}(i f+d)^{f a g} J^{d} J^{e} J_{f}^{g}\right](w)+\cdots, \\
(i f+d)^{f a c} J^{f} J_{f}^{c}(z) J^{b} J^{u(1)}(w) & \\
= & \frac{1}{(z-w)^{2}} k(i f+d)^{b a c} J^{u(1)} J_{f}^{c} \\
& +\frac{1}{(z-w)}\left[k(i f+d)^{b a c} \partial\left(J^{u(1)} J_{f}^{c}\right)\right. \\
& -k(i f+d)^{b a c} \partial J^{u(1)} J_{f}^{c} \\
& \left.+i f^{e b d}(i f+d)^{e a c} J^{d} J^{u(1)} J_{f}^{c}\right](w)+\cdots .
\end{aligned}
$$

- The OPEs between the seventh term and the remaining terms

$$
\begin{aligned}
\partial J_{f}^{a}(z) \partial J_{f}^{b}(w)= & -\frac{1}{(z-w)^{4}} 6 N \delta^{a b} \\
& -\frac{1}{(z-w)^{3}} 2 i f^{a b c} J_{f}^{c}(w) \\
& -\frac{1}{(z-w)^{2}} i f^{a b c} \partial J_{f}^{c}(w)+\cdots
\end{aligned}
$$

- The OPEs between the eighth term and the remaining terms

$$
\begin{aligned}
& d^{a c d} J^{c} J^{d}(z) J^{b} J^{u(1)}(w)=\frac{1}{(z-w)^{2}} \\
& \times(2 k+M) d^{b a c} J^{c} J^{u(1)}(w) \\
& +\frac{1}{(z-w)}\left[(2 k+M) d^{b a c} \partial J^{c} J^{u(1)}\right. \\
& \left.-i f^{b a c} d^{c d e} J^{d} J^{e} J^{u(1)}\right](w)+\cdots
\end{aligned}
$$

- The OPE between the last term and itself

$$
\begin{aligned}
& J^{a} J^{u(1)}(z) J^{b} J^{u(1)}(w)=\frac{1}{(z-w)^{4}} k^{2} \delta^{a b} \\
& +\frac{1}{(z-w)^{3}} k i f^{a b c} J^{c}(w) \\
& +\frac{1}{(z-w)^{2}}\left[k \delta^{a b} J^{u(1)} J^{u(1)}\right. \\
& \left.+k i f^{a b c} \partial J^{c}+k J^{b} J^{a}\right](w) \\
& +\frac{1}{(z-w)}\left[k \delta^{a b} \partial J^{u(1)} J^{u(1)}\right. \\
& \left.+i f^{a b c}\left(\left(J^{c} J^{u(1)}\right) J^{u(1)}\right)+k J^{b} \partial J^{a}\right](w)+\cdots
\end{aligned}
$$

The other OPEs where the order of the operators in the left hand side is reversed can be calculated by using the above OPEs with the appropriate contributions from the higher order poles.

\section{F The OPEs between the singlet spin-2 operators in}

Sect. 7

In order to calculate the OPE in (7.1), we need to compute the following OPEs

$$
\begin{aligned}
\delta_{\rho \bar{\sigma}} & \delta_{j \bar{i}} J^{(\rho \bar{i})} J^{(\bar{\sigma} j)}(z) \delta_{\mu \bar{v}} \delta_{l \bar{k}} J^{(\mu \bar{k})} J^{(\bar{v} l)}(w) \\
= & \frac{1}{(z-w)^{4}} k N M(k-N-M) \\
& +\frac{1}{(z-w)^{2}}\left[2(k+N+M)^{2} T_{\text {boson }}\right. \\
& -2(M+k)(k+M+N) J^{a} J^{a} \\
& +\left(\frac{M}{2(k+N)}\right)^{2} 4(N+k) J^{\alpha} J^{\alpha} \\
& +\left(\frac{M+N}{2 k}\right)^{2} 4 k J^{u(1)} J^{u(1)} \\
& \left.+k(M+N) M N \sqrt{\frac{M+N}{M N}} \partial J^{u(1)}\right](w) \\
& +\mathcal{O}\left(\frac{1}{(z-w)}\right)+\cdots,
\end{aligned}
$$

$J^{\alpha} J^{\alpha}(z) J^{\beta} J^{\beta}(z)=\frac{1}{(z-w)^{4}} 2 k(N+k)\left(N^{2}-1\right)$

$$
+\frac{1}{(z-w)^{2}} 4(N+k) J^{\beta} J^{\beta}+\mathcal{O}\left(\frac{1}{(z-w)}\right)+\cdots,
$$$$
J^{\alpha} J_{f}^{\alpha}(z) J^{\beta} J_{f}^{\beta}(w)
$$$$
=\frac{1}{(z-w)^{4}} M k\left(N^{2}-1\right)
$$$$
+\frac{1}{(z-w)^{2}}\left[k J_{f}^{\alpha} J_{f}^{\alpha}\right.
$$

$$
\left.-2 N J^{\alpha} J_{f}^{\alpha}+M J^{\alpha} J^{\alpha}\right](w)
$$

$$
+\mathcal{O}\left(\frac{1}{(z-w)}\right)+\cdots,
$$

$$
J^{u(1)} J_{f}^{u(1)}(z) J^{u(1)} J_{f}^{u(1)}(w)=\frac{1}{(z-w)^{4}} k M N
$$

$$
+\frac{1}{(z-w)^{2}}\left[k J_{f}^{u(1)} J_{f}^{u(1)}+M N J^{u(1)} J^{u(1)}\right](w)
$$$$
+\mathcal{O}\left(\frac{1}{(z-w)}\right)+\cdots,
$$

$J^{a} J_{f}^{a}(z) J^{b} J_{f}^{b}(w)=\frac{1}{(z-w)^{4}} k N\left(M^{2}-1\right)$

$$
\begin{aligned}
&+\frac{1}{(z-w)^{2}}\left[k J_{f}^{a} J_{f}^{a}-2 M J^{a} J_{f}^{a}+N J^{a} J^{a}\right](w) \\
&+\mathcal{O}\left(\frac{1}{(z-w)}\right)+\cdots, \\
& \delta_{\rho \bar{\sigma}} \delta_{j \bar{i}} \partial \psi^{(\rho \bar{i})} \psi^{(\bar{\sigma} j)}(z) \delta_{\mu \bar{v}} \delta_{l \bar{k}} \partial \psi^{(\mu \bar{k})} \psi^{(\bar{v} l)}(w)
\end{aligned}
$$




$$
\begin{aligned}
&=-\frac{1}{(z-w)^{4}} N M \\
&+\frac{1}{(z-w)^{2}} 2 \delta_{\mu \bar{v}} \delta_{l \bar{k}} \partial \psi^{(\mu \bar{k})} \psi^{(\bar{v} l)}(w) \\
&+\mathcal{O}\left(\frac{1}{(z-w)}\right)+\cdots, \\
& J^{a} J^{a}(z) J^{b} J^{b}(w)=\frac{1}{(z-w)^{4}} 2 k(M+k)\left(M^{2}-1\right) \\
&+\frac{1}{(z-w)^{2}} 4(M+k) J^{a} J^{a} \\
&+\mathcal{O}\left(\frac{1}{(z-w)}\right)+\cdots, \\
& \delta_{\rho \bar{\sigma}} \delta_{j \bar{i}} \psi^{(\rho \bar{i})} \partial \psi^{(\bar{\sigma} j)}(z) \delta_{\mu \bar{\nu}} \delta_{l \bar{k}} \psi^{(\mu \bar{k})} \partial \psi^{(\bar{v} l)}(w) \\
&=-\frac{1}{(z-w)^{4}} N M \\
&-\frac{1}{(z-w)^{2}} 2 \delta_{\mu \bar{\nu}} \delta_{l \bar{k}} \psi^{(\mu \bar{k})} \partial \psi^{(\bar{v} l)}(w) \\
&+\mathcal{O}\left(\frac{1}{(z-w)}\right)+\cdots, \\
& J^{u(1)} J^{u(1)}(z) J^{u(1)} J^{u(1)}(w) \frac{1}{(z-w)^{4}} 2 k^{2}+\frac{1}{(z-w)^{2}} 4 k J^{u(1)} J^{u(1)} \\
&+\mathcal{O}\left(\frac{1}{(z-w)}\right)+\cdots, \\
&+\mathcal{O}\left(\frac{1}{(z-w)}\right)+\cdots, \\
& J^{u(1)}(z) \partial J^{u(1)}(w)=-\frac{1}{(z-w)^{4}} 6 k \\
&
\end{aligned}
$$

We only present the OPEs up to the second order pole in (F.1) where the bosonic stress energy tensor $T_{\text {boson }}$ is given by the footnote 9. Other OPEs between the operators of $W^{-(2), 0}$ can be performed similarly.

\section{G Some of the defining relations in Sect. 8}

For convenience, we present the previous relations in [72].

The OPEs between the supersymmetry generators of spin$\frac{3}{2}$ are given by

$$
\begin{aligned}
\hat{G}_{11}(z) \hat{G}_{11}(w)= & \frac{1}{(z-w)} \frac{4}{(N+k+2)}\left[-\hat{A}_{+} \hat{B}_{-}\right](w)+\cdots, \\
\hat{G}_{11}(z) \hat{G}_{12}(w)= & \frac{1}{(z-w)^{2}}\left[4 i \gamma_{A} \hat{A}_{+}\right](w) \\
& +\frac{1}{(z-w)}\left[2 i \gamma_{A} \partial \hat{A}_{+}\right. \\
& \left.+\frac{4}{(N+k+2)} \hat{A}_{+} \hat{B}_{3}\right](w)+\cdots, \\
\hat{G}_{11}(z) \hat{G}_{21}(w)= & \frac{1}{(z-w)^{2}}\left[-4 i \gamma_{B} \hat{B}_{-}\right](w)
\end{aligned}
$$

$$
\begin{aligned}
& +\frac{1}{(z-w)}\left[-2 i \gamma_{B} \partial \hat{B}_{-}\right. \\
& \left.+\frac{4}{(N+k+2)} \hat{A}_{3} \hat{B}_{-}\right](w)+\cdots, \\
& \hat{G}_{11}(z) \hat{G}_{22}(w)=\frac{1}{(z-w)^{3}} \frac{2}{3} c_{\text {Wolf }} \\
& +\frac{1}{(z-w)^{2}}\left[4 i\left(\gamma_{A} \hat{A}_{3}-\gamma_{B} \hat{B}_{3}\right)\right](w) \\
& +\frac{1}{(z-w)}\left[2 \hat{T}+2 i \partial\left(\gamma_{A} \hat{A}_{3}-\gamma_{B} \hat{B}_{3}\right)\right. \\
& +\frac{2}{(k+N+2)}\left(\hat{A}_{i} \hat{A}_{i}+\hat{B}_{i} \hat{B}_{i}\right. \\
& \left.\left.+2 \hat{A}_{3} \hat{B}_{3}\right)\right](w)+\cdots, \\
& \hat{G}_{12}(z) \hat{G}_{12}(w)=\frac{1}{(z-w)} \frac{4}{(N+k+2)}\left[\hat{A}_{+} \hat{B}_{+}\right](w)+\cdots, \\
& \hat{G}_{12}(z) \hat{G}_{21}(w)=\frac{1}{(z-w)^{3}} \frac{2}{3} c_{\text {Wolf }}+\frac{1}{(z-w)^{2}}\left[4 i \left(\gamma_{A} \hat{A}_{3}\right.\right. \\
& \left.\left.+\gamma_{B} \hat{B}_{3}\right)\right](w)+\frac{1}{(z-w)}[2 \hat{T} \\
& +2 i \partial\left(\gamma_{A} \hat{A}_{3}+\gamma_{B} \hat{B}_{3}\right)+\frac{2}{(k+N+2)}\left(\hat{A}_{i} \hat{A}_{i}\right. \\
& \left.\left.+\hat{B}_{i} \hat{B}_{i}-2 \hat{A}_{3} \hat{B}_{3}\right)\right](w)+\cdots, \\
& \hat{G}_{12}(z) \hat{G}_{22}(w)=\frac{1}{(z-w)^{2}}\left[-4 i \gamma_{B} \hat{B}_{+}\right](w) \\
& +\frac{1}{(z-w)}\left[-2 i \gamma_{B} \partial \hat{B}_{+}\right. \\
& \left.+\frac{4}{(N+k+2)} \hat{A}_{3} \hat{B}_{+}\right](w)+\cdots, \\
& \hat{G}_{21}(z) \hat{G}_{21}(w)=\frac{1}{(z-w)} \frac{4}{(N+k+2)}\left[\hat{A}_{-} \hat{B}_{-}\right](w)+\cdots, \\
& \hat{G}_{21}(z) \hat{G}_{22}(w)=\frac{1}{(z-w)^{2}}\left[4 i \gamma_{A} \hat{A}_{-}\right](w) \\
& +\frac{1}{(z-w)}\left[2 i \gamma_{A} \partial \hat{A}_{-}\right. \\
& \left.+\frac{4}{(N+k+2)} \hat{A}_{-} \hat{B}_{3}\right](w)+\cdots, \\
& \hat{G}_{22}(z) \hat{G}_{22}(w)=\frac{1}{(z-w)} \frac{4}{(N+k+2)} \\
& \times\left[-\hat{A}_{-} \hat{B}_{+}\right](w)+\cdots,
\end{aligned}
$$

where the two parameters are given by $\gamma_{A} \equiv \frac{N}{N+k+2}, \gamma_{B} \equiv$ $\frac{k}{N+k+2}$, and we introduce the spin- 1 currents $\hat{A}_{ \pm}(z) \equiv \hat{A}_{1} \pm$ $i \hat{A}_{2}(z)$ and $\hat{B}_{ \pm}(z) \equiv \hat{B}_{1} \pm i \hat{B}_{2}(z)$ and the central term above is given by $c_{\text {Wolf }}=\frac{6 k N}{(2+k+N)}$.

The higher spin- $\frac{3}{2}$ currents can be obtained from the following OPEs

$$
\begin{aligned}
& \hat{G}_{21}(z) T^{(1)}(w) \\
& \quad=\frac{1}{(z-w)}\left[\hat{G}_{21}+2 T_{+}^{\left(\frac{3}{2}\right)} \equiv \hat{G}_{21}^{\prime}\right](w)+\cdots, \\
& \hat{G}_{12}(z) T^{(1)}(w)
\end{aligned}
$$




$$
=\frac{1}{(z-w)}\left[-\hat{G}_{12}+2 T_{-}^{\left(\frac{3}{2}\right)} \equiv \hat{G}_{12}^{\prime}\right](w)+\cdots,
$$

$\hat{G}_{11}(z) T^{(1)}(w)$

$$
=\frac{1}{(z-w)}\left[\hat{G}_{11}+2 U^{\left(\frac{3}{2}\right)} \equiv \hat{G}_{11}^{\prime}\right](w)+\cdots,
$$

$\hat{G}_{22}(z) T^{(1)}(w)$

$$
=\frac{1}{(z-w)}\left[-\hat{G}_{22}+2 V^{\left(\frac{3}{2}\right)} \equiv \hat{G}_{22}^{\prime}\right](w)+\cdots .
$$

The higher spin- 2 currents are determined by

$$
\begin{aligned}
& \hat{G}_{11}(z) \hat{G}_{12}^{\prime}(w)=\frac{1}{(z-w)}\left[-2 U_{-}^{(2)}\right. \\
& \left.-\frac{4}{(N+k+2)} \hat{A}_{+} B_{3}\right](w)+\cdots, \\
& \hat{G}_{21}(z) \hat{G}_{22}^{\prime}(w)=\frac{1}{(z-w)}\left[2 V_{+}^{(2)}\right. \\
& \left.-\frac{4}{(N+k+2)} \hat{A}_{-} B_{3}\right](w)+\cdots, \\
& \hat{G}_{11}(z) \hat{G}_{21}^{\prime}(w)=\frac{1}{(z-w)}\left[-2 U_{+}^{(2)}\right. \\
& \left.-\frac{4}{(N+k+2)} \hat{A}_{3} \hat{B}_{-}\right](w)+\cdots, \\
& \hat{G}_{12}(z) \hat{G}_{22}^{\prime}(w)=\frac{1}{(z-w)}\left[2 V_{-}^{(2)}\right. \\
& \left.-\frac{4}{(N+k+2)} \hat{A}_{3} \hat{B}_{+}\right](w) \\
& \hat{G}_{12}(z) \hat{G}_{21}^{\prime}(w)=\frac{1}{(z-w)^{2}} 2 T^{(1)}(w)+\frac{1}{(z-w)} \\
& \times\left[-2 T^{(2)}+\partial T^{(1)}+\frac{2(k+N)}{(k+N+2 k N)} \hat{T}\right. \\
& +\frac{2}{(N+k+2)}\left(\hat{A}_{i} \hat{A}_{i}+\hat{B}_{i} \hat{B}_{i}\right. \\
& \left.\left.-2 \hat{A}_{3} \hat{B}_{3}\right)\right](w)+\cdots, \\
& +\frac{1}{(z-w)}\left[2 W^{(2)}+\partial T^{(1)}-2 \hat{T}\right. \\
& \left.-\frac{2}{(N+k+2)}\left(\hat{A}_{i} \hat{A}_{i}+\hat{B}_{i} \hat{B}_{i}+2 \hat{A}_{3} \hat{B}_{3}\right)\right](w)
\end{aligned}
$$$$
\hat{G}_{21}(z) \hat{G}_{12}^{\prime}(w)=\frac{1}{(z-w)^{2}} 2 T^{(1)}(w)
$$

$$
\begin{aligned}
& +\frac{1}{(z-w)}\left[2 T^{(2)}+\partial T^{(1)}-\frac{2(k+N)}{(k+N+2 k N)} \hat{T}\right. \\
& \left.-\frac{2}{(N+k+2)}\left(\hat{A}_{i} \hat{A}_{i}+\hat{B}_{i} \hat{B}_{i}-2 \hat{A}_{3} \hat{B}_{3}\right)\right](w)
\end{aligned}
$$$$
\hat{G}_{22}(z) \hat{G}_{11}^{\prime}(w)=\frac{1}{(z-w)^{2}} 2 T^{(1)}(w)
$$$$
+\frac{1}{(z-w)}\left[-2 W^{(2)}+\partial T^{(1)}+2 \hat{T}\right.
$$$$
\left.+\frac{2}{(N+k+2)}\left(\hat{A}_{i} \hat{A}_{i}+\hat{B}_{i} \hat{B}_{i}+2 \hat{A}_{3} \hat{B}_{3}\right)\right](w)
$$

$$
+\cdots .
$$

Here $\hat{G}_{m n}^{\prime}$ is defined in (G.2).

Similarly, the higher spin- $\frac{5}{2}$ currents can be obtained from

$$
\begin{aligned}
\hat{G}_{21}(z) U_{-}^{(2)}(w)= & \frac{1}{(z-w)^{2}}\left[\frac{(N+2 k)}{(N+k+2)} \hat{G}_{11}\right. \\
& \left.+\frac{2(N+2 k+1)}{(N+k+2)} U^{\left(\frac{3}{2}\right)}\right](w) \\
& +\frac{1}{(z-w)}\left[U^{\left(\frac{5}{2}\right)}+\frac{1}{3} \partial(\text { pole- } 2)\right](w)+\cdots, \\
\hat{G}_{21}(z) V_{-}^{(2)}(w)= & \frac{1}{(z-w)^{2}}\left[-\frac{(2 N+k)}{(N+k+2)} \hat{G}_{22}\right. \\
& \left.+\frac{2(2 N+k+1)}{(N+k+2)} V^{\left(\frac{3}{2}\right)}\right](w) \\
& +\frac{1}{(z-w)}\left[V^{\left(\frac{5}{2}\right)}+\frac{1}{3} \partial(\text { pole- } 2)\right](w)+\cdots, \\
\hat{G}_{21}(z) W^{(2)}(w)= & \frac{1}{(z-w)^{2}}\left[\frac{(N+2 k+1)}{(N+k+2)} \hat{G}_{21}\right. \\
& \left.+\frac{(k-N)}{(N+k+2)} T_{+}^{\left(\frac{3}{2}\right)}\right](w) \\
& +\frac{1}{(z-w)}\left[W_{+}^{\left(\frac{5}{2}\right)}+\frac{1}{3} \partial(\text { pole-2) }](w)+\cdots,\right. \\
& +\frac{1}{(z-w)}\left[\frac{(N+2 k+1)}{(N+k+2)} \hat{G}_{12}\right. \\
\hat{G}_{12}(z) W^{(2)}(w)= & \left.+\frac{(N-k)}{(N+k+2)} T_{-}^{\left(\frac{3}{2}\right)}\right](w) \\
& \left.\frac{1}{\left(\frac{5}{2}\right)}+\frac{1}{3} \partial(\text { pole- } 2)\right](w)+\cdots \\
& \\
&
\end{aligned}
$$

In next Appendix, we express the higher spin- $\frac{5}{2}$ currents for fixed $N=5$ with $M=2$.

\section{H The higher spin- $\frac{5}{2}$ currents for fixed $N=5$ and $M=2$ in Sect. 8}

We present the higher spin- $\frac{5}{2}$ currents as follows:

$$
\begin{aligned}
U^{\left(\frac{5}{2}\right)}= & \left(\frac{1}{k+7}\right)^{3 / 2}\left[i f ^ { 3 1 d } \left(\frac{1}{2} i f^{3 d c} V^{+\left(\frac{5}{2}\right), c}\right.\right. \\
& \left.+\frac{(2 k+7)}{6 k} i f^{3 d c} \partial G^{-, c}\right) \\
& +i f^{32 d}\left(\frac{1}{2} f^{3 d c} V^{+\left(\frac{5}{2}\right), c}+\frac{(2 k+7)}{6 k} f^{3 d c} \partial G^{-, c}\right) \\
& +i f^{31 d}\left(-\frac{1}{2} i f^{3 d c} V^{-\left(\frac{5}{2}\right), c}-\frac{7}{6 k} i f^{3 d c} \partial G^{-, c}\right) \\
& +i f^{32 d}\left(-\frac{1}{2} f^{3 d c} V^{-\left(\frac{5}{2}\right), c}-\frac{7}{6 k} f^{3 d c} \partial G^{-, c}\right) \\
& -\frac{(k+7)}{2 k} f^{31 d} f^{3 d e} G^{+, e} K \\
& +\frac{i(k+7)}{2 k} f^{32 d} f^{3 d e} G^{+, e} K
\end{aligned}
$$




$$
\begin{aligned}
& -\frac{(k+7)}{2 k} f^{31 d} f^{3 d e} G^{-, e} K-i f^{31 d} J^{d} G^{-, 3} \\
& +\frac{i(k+7)}{2 k} f^{32 d} f^{3 d e} G^{-, e} K \\
& -f^{32 d} J^{d} G^{-, 3}+i f^{31 d} G^{+, d} J_{f}^{3} \\
& +f^{32 d} G^{+, d} J_{f}^{3}+\frac{(2 k+7)}{k} \delta^{33} J^{1} G^{+} \\
& -\frac{i(2 k+7)}{k} \delta^{33} J^{2} G^{+} \\
& +i f^{31 d} G^{-, d} J_{f}^{3}+f^{32 d} G^{-, d} J_{f}^{3}+\frac{(k+7)}{k} \delta^{33} J^{1} G^{-} \\
& \left.-\frac{i(k+7)}{k} \delta^{33} J^{2} G^{-}\right] \text {, } \\
& V^{\left(\frac{5}{2}\right)}=\left(\frac{1}{k+7}\right)^{3 / 2}\left[-i f^{31 d} \frac{1}{2} f^{d e f} f^{3 f g} J^{e} G^{+, g}\right. \\
& +i f^{32 d}\left(-\frac{1}{2} f^{\text {def }} i f^{3 f g} J^{e} G^{+, g}\right. \\
& \left.-\frac{\left(k^{2}-7\right)}{2 k} f^{3 d e} \partial G^{+, e}\right) \\
& +i f^{31 d}\left(\frac{1}{2} i f^{3 d e} V^{-\left(\frac{5}{2}\right), e}-f^{d e f} f^{3 f g} J^{e} G^{-, g}\right. \\
& \left.-\frac{(k+7)}{2 k} i f^{3 d e} J^{e} G^{-}\right) \\
& +i f^{32 d}\left(-\frac{1}{2} f^{3 d e} V^{-\left(\frac{5}{2}\right), e}-i f^{d e f} f^{3 f g} J^{e} G^{-, g}\right. \\
& \left.-\frac{\left(k^{2}+10 k-14\right)}{6 k} f^{3 d e} \partial G^{-, e}+\frac{(k+7)}{2 k} f^{3 d e} J^{e} G^{-}\right) \\
& +\frac{i(k+7)}{2 k} f^{32 d} f^{3 d e} G^{+, e} K \\
& +i f^{31 d} J^{d} G^{-, 3}-f^{32 d} J^{d} G^{-, 3} \\
& -G^{+} J_{f}^{1}-2 t_{j \bar{i}}^{1} \delta_{k \bar{l}} \delta_{\rho \bar{\mu}} \delta_{\tau \bar{\sigma}} \psi^{(\bar{\sigma} j)} \psi^{(\rho \bar{i})} \psi^{(\bar{\mu} k)} J^{(\tau \bar{l})} \\
& +k \partial G^{+, 1}-2 G^{-} J_{f}^{1} \\
& -2 t_{i \bar{j}}^{1} \delta_{l \bar{k}} \delta_{\mu \bar{\rho}} \delta_{\sigma \bar{\tau}} \psi^{(\sigma \bar{j})} \psi^{(\bar{\rho} i)} \psi^{(\mu \bar{k})} J^{(\bar{\tau} l)} \\
& +\frac{\left(k^{2}+10 k-14\right)}{3 k} \partial G^{-, 1}+\sqrt{\frac{7}{5}} f^{32 c} J^{u(1)} G^{+, c} \\
& +\frac{1}{5} \sqrt{2}(5 k+1) f^{32 d} t_{j \bar{i}}^{d} \delta_{\rho \bar{\sigma}} \partial J^{(\rho \bar{i})} \psi^{(\bar{\sigma} j)} \\
& -\sqrt{2} f^{32 c} t_{\rho \bar{\sigma}}^{\alpha} t_{j \bar{i}}^{c} J^{(\rho \bar{i})} \psi^{(\bar{\sigma} j)} J^{\alpha} \\
& +i \sqrt{2} t_{l \bar{k}}^{3} \delta_{\tau \bar{\mu}}\left(\left(\psi^{(\bar{\mu} l)} J^{(\tau \bar{k})}\right) J_{f}^{2}\right) \\
& -\frac{\sqrt{\frac{7}{5}}(k+5)}{k} i f^{31 c} J^{u(1)} G^{+, c} \\
& -\frac{1}{5} \sqrt{2}(5 k+1) i f^{31 c} t_{j \bar{i}}^{c} \delta_{\rho \bar{\sigma}} \partial J^{(\rho \bar{i})} \psi^{(\bar{\sigma} j)} \\
& +\sqrt{2} i f^{31 c} t_{\rho \bar{\sigma}}^{\alpha} t_{j \bar{i}}^{c} J^{(\rho \bar{i})} \psi^{(\bar{\sigma} j)} J^{\alpha} \\
& +2 \sqrt{2} t_{\rho \bar{\sigma}}^{\alpha} t_{j \bar{i}}^{1} t_{\nu \bar{\mu}}^{\alpha} t_{k \bar{l}}^{3} \psi^{(\bar{\sigma} j)} \psi^{(\rho \bar{i})} \psi^{(\nu \bar{l})} J^{(\bar{\mu} k)} \\
& -\frac{1}{\sqrt{2}} f^{3 c e} f^{c 1 d} G^{+, e} J_{f}^{d}-\frac{\sqrt{35}}{k} f^{32 c} J^{u(1)} G^{-, c} \\
& +\frac{\sqrt{35}}{k} i f^{31 c} J^{u(1)} G^{-, c} \\
& -\frac{1}{5} \sqrt{2} i f^{31 c} t_{\bar{j} i}^{c} \delta_{\sigma \bar{\rho}} \partial J^{(\bar{\rho} i)} \psi^{(\sigma \bar{j})} \\
& \left.+\frac{12}{5} \sqrt{2} t_{\bar{l} k}^{3} \delta_{\mu \bar{\tau}}\left(\left(\psi^{(\mu \bar{l})} J^{(\bar{\tau} k)}\right) J_{f}^{1}\right)\right], \\
& W_{+}^{\left(\frac{5}{2}\right)}=\left(\frac{1}{k+7}\right)^{3 / 2}\left[\frac{(k-2)(k+1)}{2 k} f^{12 d} \delta^{3 d} \partial G^{+}\right. \\
& +\frac{1}{10}(10 k+37) f^{12 d} \delta^{3 d} \partial G^{-} \\
& -\frac{(k+7)}{7 k} f^{12 d} \delta^{3 d} G^{+} K \\
& -\frac{1}{10}(k+7) f^{12 d} \delta^{3 d} G^{-} K+i f^{b 3 c} G^{+, b} J_{f}^{c} \\
& -2 t_{j \bar{i}}^{3} \delta_{k \bar{l}} \delta_{\rho \bar{\mu}} \delta_{\tau \bar{\sigma}} \psi^{(\bar{\sigma} j)} \psi^{(\rho \bar{i})} \psi^{(\bar{\mu} k)} J^{(\tau \bar{l})} \\
& -G^{+, 3} J_{f}^{u(1)}-\frac{\left(k^{2}+13 k+7\right)}{3 k} \partial G^{+, 3} \\
& +2 i f^{b 3 c} G^{-, b} J_{f}^{c}-2 \delta_{l \bar{k}} \delta_{\mu \bar{\rho}} \delta_{\sigma \bar{\tau}} t_{\bar{i} j}^{3} \psi^{(\sigma \bar{j})} \psi^{(\bar{\rho} i)} \\
& \psi^{(\mu \bar{k})} J^{(\bar{\tau} l)}+G^{-, 3} J_{f}^{u(1)} \\
& -\frac{\left(2 k^{2}+8 k-7\right)}{3 k} \partial G^{-, 3} \\
& +\frac{1}{70} \sqrt{2}(35 k+2) \delta^{33} \delta_{\rho \bar{\sigma}} \delta_{j \bar{i}} \partial J^{(\rho \bar{i})} \psi^{(\bar{\sigma}) j} \\
& -\frac{10}{7} \sqrt{2} t_{\rho \bar{\sigma}}^{\alpha} t_{j \bar{i}}^{3} t_{\mu \bar{\nu}}^{\alpha} t_{l \bar{k}}^{3} \psi^{(\bar{\sigma} j)} \psi^{(\rho \bar{i})} \psi^{(\bar{v} l)} J^{(\mu \bar{k})} \\
& -\frac{(7 k+10)}{\sqrt{35} k} \delta^{33} J^{u(1)} G^{+} \\
& -\sqrt{2} \delta^{33} \delta_{j \bar{i}} t_{\rho \bar{\sigma}}^{\alpha} J^{(\rho \bar{i})} \psi^{(\bar{\sigma} j)} J^{\alpha} \\
& +\frac{1}{35} \sqrt{2}(35 k+12) \delta^{33} \delta_{i \bar{j}} \delta_{\sigma \bar{\rho}} \partial J^{(\bar{\rho} i)} \psi^{(\sigma \bar{j})} \\
& +\sqrt{2} \delta^{33} \delta_{i \bar{j}} t_{\sigma \bar{\rho}}^{\alpha} J^{(\bar{\rho} i)} \psi^{(\sigma \bar{j})} J^{\alpha} \\
& +\frac{10}{7} \sqrt{2} t_{\sigma \bar{\rho}}^{\alpha} t_{i \bar{j}}^{3} t_{\nu \bar{\mu}}^{\alpha} t_{k \bar{l}}^{3} \psi^{(\sigma \bar{j})} \psi^{(\bar{\rho} i)} \psi^{(\nu \bar{l})} J^{(\bar{\mu} k)} \\
& +\frac{(7 k+60)}{35 \sqrt{2}} \delta^{33} G^{-} J_{f}^{u(1)} \\
& -\frac{1}{\sqrt{2}} f^{3 c d} f^{3 d e} J^{c} G^{+, e}+\sqrt{2} J^{3} G^{+, 3}+\sqrt{2} J^{3} G^{-, 3} \\
& -V^{+\left(\frac{5}{2}\right), 3}+V^{-\left(\frac{5}{2}\right), 3}-\frac{(k+7)}{k} G^{+, 3} K \\
& -\frac{(2 k+7)}{k} J^{3} G^{+} \\
& -\frac{(k+7)}{k} G^{-, 3} K-\frac{7}{k} J^{3} G^{-}-i \sqrt{2} J^{1} G^{-, 2} \\
& +\frac{5}{7} f^{31 d} G^{+, d} J_{f}^{2} \\
& +\frac{12}{7} f^{31 d} G^{-, d} J_{f}^{2}+\frac{5}{14} f^{e 1 c} f^{2 c d} f^{3 e f} G^{+, f} J_{f}^{d} \\
& +\frac{6}{7} f^{e 1 c} f^{2 c d} f^{3 e f} G^{-, f} J_{f}^{d} \\
& \left.-\frac{i}{\sqrt{2}} f^{e 1 c} f^{2 c d} J^{e} G^{-, d}\right] \\
& W_{-}^{\left(\frac{5}{2}\right)}=\left(\frac{1}{k+7}\right)^{3 / 2}\left[\frac{\left(k^{2}+3 k-6\right)}{k} f^{12 d} \delta^{3 d} \partial G^{+}\right. \\
& +\frac{1}{10}(5 k+2) f^{12 d} \delta^{3 d} \partial G^{-} \\
& -\frac{6(k+7)}{7 k} f^{12 d} \delta^{3 d} G^{+} K-\frac{1}{10}(k+7) f^{12 d} \delta^{3 d} G^{-} K \\
& +2 i f^{b 3 c} G^{+, b} J_{f}^{c} \\
& -2 t_{j \bar{i}}^{3} \delta_{k \bar{l}} \delta_{\rho \bar{\mu}} \delta_{\tau \bar{\sigma}} \psi^{(\bar{\sigma} j)} \psi^{(\rho \bar{i})} \psi^{(\bar{\mu} k)} J^{(\tau \bar{l})}-G^{+, 3} J_{f}^{u(1)} \\
& -\frac{\left(2 k^{2}+8 k-7\right)}{3 k} \partial G^{+, 3}
\end{aligned}
$$




$$
\begin{aligned}
& +i f^{b 3 c} G^{-, b} J_{f}^{c}-2 \delta_{l \bar{k}} \delta_{\mu \bar{\rho}} \delta_{\sigma \bar{\tau}} t_{i \bar{j}}^{3} \psi^{(\sigma \bar{j})} \psi^{(\bar{\rho} i)} \psi^{(\mu \bar{k})} J^{(\bar{\tau} l)} \\
& +G^{-, 3} J_{f}^{u(1)} \\
& -\frac{\left(k^{2}+13 k+7\right)}{3 k} \partial G^{-, 3} \\
& +\frac{1}{35} \sqrt{2}(35 k+12) \delta^{33} \delta_{\rho \bar{\sigma}} \delta_{j \bar{i}} \partial J^{(\rho \bar{i})} \psi^{(\bar{\sigma}) j} \\
& +\frac{10}{7} \sqrt{2} t_{\rho \bar{\sigma}}^{\alpha} t_{j \bar{i}}^{3} t_{\mu \bar{\nu}}^{\alpha} t_{l \bar{k}}^{3} \psi^{(\bar{\sigma} j)} \psi^{(\rho \bar{i})} \psi^{(\bar{v} l)} J^{(\mu \bar{k})} \\
& -\frac{(7 k+60)}{\sqrt{35} k} \delta^{33} J^{u(1)} G^{+} \\
& -\sqrt{2} \delta^{33} \delta_{j \bar{i}} t_{\rho \bar{\sigma}}^{\alpha} J^{(\rho \bar{i})} \psi^{(\bar{\sigma} j)} J^{\alpha} \\
& +\frac{1}{35} \sqrt{2}(35 k+2) \delta^{33} \delta_{i \bar{j}} \delta_{\sigma \bar{\rho}} \partial J^{(\bar{\rho} i)} \psi^{(\sigma \bar{j})} \\
& +\sqrt{2} \delta^{33} \delta_{i \bar{j}} t_{\sigma \bar{\rho}}^{\alpha} J^{(\bar{\rho} i)} \psi^{(\sigma \bar{j})} J^{\alpha} \\
& -\frac{10}{7} \sqrt{2} t_{\sigma \bar{\rho}}^{\alpha} t_{i \bar{j}}^{3} t_{v \bar{\mu}}^{\alpha} t_{k \bar{l}}^{3} \psi^{(\sigma \bar{j})} \psi^{(\bar{\rho} i)} \psi^{(\nu \bar{l})} J^{(\bar{\mu} k)} \\
& +\frac{(7 k+10)}{35 \sqrt{2}} \delta^{33} G^{-} J_{f}^{u(1)} \\
& -\sqrt{2} J^{3} G^{+, 3}+\frac{1}{\sqrt{2}} f^{3 c d} f^{3 d e} J^{c} G^{-, e} \\
& -\sqrt{2} J^{3} G^{-, 3}+V^{+\left(\frac{5}{2}\right), 3}-V^{-\left(\frac{5}{2}\right), 3}+\frac{(k+7)}{k} G^{+, 3} K \\
& +\frac{7}{k} J^{3} G^{+}+\frac{(k+7)}{k} G^{-, 3} K+\frac{(2 k+7)}{k} J^{3} G^{-} \\
& -i \sqrt{2} J^{1} G^{+, 2}-\frac{12}{7} f^{31 d} G^{+, d} J_{f}^{2}-\frac{5}{7} f^{31 d} G^{-, d} J_{f}^{2} \\
& -\frac{6}{7} f^{e 1 c} f^{2 c d} f^{3 e f} G^{+, f} J_{f}^{d} \\
& -\frac{5}{14} f^{e 1 c} f^{2 c d} f^{3 e f} G^{-, f} J_{f}^{d} \\
& \left.-\frac{i}{\sqrt{2}} f^{e 1 c} f^{2 c d} J^{e} G^{+, d}\right] \text {. }
\end{aligned}
$$

The general $N$ dependence on these currents can be determined by calculating Appendix (G.4) explicitly. Then the spin- $\frac{5}{2}$ currents can be obtained from $V^{ \pm\left(\frac{5}{2}\right), a}$ and other composite operators.

\section{References}

1. T. Creutzig, Y. Hikida, P.B. Ronne, Extended higher spin holography and Grassmannian models. JHEP 11, 038 (2013). https://doi. org/10.1007/JHEP11(2013)038. arXiv:1306.0466 [hep-th]

2. L. Eberhardt, T. Procházka, The Grassmannian VOA. JHEP 09, 150 (2020). https://doi.org/10.1007/JHEP09(2020)150. arXiv:2006.02422 [hep-th]

3. T. Creutzig, Y. Hikida, Rectangular W-algebras, extended higher spin gravity and dual coset CFTs. JHEP 02, 147 (2019). https:// doi.org/10.1007/JHEP02(2019)147. arXiv:1812.07149 [hep-th]

4. S.F. Prokushkin, M.A. Vasiliev, Higher spin gauge interactions for massive matter fields in 3-D AdS space-time. Nucl. Phys. B 545, 385 (1999). https://doi.org/10.1016/ S0550-3213(98)00839-6. arXiv:hep-th/9806236

5. S. Prokushkin, M.A. Vasiliev, 3-d higher spin gauge theories with matter. arXiv:hep-th/9812242

6. M.R. Gaberdiel, R. Gopakumar, $\mathrm{An}_{\mathrm{AdS}} 3$ dual for minimal model CFTs. Phys. Rev. D 83, 066007 (2011). https://doi.org/10.1103/ PhysRevD.83.066007. arXiv:1011.2986 [hep-th]
7. M.R. Gaberdiel, R. Gopakumar, Triality in minimal model holography. JHEP 07, 127 (2012). https://doi.org/10.1007/ JHEP07(2012)127. arXiv:1205.2472 [hep-th]

8. M.R. Gaberdiel, R. Gopakumar, Minimal model holography. J. Phys. A 46, 214002 (2013). https://doi.org/10.1088/1751-8113/ 46/21/214002. arXiv:1207.6697 [hep-th]

9. M. Ammon, M. Gutperle, P. Kraus, E. Perlmutter, Black holes in three dimensional higher spin gravity: a review. J. Phys. A 46, 214001 (2013). https://doi.org/10.1088/1751-8113/46/21/ 214001. arXiv:1208.5182 [hep-th]

10. T. Arakawa, A. Molev, Explicit generators in rectangular affine $\mathcal{W}$ algebras of type A. Lett. Math. Phys. 107(1), 47-59 (2017). https:// doi.org/10.1007/s11005-016-0890-2. arXiv:1403.1017 [math.RT]

11. L. Eberhardt, T. Procházka, The matrix-extended $W_{1+\infty}$ algebra. JHEP 12, 175 (2019). https://doi.org/10.1007/JHEP12(2019)175. arXiv: 1910.00041 [hep-th]

12. C. Ahn, The Grassmannian-like coset model and the higher spin currents. JHEP 03, 037 (2021). https://doi.org/10.1007/ JHEP03(2021)037. arXiv:2011.11240 [hep-th]

13. C. Ahn, The coset spin- 4 casimir operator and its three-point functions with scalars. JHEP 02, 027 (2012). https://doi.org/10.1007/ JHEP02(2012)027. arXiv:1111.0091 [hep-th]

14. C. Ahn, H. Kim, Spin-5 casimir operator its three-point functions with two scalars. JHEP 01, 012 (2014). https://doi.org/10.1007/ JHEP01(2014)012. arXiv:1308.1726 [hep-th] (Erratum: JHEP 01, 174 (2014))

15. I. Bakas, E. Kiritsis, Grassmannian coset models and unitary representations of $W$ (infinity). Mod. Phys. Lett. A 5, 2039-2050 (1990). https://doi.org/10.1142/S0217732390002328

16. M.R. Douglas, "G / H Conformal Field Theory," CALT-68-1453

17. F.A. Bais, P. Bouwknegt, M. Surridge, K. Schoutens, "Extended Virasoro Algebras," Copenhagen String Th.1987:0023

18. P. Goddard, A. Schwimmer, Unitary construction of extended conformal algebras. Phys. Lett. B 206, 62 (1988). https://doi.org/10. 1016/0370-2693(88)91263-4

19. P. Goddard, A. Kent, D.I. Olive, Unitary representations of the Virasoro and Supervirasoro algebras. Commun. Math. Phys. 103, 105 (1986). https://doi.org/10.1007/BF01464283

20. K. Hornfeck, E. Ragoucy, A coset construction for the super $W(3)$ algebra. Nucl. Phys. B 340, 225 (1990). https://doi.org/10.1016/ 0550-3213(90)90162-7

21. C. Ahn, K. Schoutens, A. Sevrin, The full structure of the super W(3) algebra. Int. J. Mod. Phys. A 6, 3467 (1991). https://doi.org/ 10.1142/S0217751X91001684

22. K. Schoutens, A. Sevrin, Minimal superW(N) algebras in coset conformal field theories. Phys. Lett. B 258, 134 (1991). https://doi. org/10.1016/0370-2693(91)91220-P

23. C. Ahn, The higher spin currents in the $\mathrm{N}=1$ stringy coset minimal model. JHEP 1304, 033 (2013). https://doi.org/10.1007/ JHEP04(2013)033. arXiv:1211.2589 [hep-th]

24. C. Ahn, Higher spin currents with arbitrary $N$ in the $\mathcal{N}=1$ stringy coset minimal model. JHEP 1307, 141 (2013). https://doi.org/10. 1007/JHEP07(2013)141. arXiv:1305.5892 [hep-th]

25. W. Boucher, D. Friedan, A. Kent, Determinant formulae and unitarity for the $\mathrm{N}=2$ superconformal algebras in two-dimensions or exact results on string compactification. Phys. Lett. B 172, 316 (1986). https://doi.org/10.1016/0370-2693(86)90260-1

26. R. Gopakumar, A. Hashimoto, I.R. Klebanov, S. Sachdev, K. Schoutens, Strange metals in one spatial dimension. Phys. Rev. D 86, 066003 (2012). https://doi.org/10.1103/PhysRevD.86.066003. arXiv: 1206.4719 [hep-th]

27. C. Ahn, Higher spin currents in the $\mathrm{N}=2$ stringy coset minimal model. Phys. Rev. D 94(12), 126014 (2016). https://doi.org/10. 1103/PhysRevD.94.126014. arXiv:1604.00756 [hep-th] 
28. C. Ahn, Higher spin currents in the orthogonal coset theory. Eur. Phys. J. C 77(6), 394 (2017). https://doi.org/10.1140/epjc/ s10052-017-4956-9. arXiv:1701.02410 [hep-th]

29. C. Ahn, J. Paeng, A supersymmetric enhancement of $\mathcal{N}=1$ holographic minimal model. JHEP 05, 135 (2019). https://doi.org/10. 1007/JHEP05(2019)135. arXiv:1902.03699 [hep-th]

30. T. Creutzig, Y. Hikida, Rectangular W algebras and superalgebras and their representations. Phys. Rev. D 100(8), 086008 (2019). https://doi.org/10.1103/PhysRevD.100.086008. arXiv:1906.05868 [hep-th]

31. Y. Kazama, H. Suzuki, New N=2 superconformal field theories and superstring compactification. Nucl. Phys. B 321, 232-268 (1989). https://doi.org/10.1016/0550-3213(89)90250-2

32. Y. Kazama, H. Suzuki, Characterization of $\mathrm{N}=2$ superconformal models generated by coset space method. Phys. Lett. B 216, 112116 (1989). https://doi.org/10.1016/0370-2693(89)91378-6

33. T. Creutzig, Y. Hikida, P.B. Ronne, Higher spin $\mathrm{AdS}_{3}$ supergravity and its dual CFT. JHEP 02, 109 (2012). https://doi.org/10.1007/ JHEP02(2012)109. arXiv:1111.2139 [hep-th]

34. C. Candu, M.R. Gaberdiel, Supersymmetric holography on $A d S_{3}$. JHEP 09, 071 (2013). https://doi.org/10.1007/JHEP09(2013)071. arXiv:1203.1939 [hep-th]

35. M. Henneaux, G. Lucena Gómez, J. Park, S.J. Rey, SuperW(infinity) asymptotic symmetry of higher-spin $A d S_{3}$ supergravity. JHEP 06, 037 (2012). https://doi.org/10.1007/ JHEP06(2012)037. arXiv:1203.5152 [hep-th]

36. K. Hanaki, C. Peng, Symmetries of holographic superminimal models. JHEP 08, 030 (2013). https://doi.org/10.1007/ JHEP08(2013)030. arXiv:1203.5768 [hep-th]

37. C. Ahn, The large N 't Hooft Limit of Kazama-Suzuki model. JHEP 08, 047 (2012). https://doi.org/10.1007/JHEP08(2012)047. arXiv:1206.0054 [hep-th]

38. C. Candu, M.R. Gaberdiel, Duality in $\mathrm{N}=2$ minimal model holography. JHEP 02, 070 (2013). https://doi.org/10.1007/ JHEP02(2013)070. arXiv:1207.6646 [hep-th]

39. C. Ahn, The operator product expansion of the lowest higher spin current at finite N. JHEP 01, 041 (2013). https://doi.org/10.1007/ JHEP01(2013)041. arXiv:1208.0058 [hep-th]

40. T. Creutzig, Y. Hikida, P.B. Ronne, Three point functions in higher spin $\mathrm{AdS}_{3}$ supergravity. JHEP 01, 171 (2013). https://doi.org/10. 1007/JHEP01(2013)171. arXiv:1211.2237 [hep-th]

41. M.R. Gaberdiel, M. Kelm, The continuous orbifold of $\mathcal{N}=2$ minimal model holography. JHEP 08, 084 (2014). https://doi.org/ 10.1007/JHEP08(2014)084. arXiv:1406.2345 [hep-th]

42. M.R. Gaberdiel, M. Kelm, The symmetric orbifold of $\mathcal{N}=2$ minimal models. JHEP 07, 113 (2016). https://doi.org/10.1007/ JHEP07(2016)113. arXiv:1604.03964 [hep-th]

43. S. Datta, L. Eberhardt, M.R. Gaberdiel, Stringy $\mathcal{N}=(2,2)$ holography for $\mathrm{AdS}_{3}$. JHEP 01, 146 (2018). https://doi.org/10.1007/ JHEP01(2018)146. arXiv:1709.06393 [hep-th]

44. L. Eberhardt, M.R. Gaberdiel, I. Rienacker, Higher spin algebras and large $\mathcal{N}=4$ holography. JHEP 03, 097 (2018). https://doi.org/ 10.1007/JHEP03(2018)097. arXiv:1801.00806 [hep-th]

45. A. Castro, A. Faraggi, I. Osorio, A note on the $\mathcal{N}=2$ super- $\mathcal{W}_{3}$ holographic dictionary. JHEP 12, 177 (2020). https://doi.org/10. 1007/JHEP12(2020)177. arXiv:2008.05541 [hep-th]

46. M.R. Gaberdiel, R. Gopakumar, Large $N=4$ holography. JHEP 09, 036 (2013). https://doi.org/10.1007/JHEP09(2013)036. arXiv:1305.4181 [hep-th]

47. C. Ahn, Higher spin currents in wolf space. Part I. JHEP 03, 091 (2014). https://doi.org/10.1007/JHEP03(2014)091. arXiv:1311.6205 [hep-th]

48. M.R. Gaberdiel, C. Peng, The symmetry of large $\mathcal{N}=$ 4 holography. JHEP 05, 152 (2014). https://doi.org/10.1007/ JHEP05(2014)152. arXiv:1403.2396 [hep-th]
49. M. Beccaria, C. Candu, M.R. Gaberdiel, The large $\mathrm{N}=4$ superconformal $W_{\infty}$ algebra. JHEP 06, 117 (2014). https://doi.org/10. 1007/JHEP06(2014)117. arXiv:1404.1694 [hep-th]

50. M.R. Gaberdiel, R. Gopakumar, Higher spins and strings. JHEP 11, 044 (2014). https://doi.org/10.1007/JHEP11(2014)044. arXiv:1406.6103 [hep-th]

51. C. Ahn, Higher spin currents in wolf space: part II. Class. Quantum Gravity 32(1), 015023 (2015). https://doi.org/10.1088/0264-9381/ 32/1/015023. arXiv:1408.0655 [hep-th]

52. C. Ahn, J. Paeng, Higher spin currents in orthogonal wolf space. Class. Quantum Gravity 32(4), 045011 (2015). https://doi.org/10. 1088/0264-9381/32/4/045011. arXiv:1410.0080 [hep-th]

53. M.R. Gaberdiel, R. Gopakumar, Stringy symmetries and the higher spin square. J. Phys. A 48(18), 185402 (2015). https://doi.org/10. 1088/1751-8113/48/18/185402. arXiv:1501.07236 [hep-th]

54. C. Ahn, Higher spin currents in Wolf space: III. Class. Quantum Gravity 32(18), 185001 (2015). https://doi.org/10.1088/ 0264-9381/32/18/185001. arXiv:1504.00070 [hep-th]

55. C. Ahn, H. Kim, Three point functions in the large $\mathcal{N}=$ 4 holography. JHEP 10, 111 (2015). https://doi.org/10.1007/ JHEP10(2015)111. arXiv:1506.00357 [hep-th]

56. C. Ahn, M.H. Kim, The operator product expansion between the 16 lowest higher spin currents in the $\mathcal{N}=4$ superspace. Eur. Phys. J. C 76(7), 389 (2016). https://doi.org/10.1140/epjc/ s10052-016-4234-2. arXiv:1509.01908 [hep-th]

57. C. Ahn, H. Kim, J. Paeng, Three-point functions in the $\mathscr{N}$ $=4$ orthogonal coset theory. Int. J. Mod. Phys. A 31(16), 1650090 (2016). https://doi.org/10.1142/S0217751X16500901. arXiv:1510.03139 [hep-th]

58. M.R. Gaberdiel, R. Gopakumar, String theory as a higher spin theory. JHEP 09, 085 (2016). https://doi.org/10.1007/ JHEP09(2016)085. arXiv:1512.07237 [hep-th]

59. C. Ahn, H. Kim, Higher spin currents in the enhanced $\mathcal{N}=3$ Kazama-Suzuki model. JHEP 12, 001 (2016). https://doi.org/10. 1007/JHEP12(2016)001. arXiv:1607.00728 [hep-th]

60. C. Ahn, J. Hong, M.H. Kim, Fermionic construction in the supersymmetric coset model. arXiv:2106.03342 [hep-th]

61. K. Ferreira, Even spin $\mathcal{N}=4$ holography. JHEP 09, 110 (2017). https://doi.org/10.1007/JHEP09(2017)110. arXiv:1702.02641 [hep-th]

62. C. Ahn, D.G. Kim, M.H. Kim, The next 16 higher spin currents and three-point functions in the large $\mathcal{N}=4$ holography. Eur. Phys. J. C 77(8), 523 (2017). https://doi.org/10.1140/epjc/ s10052-017-5064-6. arXiv:1703.01744 [hep-th]

63. L. Eberhardt, M.R. Gaberdiel, W. Li, A holographic dual for string theory on $\mathrm{AdS}_{3} \times \mathrm{S}^{3} \times \mathrm{S}^{3} \times \mathrm{S}^{1}$. JHEP 08, 111 (2017). https://doi. org/10.1007/JHEP08(2017)111. arXiv:1707.02705 [hep-th]

64. C. Ahn, Wolf space coset spectrum in the large $\mathcal{N}=4$ holography. J. Phys. A 51(43), 435402 (2018). https://doi.org/10.1088/ 1751-8121/aae15d. arXiv:1711.07599 [hep-th]

65. C. Ahn, Higher spin currents with manifest $S O(4)$ symmetry in the large $\mathcal{N}=4$ holography. Int. J. Mod. Phys. A 33(35), 1850208 (2018). https://doi.org/10.1142/S0217751X18502081. arXiv: 1805.02298 [hep-th]

66. L. Eberhardt, M.R. Gaberdiel, Strings on $A d S_{3} \times S^{3} \times S^{3} \times S^{1}$. JHEP 06, 035 (2019). https://doi.org/10.1007/JHEP06(2019)035. arXiv:1904.01585 [hep-th]

67. C. Ahn, M.H. Kim, J. Paeng, The operator product expansions in the $\mathcal{N}=4$ orthogonal Wolf space coset model. Eur. Phys. J. C 79(7), 578 (2019). https://doi.org/10.1140/epjc/ s10052-019-7091-y. arXiv:1904.06855 [hep-th]

68. C. Ahn, Dg. Kim, M.H. Kim, The $\mathcal{N}=4$ coset model and the higher spin algebra. Int. J. Mod. Phys. A 35(11n12), 2050046 (2020). https://doi.org/10.1142/S0217751X20500463. arXiv:1910.02183 [hep-th] 
69. C. Ahn, M.R. Gaberdiel, M.H. Kim, The small $\mathcal{N}=4$ superconformal $\mathcal{W}_{\infty}$ algebra. J. Phys. A 53(39), 395401 (2020). https://doi. org/10.1088/1751-8121/aba5ba. arXiv:2004.07439 [hep-th]

70. C. Ahn, M.H. Kim, The $\mathcal{N}=4$ higher spin algebra for generic $\mu$ parameter. JHEP 02, 123 (2021). https://doi.org/10.1007/ JHEP02(2021)123. arXiv:2009.04852 [hep-th]

71. M.R. Gaberdiel, R. Gopakumar, The worldsheet dual of free super Yang-Mills in 4D. arXiv:2105.10496 [hep-th]

72. C. Ahn, H. Kim, Higher spin currents in wolf space for generic N. JHEP 12, 109 (2014). https://doi.org/10.1007/JHEP12(2014)109. arXiv:1411.0356 [hep-th]

73. A. Van Proeyen, Realizations of $\mathrm{N}=4$ superconformal algebras on wolf spaces. Class. Quantum Gravity 6, 1501 (1989)

74. P. Goddard, A. Schwimmer, Factoring out free fermions and superconformal algebras. Phys. Lett. B 214, 209 (1988)

75. M. Gunaydin, J.L. Petersen, A. Taormina, A. Van Proeyen, On the unitary representations of a class of $\mathrm{N}=4$ superconformal algebras. Nucl. Phys. B 322, 402 (1989)

76. S.J. Gates Jr., S.V. Ketov, No N=4 strings on wolf spaces. Phys. Rev. D 52, 2278 (1995). arXiv:hep-th/9501140

77. F.A. Bais, P. Bouwknegt, M. Surridge, K. Schoutens, Coset construction for extended Virasoro algebras. Nucl. Phys. B 304, 371391 (1988). https://doi.org/10.1016/0550-3213(88)90632-3

78. P. Bouwknegt, K. Schoutens, W symmetry in conformal field theory. Phys. Rep. 223, 183 (1993). https://doi.org/10.1016/ 0370-1573(93)90111-P. arXiv:hep-th/9210010

79. F.A. Bais, P. Bouwknegt, M. Surridge, K. Schoutens, Extensions of the Virasoro algebra constructed from Kac-Moody algebras using higher order casimir invariants. Nucl. Phys. B 304, 348-370 (1988). https://doi.org/10.1016/0550-3213(88)90631-1

80. K. Thielemans, A mathematica package for computing operator product expansions. Int. J. Mod. Phys. C 2, 787 (1991). https://doi. org/10.1142/S0129183191001001

81. Wolfram Research, Inc., Mathematica, Version 12.1, Champaign, IL (2020)

82. T. Creutzig, Y. Hikida, P.B. Ronne, Higher spin $\mathrm{AdS}_{3}$ holography with extended supersymmetry. JHEP 1410, 163 (2014). https://doi. org/10.1007/JHEP10(2014)163. arXiv:1406.1521 [hep-th]

83. C. Ahn, The large N 't Hooft limit of coset minimal models. JHEP 10, 125 (2011). https://doi.org/10.1007/JHEP10(2011)125. arXiv:1106.0351 [hep-th]
84. C. Ahn, The primary spin- 4 casimir operators in the holographic SO(N) coset minimal models. JHEP 05, 040 (2012). https://doi. org/10.1007/JHEP05(2012)040. arXiv:1202.0074 [hep-th]

85. C. Ahn, J. Paeng, The OPEs of spin- 4 casimir currents in the holographic $S O(N)$ coset minimal models. Class. Quantum Gravity 30, 175004 (2013). https://doi.org/10.1088/0264-9381/30/17/ 175004. arXiv:1301.0208 [hep-th]

86. C. Ahn, J. Paeng, Higher spin currents in the holographic $\mathcal{N}=$ 1 coset minimal model. JHEP 01, 007 (2014). https://doi.org/10. 1007/JHEP01(2014)007. arXiv:1310.6185 [hep-th]

87. T. Creutzig, Y. Hikida, T. Uetoko, Rectangular W-algebras of types $s o(M)$ and $s p(2 M)$ and dual coset CFTs. JHEP 10, 023 (2019). https://doi.org/10.1007/JHEP10(2019)023. arXiv:1906.05872 [hep-th]

88. A. Sevrin, W. Troost, A. Van Proeyen, Superconformal algebras in two-dimensions with $\mathrm{N}=4$. Phys. Lett. B 208, 447 (1988)

89. A. Sevrin, W. Troost, A. Van Proeyen, P. Spindel, Extended supersymmetric sigma models on group manifolds. 2. Current algebras. Nucl. Phys. B 311, 465 (1988)

90. K. Schoutens, O(n) extended superconformal field theory in superspace. Nucl. Phys. B 295, 634 (1988)

91. E.A. Ivanov, S.O. Krivonos, $\mathrm{N}=4$ superliouville equation (in Russian). J. Phys. A 17, L671 (1984)

92. E.A. Ivanov, S.O. Krivonos, $N=4$ Superextension of the Liouville equation with quaternionic structure. Theor. Math. Phys. 63, 477 (1985) (Teor. Mat. Fiz. 63, 230 (1985))

93. E.A. Ivanov, S.O. Krivonos, V.M. Leviant, A new class of superconformal $\sigma$ models with the Wess-Zumino action. Nucl. Phys. B 304, 601 (1988)

94. E.A. Ivanov, S.O. Krivonos, V.M. Leviant, Quantum $\mathrm{N}=3, \mathrm{~N}=$ 4 Superconformal WZW sigma models. Phys. Lett. B 215, 689 (1988) (Erratum-ibid. B 221, 432 (1989))

95. A. Sevrin, G. Theodoridis, $\mathrm{N}=4$ superconformal coset theories. Nucl. Phys. B 332, 380 (1990)

96. N. Saulina, Geometric interpretation of the large $\mathrm{N}=4$ index. Nucl. Phys. B 706, 491 (2005). arXiv:hep-th/0409175 